\title{
OPTIMAL EXPERIMENTAL DESIGN UNDER
}

\section{A NEW MULTIVARIATE WEIBULL REGRESSION FUNCTION}

A Dissertation presented to

the Faculty of the Graduate School

at the University of Missouri

In Partial Fulfillment

of the Requirements for the Degree

Doctor of Philosophy

by

SUNGWOOK KIM

Dr. Nancy Flournoy, Dissertation Supervisor

MAY 2014 
The undersigned, appointed by the Dean of the Graduate School, have examined the dissertation entitled:

OPTIMAL EXPERIMENTAL DESIGN UNDER

\section{A NEW MULTIVARIATE WEIBULL REGRESSION FUNCTION}

presented by Sungwook Kim, a candidate for the degree of Doctor of Philosophy and hereby certify that, in their opinion, it is worthy of acceptance.

Dr. Nancy Flournoy

Dr. Zhuoqiong He

Dr. Toni Kazic

Dr. Jianguo Sun

Dr. Lori Thombs 


\section{ACKNOWLEDGMENTS}

I would like to express the deepest appreciation to my advisor Dr. Nancy Flournoy. Without her guidance and persistent help this dissertation would not have been pos-

sible. I really deeply appreciate her supervising and support. I will never forget my Ph.D life with her in the University of Missouri and Cambridge. I extend my thanks to my committee members: Dr. Zhuoqiong He, Dr. Toni Kazic, Dr. Jianguo Sun, and Dr. Lori Thombs for their time, insightful comments and suggestions on this dissertation.

In addition, I thank Dr. Leonard Hearne, who encouraged me when I struggled for the purpose of this study and my future plans. I will never forget his mentoring. Foremost, I thank God who loves me and my wife Bokyung Kim whom I love. Thank you all of the faculty members, staffs and colleagues in the Department of Statistics. 


\section{TABLE OF CONTENTS}

ACKNOWLEDGMENTS ................ ii

LIST OF TABLES $\ldots \ldots \ldots \ldots \ldots \ldots \ldots$ vii

LIST OF FIGURES $\ldots \ldots \ldots \ldots \ldots \ldots$ viii

ABSTRACT ....................

\section{CHAPTER}

1 Introduction ...................... 1

2 Background Theory $\ldots \ldots \ldots \ldots \ldots \ldots \ldots$

2.1 The Design Theory $\ldots \ldots \ldots \ldots \ldots \ldots \ldots$

2.2 Optimality Criteria (A Review) $\ldots \ldots \ldots \ldots \ldots$

$2.2 .1 \quad D$-optimality (Determinant) $\ldots \ldots \ldots \ldots$

2.2.2 $D_{A^{-} \text {optimality }(\text { Generalized D-optimality) } \ldots \ldots \ldots} \ldots$

$2.2 .3 \quad$ A-optimality (Average or Trace) . . . . . . . . . . . . 10

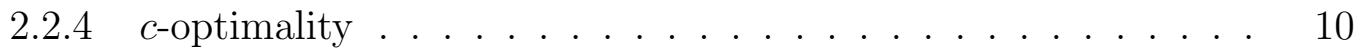

$2.2 .5 \quad$ E-optimality (Eigenvalue) . . . . . . . . . . . 10

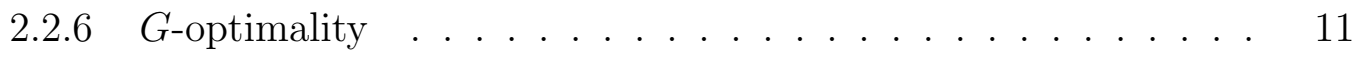

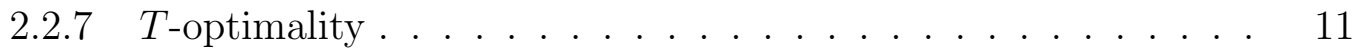

$2.2 .8 \quad I$-optimality (Integrated) $\ldots \ldots \ldots \ldots \ldots \ldots$

$2.2 .9 \quad V$-optimality (Variance) $\ldots \ldots \ldots \ldots \ldots$

2.2.10 The General Equivalence Theorem . . . . . . . . . . . 12 
2.2 .11 Carathéodory's Theorem . . . . . . . . . . . . . . . . . 12

3 Methods for Constructing Bivariate Density Functions . . . . . . . 14

3.1 The Basic Idea $\ldots \ldots \ldots \ldots$

3.1 .1 Frechet Bounds . . . . . . . . . . . . . . . . . . 14

3.1 .2 Transformations. . . . . . . . . . . . . . . 15

3.2 Product of a Conditional Density and a Marginal Density . . . . . . 15

3.3 The Monotone Transformation Method . . . . . . . . . . . . 16

3.4 Methods for Constructing Copulas . . . . . . . . . . . . 17

3.4.1 The Univariate Function Method . . . . . . . . . . . . 17

3.4.2 The Inversion Method (Marginal Transformation Method) . . 18

3.4 .3 Geometric Methods . . . . . . . . . . . . . . . . . . . . 18

3.4 .4 Algebraic Methods . . . . . . . . . . . . . . . 18

3.4.5 The Rüschendorf's Method . . . . . . . . . . . . . . 19

3.4.6 Constructing a Copula from a Distortion Function . . . . . . . 19

3.5 Uniform Representation . . . . . . . . . . . . . . . 20

3.6 Marshall and Olkin's Mixture Method _... . . . . . . . . . 21

4 A New Bivariate Weibull Function . . . . . . . . . . . . . 22

4.1 A New Bivariate Weibull Regression Model . . . . . . . . . . . . 22

4.1.1 A Bivariate Weibull Density . . . . . . . . . . . . . 22

4.1.2 A New Bivariate Weibull Regression Model . . . . . . . . . 25

4.2 Optimal Experimental Designs with Bivariate Outcomes . . . . . 26

4.2.1 Two Dependent Binary Outcomes . . . . . . . . . . . . . 26 
4.2 .2 A Locally Optimal Design . . . . . . . . . . . . . . . . . 29

4.2 .3 A Penalized Locally Optimal Design _. . . . . . . . . . 30

4.2.4 Information Matrix . . . . . . . . . . . . . . 33

4.2.5 The Equivalence Theorem . . . . . . . . . . . . . 35

4.3 A Crash Test with Two Components: An Example . . . . . . . . 36

4.4 Robustness . . . . . . . . . . . . . . . . . . . . 48

4.5 An Adaptive Optimal Design _ . . . . . . . . . . . . . . 52

5 A Trivariate Serial System subjected to Decreasing Damage . . . 56

5.1 A Trivariate System with Components having Decreasing Damage . . 56

5.1 .1 Motivation . . . . . . . . . . . . . 56

5.1 .2 A Trivariate Weibull Model . . . . . . . . . . . . . . . 60

5.1.3 A Trivariate Weibull Regression Model . . . . . . . . . . . . 64

5.2 Information Matrix and a Locally Optimal Design . . . . . . . . . 64

5.3 c-Optimal Designs for an Effective Stress . . . . . . . . . . 67

5.4 An Adaptive Optimal Design for Implementation . . . . . . . . . 71

6 Future Studies . . . . . . . . . . . . . . . 74

6.1 Multivariate Weibull Probability Density Function . . . . . . . . . 74

6.2 Optimal Designs for Serial and Parallel Systems with Two Binary Out-

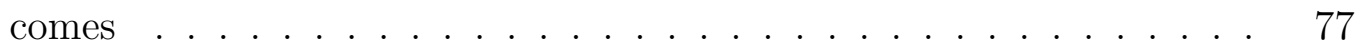

\section{APPENDIX}

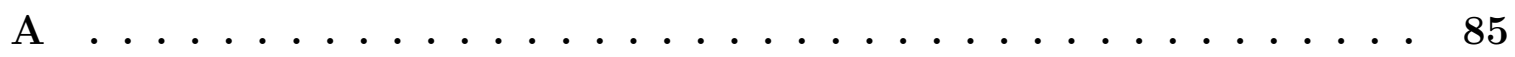

A.1 Outcome Probabilities . . . . . . . . . . . . . . 85

A.2 Outcome Probabilities with Predictor Functions (Equation (4.10)) . 87 
A.3 Outcome Probabilities with Linear Predictor Functions (Equation (4.20)) 89

A.4 A Proof of the Equivalent Conditions for the D-Optimal Criterion (Chapter 4.2.5) . . . . . . . . . . . . . . . . . . 91

A.5 The Exchange Algorithm for D-Optimality . . . . . . . . . . . 94

A.6 Simulation Study I . . . . . . . . . . . . . . . . . . . . . 97

A.7 Locally Optimal Designs with Various Parameter Values . . . . . . . 101

A.8 A Trivariate Weibull Probability Density . . . . . . . . . . . 105

A.9 Outcome Probabilities (Equation (5.7)) . . . . . . . . 106

A.10 Outcome Probabilities with Regression Terms (Equation (5.8)) . . . . 107

A.11 Outcome Probabilities with Linear Regression Terms (Equation (5.12)) 108

A.12 A Proof of the Equation (5.18) _ . . . . . . . . . . . 109

A.13 The Exchange Algorithm for c-Optimality . . . . . . . . . . . . . 112

A.14 Simulation Study II . . . . . . . . . . . . . . . . . . . . . 114

A.15 A New Multivariate Exponential Probability Density . . . . . . . . 116

A.16 A New Multivariate Weibull Probability Density . . . . . . . . . . . . 117

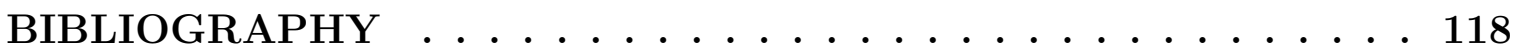

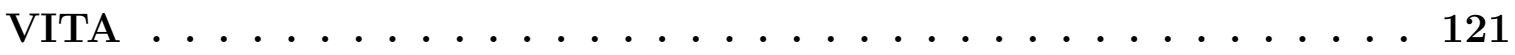




\section{LIST OF TABLES}

Table $\quad$ Page

4.1 Outcome probabilities . . . . . . . . . . . . . . 27

4.2 Efficiency of penalized designs . . . . . . . . . . . . . . . . . 43

4.3 True parameter values for evaluation of robustness . . . . . . . . . . . 50

4.4 Efficiency of locally D-optimal designs under alternative parameter values 51

5.1 Outcomes and their probabilities _. . . . . . . . . . . . 59

6.1 Ternary outcome probabilities . . . . . . . . . . . 79

A.1 True outcome probabilities and simulated outcome probabilities $(n=$

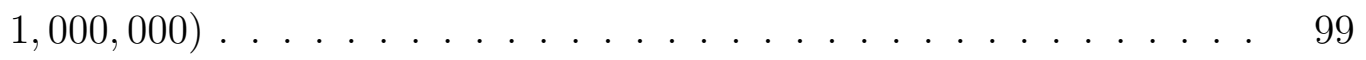




\section{LIST OF FIGURES}

Figure $\quad$ Page

4.1 Bounded penalty functions . . . . . . . . . . . . . . . . . . 32

4.2 IIHS frontal offset crash test . . . . . . . . . . . . . 37

4.3 Marginal Weibull density conditional on stress level for the component 1. $\left(v_{1}^{*}=.8\right) \quad 39$

4.4 Marginal Weibull density conditional on stress level for the component 2. $\left(v_{2}^{*}=.7\right) \quad 39$

4.5 Outcome and marginal probabilities . . . . . . . . . . . . . . . 42

4.6 The locally D-optimal design with a linear predictor . . . . . . . . . . 44

4.7 The penalized D-optimal design $\left(C_{1}=1, C_{2}=1\right)$ with a linear predictor 45

4.8 The penalized D-optimal design $\left(C_{1}=0, C_{2}=1\right)$ with a linear predictor 46

4.9 The penalized D-optimal design $\left(C_{1}=1, C_{2}=0\right)$ with a linear predictor 47

4.10 Probability distributions and D-optimal designs with $\boldsymbol{\theta}_{2}, \boldsymbol{\theta}_{16}$, and $\boldsymbol{\theta}_{10} \quad 54$

4.11 Boxplots of the efficiency of the two-stage adaptive optimal design . . $\quad 55$

5.1 System with decreasing amount of damage . . . . . . . . . . . . 58

5.2 Failure rate changes after component failures at $t_{1}, t_{2}$, and $t_{3}$ given $t_{1}<t_{2}<t_{3}$ under a trivariate exponential model. . . . . . . . . . 63

5.3 c-Optimal design for $c=E S_{\alpha=1} \ldots \ldots \ldots$. . . . . . . . 70

5.4 Boxplots of the efficiency of the two-stage adaptive optimal design . . 73 
6.1 Failure rate change after component failures given $t_{1}<t_{2}<\cdots<t_{R}$ under a multivariate exponential model. . . . . . . . . . . . . . . 76

6.2 A serial system . . . . . . . . . . . . . . . . . . . 78

6.3 The bivariate normal distribution . . . . . . . . . . . . . . 81

6.4 The bivariate Weibull distribution . . . . . . . . . . . . . . . . 81

A.1 Comparisons of bivariate Weibull regression models with the probability histograms of the simulated observations . . . . . . . . . . . . 100

A.2 D-optimal designs varying $\theta_{0} \ldots \ldots \ldots \ldots 1$

A.3 D-optimal designs varying $\theta_{1} \ldots \ldots \ldots$. . . . . . . . . . . 101

A.4 D-optimal designs varying $\theta_{2} \ldots \ldots \ldots$. . . . . . . . . . . . . . . 102

A.5 D-optimal designs varying $\theta_{3} \quad \ldots \ldots$. . . . . . . . . . . . 102

A.6 D-optimal designs varying $\tau$. . . . . . . . . . . . . . 103

A.7 D-optimal designs varying $\beta_{1} \ldots \ldots$. . . . . . . . . . . . 103

A.8 D-optimal designs varying $\beta_{2} \ldots \ldots \ldots$. . . . . . . . . . . . . . . . 104 


\begin{abstract}
In the manufacturing industry, it may be important to study the relationship between machine component failures under stress. Examples include failures such as integrated circuits and memory chips in electronic merchandise given various levels of electronic shock. Such studies are important for the development of new products and for the improvement of existing products. We assume two component systems for simplicity and we assume the joint probability of failures increases with stress as a cumulative bivariate Weibull function. Optimal designs have been developed for two correlated binary responses using the Gumbel model, the bivariate binary Cox model and the bivariate probit model. In all these models, the amount of damage ranges from negative to positive infinity. In the Weibull model, the amount of damage is positive which is natural for experimental factors such as voltage, tension or pressure. First, we describe locally optimal designs under bivariate Weibull assumptions. From among many optimal objective functions, we use the D-optimality criterion which minimizes the inverse of the determinant of information matrix. Since locally optimal designs with non-linear models depend on pre-determined parameter values, misspecified parameter values may lead to designs of the low efficiency. To improve the efficiency of locally optimal designs, we recommend a multi-stage procedure. We show how using a two-stage procedure substantially improves a locally optimal design with misspecified parameters. In addition to D-optimal designs, we describe c-optimal designs under the trivariate Weibull regression model. We assume that the amount of damage decreases sequentially as the stress progresses through the three components. The target stress can be expressed in terms of a linear predictor function, and
\end{abstract}


we evaluate c-optimal designs for optimizing the prediction of the target stress. To compensate for the loss of efficiency of optimal designs with non-linear models, we show a two-stage procedure, and then compare the efficiency of two-stage adaptive optimal designs with single-stage optimal designs. Lastly, we suggest an extension under a multivariate Weibull regression function. 


\section{Chapter 1}

\section{Introduction}

In the manufacturing industry, it is important to study the relationship between machine component failures under stress. Examples include failures such as integrated circuits and memory chips in electronic merchandise given levels of electronic shock, or damage amounts in crash tests given levels of speed. Such studies are important for the development of new products and for the improvement of existing products. To have successful studies, people need efficient experimental designs with a limited budget and time.

Optimal design studies have been applied in various areas to study relationships between responses and covariates. In particular, optimal designs have been developed for two correlated binary responses using well-known distributions including the Gumbel model (Dragalin and Fedorov (2006)), the bivariate binary Cox model (Dragalin, Fedorov, and $\mathrm{Wu}(2006)$ ), and the bivariate probit model (Dragalin, Fedorov, and $\mathrm{Wu}(2008))$. Fedorov and $\mathrm{Wu}(2007)$ compared optimal designs from a bivariate probit model. The bivariate observations were obtained by dichotomizing responses 
from a bivariate normal distribution. They documented the loss in information obtained from dichotomizing random variables as compared with that obtained from the original continuous random variables.

In all these models, random variables range from negative to positive infinity. However, in practice, physical amount of a machine component's failure or damage can't be negative. It must be positive. In the Weibull model, the random variable ranges from zero to positive infinity, which is natural for these experimental studies. This contrasts with the Gumbel model, the bivariate binary Cox model and the bivariate probit model in which binary outcomes are created from outcome measurements that can occur on the whole real line.

Positive responses occur not only in engineering studies, but also in biological and pharmaceutical studies. For example, the outcome measurements discussed in this dissertation are amounts of damage. We can think of other measurements such as the life time of animals or plants, or amount of outcome production.

In this dissertation, we describe optimal designs under multivariate Weibull assumptions. In Chapter 2, background theories and concepts of optimal designs are introduced. In Chapter 3, general methods for constructing bivariate density functions are presented. In Chapter 4, first, this dissertation explains how the bivariate Weibull density was constructed, and then how we created the bivariate Weibull regression model. We assume two dependent component failures for simplicity and assume the probabilities of component failure increase as a cumulative bivariate Weibull regression model with stress. The basic concepts of a locally optimal design and penalized optimal designs for a system with bivariate failures are presented. The general form of the information matrix under a bivariate Weibull regression model is also presented. 
For a practical example, this dissertation introduces a vehicle crash test. We consider two key system components in a crash test: the human component and the machine component for simplicity. Then D-optimal designs are computed using the exchange algorithm (cf. Fedorov and Hackl (1997), Chapter 3) given parameter values. In more practical situations, people may want to find constrained optimal designs that avoid serious damages on machine components at higher stress levels. To address this concern, penalized D-optimal designs are also considered. Since optimal designs with non-linear models depend on the predetermined parameter values, robustness for misspecified parameters is desired. We describe the robustness of locally optimal designs over a wide range of the possible parameter values. We then show that how a locally optimal design with misspecified parameters can be improved substantially using a two-stage procedure. In Chapter 5, we introduce a trivariate exponential density and a trivariate Weibull density. In particular, we assume that the amount of damage to one component is always greater than the amount of damage to the next component. Linear combinations of parameters can be estimated from c-optimal designs with minimum variance. With predictor functions, a covariate can be written as a linear combination of parameters. To estimate the targeted value of a covariate, which here is called an effective stress, with minimum variance efficiently, it is instructive to find a c-optimal experimental design. We describe how to find c-optimal designs under a trivariate Weibull regression function. To find a c-optimal design assuming known parameter values, we also use the exchange algorithm. To improve the efficiency of optimal designs with non-linear models, we show two-stage procedure, and then compare the efficiency of two-stage adaptive optimal designs with single-stage optimal designs. Lastly, this model generalizes to a multivariate exponential density 
and a multivariate Weibull density, and suggests possible extensions. In Chapter 6, we briefly present a future study. 


\section{Chapter 2}

\section{Background Theory}

\subsection{The Design Theory}

Optimal designs have been used to estimate parameters with minimum "variance". Compared to non-optimal designs, one can estimate parameters with fewer experimental runs and smaller costs, but with the same precision. Many optimal objective functions $\Psi$ have been introduced. Denote a design as

$$
\xi=\left\{x_{i}, w_{i}\right\}_{1}^{K},
$$

where

1. $x_{i}$ denotes a support point,

2. $K$ is the total number of support points in $\xi$,

3. $n_{i}$ denotes the "sample size" tested at the support point $x_{i}$, 
4. $n=\sum_{i=1}^{K} n_{i}$,

5. $w_{i}=n_{i} / n$ denotes the proportion of trials at the support point $x_{i}$, and is called a design weight,

6. $x_{i} \in \mathfrak{X}$, a compact sample space.

Designs in which the $n_{i}$ can be any real numbers such that $\sum n_{i}=n$ are called continuous designs. Since ignoring the constraint that $n_{i}$ must be integers simplifies the problem of finding optimal designs, the theory in this paper assumes $\xi$ is a continuous design. Thus we let $w_{i} \in[0,1]$ and $\sum w_{i}=1$. Of course, to implement a continuous design, the $n_{i}$ must be approximated by integers.

In general, the linear model is written as

$$
y=\eta(x, \boldsymbol{\theta})+\epsilon=f^{T}(x) \boldsymbol{\theta}+\epsilon,
$$

where $\boldsymbol{\theta}$ is a $m \times 1$ vector of $m$ unknown parameters and $\eta(x, \boldsymbol{\theta})$ is the mean function of the response $y$. Assuming that $E(\epsilon)=0$, and $\operatorname{Var}(\epsilon)=\sigma^{2}$, it can be re-expressed in a matrix form such as

$$
Y=F \boldsymbol{\theta}+\boldsymbol{\epsilon}
$$

where $Y$ is a $n \times 1$ response, $F$ is a $n \times m$ design matrix, and $\epsilon$ is a $n \times 1$ error matrix. Then the least squares estimator of the parameters is

$$
\widehat{\boldsymbol{\theta}}=\left(F^{T} F\right)^{-1} F^{T} Y
$$


and the covariance matrix of the least squared estimator is

$$
\operatorname{Var} \widehat{\boldsymbol{\theta}}=\sigma^{2}\left(F^{T} F\right)^{-1}
$$

Let $f^{T}\left(x_{j}\right)$ be the $j$ th row of the design matrix $F$. Then the information matrix for $\boldsymbol{\theta}$ can be written as

$$
F^{T} F=\sum_{j=1}^{n} f\left(x_{j}\right) f^{T}\left(x_{j}\right)=n \sum_{i=1}^{K} w_{i} f\left(x_{i}\right) f^{T}\left(x_{i}\right)
$$

Define the average information matrix to be

$$
M(\xi)=\frac{F^{T} F}{n}=\sum_{i=1}^{K} w_{i} f\left(x_{i}\right) f^{T}\left(x_{i}\right) .
$$

The variance of the predicted response is

$$
\operatorname{Var}(\hat{y}(x))=\sigma^{2} f^{T}(x)\left(F^{T} F\right)^{-1} f(x)
$$

and the standardized variance of the predicted response is

$$
d(x, \xi)=\frac{n \operatorname{Var}(\widehat{y}(x))}{\sigma^{2}}=f^{T}(x) M^{-1}(\xi) f(x)
$$

which is later called the sensitivity function for D-optimal designs.

To derive an expression for $\operatorname{Var}(\widehat{\boldsymbol{\theta}})$ in terms of the information in a non-linear model, the mean function $\eta(x, \boldsymbol{\theta})$ is approximated by the Taylor expansion around 
the true parameter $\boldsymbol{\theta}_{t}$ :

$$
\eta(x, \boldsymbol{\theta}) \approx \eta\left(x, \boldsymbol{\theta}_{t}\right)+\left(\boldsymbol{\theta}-\boldsymbol{\theta}_{t}\right) g(x, \boldsymbol{\theta})
$$

where $g(x, \boldsymbol{\theta})=\partial \eta(x, \boldsymbol{\theta}) / \partial \boldsymbol{\theta}$. Then the approximate covariance matrix of the least squared estimator $\widehat{\boldsymbol{\theta}}$ can be written as

$$
\operatorname{Var} \widehat{\boldsymbol{\theta}} \approx \sigma^{2} n^{-1} M^{*-1}(\xi)
$$

where

$$
M^{*}(\xi)=\sum_{i=1}^{K} w_{i} g\left(x_{i}\right) g^{T}\left(x_{i}\right) .
$$

The standardized approximate variance of the predicted response in a non-linear model is

$$
d(x, \xi) \approx g^{T}(x) M^{*-1}(\xi) g(x) .
$$

To achieve the same precision of parameter estimation for given two designs, the following must be satisfied:

$$
\operatorname{Var}_{\xi_{1}} \widehat{\boldsymbol{\theta}}=\operatorname{Var}_{\xi_{2}} \widehat{\boldsymbol{\theta}}
$$

It implies that

$$
\frac{\operatorname{Var}_{\xi_{1}} \widehat{\boldsymbol{\theta}}}{\operatorname{Var}_{\xi_{2}} \widehat{\boldsymbol{\theta}}} \approx \frac{\sigma^{2} n_{1}^{-1} M^{*-1}\left(\xi_{1}\right)}{\sigma^{2} n_{2}^{-1} M^{*-1}\left(\xi_{2}\right)}=1
$$

then

$$
\frac{M^{*}\left(\xi_{2}\right)}{M^{*}\left(\xi_{1}\right)}=\frac{n_{1}}{n_{2}}
$$




\subsection{Optimality Criteria (A Review)}

\subsubsection{D-optimality (Determinant)}

Let $\Psi\{M(\xi)\}$ denote an optimal criterion. The most popular criterion is Doptimality, which seeks to minimize the inverse of the determinant of Fisher's information matrix: $\Psi\{M(\xi)\}=|M(\xi)|^{-1}$, or equivalently maximize the determinant

of Fishers' information matrix of the design. In the asymptotic theory, variance of MLE can be approximated by the inverse of Fisher's information matrix as $n \rightarrow \infty$. Taking the logarithm of the inverse of the determinant of Fisher's information matrix leads to minimization of a convex function, so that any minimum found will be global rather than local. The D-optimal design is defined as:

$$
\begin{aligned}
& \xi^{*}=\arg \min _{\xi \in \Xi(\mathfrak{X})} \Psi\{M(\xi)\}=\arg \min _{\xi \in \Xi(\mathfrak{X})} \log \left|M^{-1}(\xi)\right| \\
= & \arg \min _{\xi \in \Xi(\mathfrak{X})} \log |M(\xi)|^{-1}=\arg \min _{\xi \in \Xi(\mathfrak{X})}\{-\log |M(\xi)|\} .
\end{aligned}
$$

\subsection{2 $D_{A}$-optimality (Generalized D-optimality)}

If we are interested in $p$ linear combinations of unknown parameters such that $\zeta=A^{T} \boldsymbol{\theta}$ where $A$ is a $(m \times p)$ matrix, $p<m$ and $m$ is the number of parameters, then $D_{A}$ optimality criterion is utilized. $D_{A}$ optimality criterion seeks to minimize the determinant of $A^{T} M^{-1}(\xi) A$. Thus the $D_{A}$ optimal design is

$$
\xi^{*}=\arg \min _{\xi \in \Xi(\mathfrak{X})} \log \left|A^{T} M^{-1}(\xi) A\right| .
$$




\subsubsection{A-optimality (Average or Trace)}

$A$-optimality seeks to minimize the trace of the inverse of the information matrix. This criterion results in minimizing the sum or average variance of the estimates of the parameter estimates. Thus the $A$ optimal design is

$$
\xi^{*}=\arg \min _{\xi \in \Xi(\mathfrak{X})} \operatorname{tr} M^{-1}(\xi) .
$$

\subsection{4 c-optimality}

$c$-optimality criterion is used when the interest is in estimating a linear combination of model parameters $c^{T} \boldsymbol{\theta}$ where $c$ is a known vector $m \times 1$ with minimum variance. The $c$-optimal design is

$$
\xi^{*}=\arg \min _{\xi \in \Xi(\mathfrak{X})} c^{T} M^{-1}(\xi) c .
$$

\subsubsection{E-optimality (Eigenvalue)}

E-optimality seeks to minimizes the maximum eigenvalue of the inverse of information matrix. Thus the E-optimal design is

$$
\xi^{*}=\arg \min _{\xi \in \Xi(\mathfrak{X})} \max _{i} \frac{1}{\lambda_{i}},
$$

where $\lambda_{1}, \lambda_{2}, \cdots, \lambda_{m}$ are the eigenvalues of $M(\xi)$. 


\subsubsection{G-optimality}

$G$-optimality minimizes the maximum over the design region $\mathfrak{X}$ of the standardized variance $d(x, \xi)$. The $G$-optimal design is

$$
\xi^{*}=\arg \min _{\xi \in \Xi(\mathfrak{X})} \max _{x \in \mathfrak{X}} d(x, \xi) .
$$

\subsubsection{T-optimality}

$T$-optimality seeks to maximizes the trace of the information matrix. Thus the $T$ optimal design is

$$
\xi^{*}=\arg \max _{\xi \in \Xi(\mathfrak{X})} \operatorname{tr} M(\xi) .
$$

\subsubsection{I-optimality (Integrated)}

$I$-optimality seeks to minimize the average of $d(x, \xi)$ over $\mathfrak{X}$. Thus the $I$ optimal design is

$$
\xi^{*}=\arg \min _{\xi \in \Xi(\mathfrak{X})} \frac{\int_{\mathfrak{X}} d(x, \xi) d x}{\int_{\mathfrak{X}} d x} .
$$

\subsubsection{V-optimality (Variance)}

$V$-optimality seeks to minimize the average of $d(x, \xi)$ over a set of $l$ specific design points. Thus the $G, I$, and $V$ optimality criteria find designs to minimize one aspect of $d(x, \xi)$. 


\subsubsection{The General Equivalence Theorem}

The basic idea behind the General Equivalence Theorem is that derivatives are zero at the minimum of a smooth function over a unconstrained region. The function to be minimized depends on the design $\xi$ through the information matrix $M(\xi)$. Let

the design $\bar{\xi}$ put unit mass at the point $x: \bar{\xi}=\{x, 1\}$, and let the design $\xi^{\prime}$ be given by

$$
\xi^{\prime}=(1-\alpha) \xi+\alpha \bar{\xi}
$$

Then

$$
M\left(\xi^{\prime}\right)=(1-\alpha) M(\xi)+\alpha M(\bar{\xi}) .
$$

The derivative of $\Psi$ in the direction $\bar{\xi}$ is

$$
\phi(x, \xi)=\lim _{\alpha \rightarrow 0^{+}} \frac{[\Psi\{(1-\alpha) M(\xi)+\alpha M(\bar{\xi})\}-\Psi\{M(\xi)\}]}{\alpha} .
$$

Then the General Equivalence Theorem states three equivalent conditions.

1. $\xi^{*}=\arg \min _{\xi \in \Xi(\mathfrak{X})} \Psi\{M(\xi)\}$.

2. $\xi^{*}=\arg \max _{\xi \in \Xi(\mathfrak{X})} \min _{x \in \mathfrak{X}} \phi(x, \xi)$.

3. $\min _{x \in \mathfrak{X}} \phi\left(x, \xi^{*}\right)=0$ at the support points of the optimal design.

\subsubsection{Carathéodory's Theorem}

Carathéodory's Theorem states that if a point $x$ of $R^{d}$ lies in the convex hull of a set $P$, there is a subset $P^{\prime}$ of $P$ consisting of $d+1$ or fewer points such that $x$ lies in the convex hull of $P^{\prime}$. In other words, a point $x$ lies in the convex hull of a set $P$ may 
be represented as a convex linear combination of not more than $d+1$ points in $P$. If $x$ is a boundary point, then it may be represented as a convex linear combination of not more than $d$ points in $P$.

Let $\mathfrak{M}(\mathfrak{X})$ be a convex hull of the set $\left\{M(x)=f(x) f^{T}(x) \in R^{\frac{m(m+1)}{2}}: x \in \mathfrak{X}\right\}$. By Carathéodory's Theorem, any average information matrix may be represented as a convex linear combination of not more than $K_{0}$ elements $M(x)$ :

$$
M(\xi)=\sum_{i=1}^{K_{0}} w_{i} M\left(x_{i}\right), \sum_{i=1}^{K_{0}} w_{i}=1
$$

where $K_{0} \leq \frac{m(m+1)}{2}+1$, and $K_{0} \leq \frac{m(m+1)}{2}$ for boundary points.

It states that an optimal design $\xi$ with an average information matrix $M(\xi)$ contains not more than $\frac{m(m+1)}{2}+1$ design points with non-zero weight $w_{i}$. If $M(\xi)$ is a boundary point of $\mathfrak{M}(\mathfrak{X})$ then the optimal design $\xi$ contains not more than $\frac{m(m+1)}{2}$ design points. 


\section{Chapter 3}

\section{Methods for Constructing Bivariate Density Functions}

\subsection{The Basic Idea}

\subsubsection{Frechet Bounds}

In considering the general problem of constructing bivariate distributions $H$, the condition is given as follow. Let $f$ and $g$ be marginal probability density functions. For given marginal distribution functions $F$ and $G$, in order to have its probability density function be non-negative everywhere, limits of a joint distribution function $H$ must satisfy the following requirement (Hoeffding (1940) and Frechet (1951)):

$$
H^{-}(x, y) \leq H(x, y) \leq H^{+}(x, y)
$$


where

$$
H^{+}(x, y)=\min [F(x), G(y)]
$$

and

$$
H^{-}(x, y)=\max [F(x)+G(y)-1,0] .
$$

\subsubsection{Transformations}

Let $h(x, y)$ be a bivariate density and we have two new variables $A(X, Y)$ and $B(X, Y)$. The joint density of $A$ and $B$ is then

$$
h[x(a, b), y(x, y)]|J|
$$

where $J$ is the Jacobian, given by

$$
J=\left|\begin{array}{ll}
\frac{\partial x}{\partial a} & \frac{\partial x}{\partial b} \\
\frac{\partial y}{\partial a} & \frac{\partial y}{\partial b}
\end{array}\right|
$$

\subsection{Product of a Conditional Density and a Marginal Density}

A bivariate probability density function can be constructed as the product of a marginal probability density function and a conditional probability density function such as

$$
h(x, y)=f(x) g(y \mid x) .
$$




\subsection{The Monotone Transformation Method}

Let $H(x, y)$ be a bivariate distribution and apply monotone transformations $X \rightarrow$ $X^{*}$ and $Y \rightarrow Y^{*}$. Then we have a new bivariate distribution $H^{*}\left(x^{*}, y^{*}\right)$.

Example 1) Suppose $(\mathrm{X}, \mathrm{Y})$ has a bivariate normal distribution, then $\left(e^{x}, e^{y}\right)$ has a bivariate lognormal distribution and $\left(\left(e^{x} /\left(1+e^{x}+e^{y}\right)\right),\left(e^{y} /\left(1+e^{x}+e^{y}\right)\right)\right)$ has a bivariate logistic-normal distribution.

Example 2) Freund (1961) constructed the bivariate exponential density:

$$
f\left(t_{1}, t_{2}\right)= \begin{cases}\beta_{1}\left(\beta_{5}+\beta_{3}\right) e^{-\left(\beta_{5}+\beta_{3}\right) t_{2}-\left(\beta_{1}+\beta_{2}-\beta_{5}\right) t_{1}} & \text { for } 0<t_{1}<t_{2}<\infty \\ \beta_{2}\left(\beta_{4}+\beta_{3}\right) e^{-\left(\beta_{4}+\beta_{3}\right) t_{1}-\left(\beta_{1}+\beta_{2}-\beta_{4}\right) t_{2}} & \text { for } 0<t_{2}<t_{1}<\infty \\ \beta_{3} e^{-\left(\beta_{1}+\beta_{2}+\beta_{3}\right) t_{1}} & \text { for } 0<t_{1}=t_{2}<\infty .\end{cases}
$$

By the transformations $T_{1}=Y^{\tau}$ and $T_{2}=Z^{\tau}$, we can get the bivariate Weibull density as follows (Hanagal (2005)):

$$
f(y, z)= \begin{cases}\beta_{1}\left(\beta_{5}+\beta_{3}\right) \tau^{2}(y z)^{\tau-1} e^{-\left(\beta_{5}+\beta_{3}\right) z^{\tau}-\left(\beta_{1}+\beta_{2}-\beta_{5}\right) y^{\tau}} & \text { for } 0<y<z<\infty \\ \beta_{2}\left(\beta_{4}+\beta_{3}\right) \tau^{2}(y z)^{\tau-1} e^{-\left(\beta_{4}+\beta_{3}\right) y^{\tau}-\left(\beta_{1}+\beta_{2}-\beta_{4}\right) z^{\tau}} & \text { for } 0<z<y<\infty \\ \beta_{3} \tau y^{\tau-1} e^{-\left(\beta_{1}+\beta_{2}+\beta_{3}\right) y^{\tau}} & \text { for } 0<y=z<\infty .\end{cases}
$$

Taking logarithm of the bivariate exponential variables, i.e., $X_{1}=\log T_{1}$ and $X_{2}=\log T_{2}$, we can get a bivariate extreme value density:

$$
f\left(x_{1}, x_{2}\right)= \begin{cases}e^{x_{1}+\log \beta_{1}+x_{2}+\log \left(\beta_{5}+\beta_{3}\right)-e^{x_{1}+\log \left(\beta_{1}+\beta_{2}-\beta_{5}\right)}-e^{x_{2}+\log \left(\beta_{5}+\beta_{3}\right)}} & \text { for } 0<x_{1}<x_{2}<\infty \\ e^{x_{2}+\log \beta_{2}+x_{1}+\log \left(\beta_{4}+\beta_{3}\right)-e^{x_{2}+\log \left(\beta_{1}+\beta_{2}-\beta_{4}\right)}-e^{x_{1}+\log \left(\beta_{4}+\beta_{3}\right)}} & \text { for } 0<x_{2}<x_{1}<\infty \\ e^{x_{1}+\log \beta_{3}-e^{x_{1}}+\log \left(\beta_{1}+\beta_{2}+\beta_{3}\right)} & \text { for } 0<x_{1}=x_{2}<\infty .\end{cases}
$$


Example 3) If the joint survival function with exponential marginals is given by

$$
\bar{F}(x, y)=P(X>x, Y>y)=\exp \left[-\lambda_{1} x-\lambda_{2} y-\lambda_{12} \max (x, y)\right]
$$

where $\lambda_{1}, \lambda_{2}, \lambda_{12}>0$ (Marshall, Olkin (1967)), then the distribution of $\left(X^{\frac{1}{\beta}}, Y^{\frac{1}{\gamma}}\right)$ is a joint survival function with Weibull marginals:

$$
\bar{F}=\exp \left[-\lambda_{1} x^{\beta}-\lambda_{2} y^{\gamma}-\lambda_{12} \max \left(x^{\beta}, y^{\gamma}\right)\right] .
$$

\subsection{Methods for Constructing Copulas}

A copula is a general way of formulating a bivariate distribution in such a way that various general types of dependence can be represented. Therefore, copulas play an very important role in constructing bivariate distributions. If the copula $\mathrm{C}$ is determined, then a bivariate distribution $H$ with given marginal distributions $F$ and $G$ can be generated by Sklar's theorem (1959) that $H(x, y)=C(F(x), G(y))$.

\subsubsection{The Univariate Function Method}

Durante (2007) introduced a family of symmetric copulas from a univariate function such as $f:[0,1] \rightarrow[0,1]$. The function $f$ is continuous and differentiable except at finitely many points. Let

$$
C(x, y)=\min (x, y) f(\max (x, y)) .
$$

Then $C$ is a copula if and only if 
1. $f(1)=1$,

2. $f$ is an increasing function,

3. The function $f(t) / t$ is decreasing on $(0,1]$.

\subsubsection{The Inversion Method (Marginal Transformation Method)}

Copulas can be constructed through inverse probability integral transforms of the marginals $F^{-1}(u)=x$ and $G^{-1}(v)=y$. For a given bivariate distribution $H$ with continuous marginal distributions $F$ and $G$, a copula can be obtained:

$$
C(u, v)=H\left(F^{-1}(u), G^{-1}(v)\right) .
$$

\subsubsection{Geometric Methods}

Without reference to distribution functions or random variables, we can obtain a copula via the C-Volume of rectangles in $[0,1] \times[0,1]$.

\subsubsection{Algebraic Methods}

Two well-known families of copulas, the Plackett $(2 \times 2$ contingency tables $)$ and Ali-Mikhail-Haq (survival odds ratio) families, were constructed using an algebraic relationship between the joint distribution and its univariate marginal distributions. 


\subsubsection{The Rüschendorf's Method}

Rüschendorf (1985) introduced a new method of constructing a copula as follows:

Step1. Start with a function $f^{1}(u, v)$ such that

$$
\int_{0}^{1} \int_{0}^{1} f^{1}(u, v) d u d v=0, \quad \int_{0}^{1} f^{1}(u, v) d u=0, \text { and } \int_{0}^{1} f^{1}(u, v) d v=0 .
$$

Step2. Then a density of a copula is $c(u, v)=1+f^{1}(u, v)$. But there is the constraint that $c(u, v)$ must be non-negative. If $c(u, v)$ is negative, but $f^{1}$ is bounded, then we need a constant $\alpha$ such that $1+\alpha f^{1}$ is positive.

One can find a density of a copula from an arbitrary real integrable function $f$ on the unit square:

$$
V=\int_{0}^{1} \int_{0}^{1} f(u, v) d u d v, f_{1}(u)=\int_{0}^{1} f(u, v) d v, f_{2}(v)=\int_{0}^{1} f(u, v) d u
$$

then, $f^{1}=f-f_{1}-f_{2}+V$.

\subsubsection{Constructing a Copula from a Distortion Function}

One can use a distortion function $\phi$ that maps $[0,1]$ onto $[0,1]$, with $\phi(0)=0$, $\phi(1)=1$, and $\phi$ is an increasing function (Frees and Valdez (1998)). From a bivariate distribution:

$$
H(x, y)=C(F(x), G(y))
$$


we define another distribution function $H^{*}(x, y)$ via a function $\phi$ :

$$
H^{*}(x, y)=\phi[H(x, y)]
$$

with marginals $F^{*}(x)=\phi(F(x))$ and $G^{*}(y)=\phi(G(y))$. The new copula is then

$$
C^{*}(u, v)=\phi\left[C\left(\phi^{-1}(u), \phi^{-1}(v)\right)\right] .
$$

\subsection{Uniform Representation}

The innovative approach to formulating a bivariate distribution using a copula is based on the idea that a simple transformation can be made of each marginal variable in such a way that each transformed marginal variable has a uniform distribution over the range 0 to 1 . For example, let $H$ be a bivariate distribution,

$$
H=x y[1+\alpha(1-x)(1-y)]
$$

(Farlie-Gumbel-Morgenstern copula (Morgenstern (1956)))

for $0<x<1,0<y<1$, and $0<\alpha<1$. If $y=1$, then the distribution of $X$ is uniform, and if $x=1$, then the distribution of $Y$ is uniform. We can replace $x$ by $1-e^{-x}$ and $y$ by $1-e^{-y}$ (marginals to be exponential) to obtain a bivariate exponential distribution (Gumbel (1960)). 


\subsection{Marshall and Olkin's Mixture Method}

Marshall and Olkin (1988) used mixtures to generate bivariate distributions:

$$
H(u, v)=\iint K\left(F^{\theta_{1}}, G^{\theta_{2}}\right) d \Lambda\left(\theta_{1}, \theta_{2}\right)
$$

where $K$ is a copula, $\Lambda$ is a mixing distribution. We can generate various distributions with marginals $F$ and $G$ as parameters. Note that $F$ and $G$ are not necessarily the marginals of $H$. 


\section{Chapter 4}

\section{A New Bivariate Weibull Function}

\subsection{A New Bivariate Weibull Regression Model}

\subsubsection{A Bivariate Weibull Density}

Freund (1961) used conditional densities with marginal densities to construct a bivariate exponential density. Let $T_{1}$ and $T_{2}$ have exponential probability densities:

$$
\begin{aligned}
& f\left(t_{1}\right)=\beta_{1} e^{-\beta_{1} t_{1}} \text { for } t_{1}>0 ; \\
& f\left(t_{2}\right)=\beta_{2} e^{-\beta_{2} t_{2}} \text { for } t_{2}>0 .
\end{aligned}
$$

Then the probability that $T_{1}$ fails (occurs) at $t_{1}$ and $T_{2}$ has not yet failed is

$$
\left.\frac{\partial}{\partial t} P\left(T_{1}<t, T_{2}>t_{1}\right)\right|_{t=t_{1}}=\beta_{1} e^{-\beta_{1} t_{1}} \times e^{-\beta_{2} t_{1}}=\beta_{1} e^{-\left(\beta_{1}+\beta_{2}\right) t_{1}} .
$$


Specify, furthermore, that the probability density of $T_{2}$ given that $T_{1}$ fails first at $t_{1}$ is

$$
\left.\frac{\partial}{\partial t} P\left(T_{2}<t \mid T_{2}>t_{1}\right)\right|_{t=t_{2}>t_{1}}=\beta_{5} e^{-\beta_{5}\left(t_{2}-t_{1}\right)} \quad \text { for } 0<t_{1}<t_{2} .
$$

Multiplying (4.1) by (4.2), the bivariate exponential density for $0<t_{1}<t_{2}$ is

$$
f\left(T_{1}=t_{1}, T_{2}=t_{2}\right)=\beta_{1} e^{-\left(\beta_{1}+\beta_{2}\right) t_{1}} \times \beta_{5} e^{-\beta_{5}\left(t_{2}-t_{1}\right)} \text { for } 0<t_{1}<t_{2}<\infty .
$$

Analogously, the probability that $T_{2}$ fails at $t_{2}$ and $T_{1}$ has not yet failed is

$$
\left.\frac{\partial}{\partial t} P\left(T_{2}<t, T_{1}>t_{2}\right)\right|_{t=t_{2}}=\beta_{2} e^{-\beta_{2} t_{2}} \times e^{-\beta_{1} t_{2}}=\beta_{2} e^{-\left(\beta_{1}+\beta_{2}\right) t_{2}} .
$$

The probability density of $T_{1}$ given that $T_{2}$ fails first at $t_{2}$ is

$$
\left.\frac{\partial}{\partial t} P\left(T_{1}<t \mid T_{1}>t_{2}\right)\right|_{t=t_{1}>t_{2}}=\beta_{4} e^{-\beta_{4}\left(t_{1}-t_{2}\right)} \quad \text { for } 0<t_{2}<t_{1}
$$

Multiplying (4.3) by (4.4), the bivariate exponential density for $0<t_{2}<t_{1}$ is

$$
f\left(T_{1}=t_{1}, T_{2}=t_{2}\right)=\beta_{2} e^{-\left(\beta_{1}+\beta_{2}\right) t_{2}} \times \beta_{4} e^{-\beta_{4}\left(t_{1}-t_{2}\right)} \quad \text { for } 0<t_{2}<t_{1}<\infty
$$

In summary, the bivariate exponential distribution is

$$
f\left(t_{1}, t_{2}\right)= \begin{cases}\beta_{1} e^{-\left(\beta_{1}+\beta_{2}\right) t_{1}} \times \beta_{5} e^{-\beta_{5}\left(t_{2}-t_{1}\right)} & \text { for } 0<t_{1}<t_{2}<\infty \\ \beta_{2} e^{-\left(\beta_{1}+\beta_{2}\right) t_{2}} \times \beta_{4} e^{-\beta_{4}\left(t_{1}-t_{2}\right)} & \text { for } 0<t_{2}<t_{1}<\infty\end{cases}
$$




$$
= \begin{cases}\beta_{1}\left(\beta_{5}\right) e^{-\left(\beta_{5}\right) t_{2}-\left(\beta_{1}+\beta_{2}-\beta_{5}\right) t_{1}} & \text { for } 0<t_{1}<t_{2}<\infty \\ \beta_{2}\left(\beta_{4}\right) e^{-\left(\beta_{4}\right) t_{1}-\left(\beta_{1}+\beta_{2}-\beta_{4}\right) t_{2}} & \text { for } 0<t_{2}<t_{1}<\infty .\end{cases}
$$

Marshall-Olkin (1967) introduced dependency between exponential random variables using a copula method:

$$
\begin{aligned}
\bar{F}_{M}\left(t_{1}, t_{2}\right) & =P\left[T_{1}>t_{1}, T_{2}>t_{2}\right] \\
& =\exp \left[-\beta_{1} t_{1}-\beta_{2} t_{2}-\beta_{3} \max \left(t_{1}, t_{2}\right)\right], \quad \beta_{1}, \beta_{2}, \beta_{3}>0 .
\end{aligned}
$$

Proschan-Sullo (1974) repeated steps taken by Freund, starting with marginal distribution $T_{1}$ and $T_{2}$ obtained from Marshall-Olkin to get a bivariate exponential density with three parameters.

$$
f\left(t_{1}, t_{2}\right)= \begin{cases}\beta_{1}\left(\beta_{5}+\beta_{3}\right) e^{-\left(\beta_{5}+\beta_{3}\right) t_{2}-\left(\beta_{1}+\beta_{2}-\beta_{5}\right) t_{1}} & \text { for } 0<t_{1}<t_{2}<\infty \\ \beta_{2}\left(\beta_{4}+\beta_{3}\right) e^{-\left(\beta_{4}+\beta_{3}\right) t_{1}-\left(\beta_{1}+\beta_{2}-\beta_{4}\right) t_{2}} & \text { for } 0<t_{2}<t_{1}<\infty \\ \beta_{3} e^{-\left(\beta_{1}+\beta_{2}+\beta_{3}\right) t_{1}} & \text { for } 0<t_{1}=t_{2}<\infty\end{cases}
$$

The marginal density functions of (4.6) can be written as weighted univariate exponential density functions:

$$
\begin{aligned}
& f\left(t_{1}\right)=a_{1}\left(\beta_{4}+\beta_{3}\right) e^{-\left(\beta_{4}+\beta_{3}\right) t_{1}}+\left(1-a_{1}\right)\left(\beta_{1}+\beta_{2}+\beta_{3}\right) e^{-\left(\beta_{1}+\beta_{2}+\beta_{3}\right) t_{1}} \\
& f\left(t_{2}\right)=a_{2}\left(\beta_{5}+\beta_{3}\right) e^{-\left(\beta_{5}+\beta_{3}\right) t_{2}}+\left(1-a_{2}\right)\left(\beta_{1}+\beta_{2}+\beta_{3}\right) e^{-\left(\beta_{1}+\beta_{2}+\beta_{3}\right) t_{2}}
\end{aligned}
$$

where $a_{1}=\left(\frac{\beta_{2}}{\beta_{1}+\beta_{2}-\beta_{4}}\right)$ and $a_{2}=\left(\frac{\beta_{1}}{\beta_{1}+\beta_{2}-\beta_{5}}\right)$. If $\beta_{1}=\beta_{4}, \beta_{2}=\beta_{5}$, and $\beta_{3}=0$, then $a_{1}=a_{2}=1$ and (4.6) reduces to two independent univariate exponential density functions. 
David D. Hanagal (2005) proposed a bivariate Weibull density by means of the transformations $T_{1}=Y^{\tau}$ and $T_{2}=Z^{\tau}$ from the bivariate exponential density proposed by Proschan and Sullo (1974).

$$
f(y, z)= \begin{cases}\beta_{1}\left(\beta_{5}+\beta_{3}\right) \tau^{2}(y z)^{\tau-1} e^{-\left(\beta_{5}+\beta_{3}\right) z^{\tau}-\left(\beta_{1}+\beta_{2}-\beta_{5}\right) y^{\tau}} & \text { for } 0<y<z<\infty \\ \beta_{2}\left(\beta_{4}+\beta_{3}\right) \tau^{2}(y z)^{\tau-1} e^{-\left(\beta_{4}+\beta_{3}\right) y^{\tau}-\left(\beta_{1}+\beta_{2}-\beta_{4}\right) z^{\tau}} & \text { for } 0<z<y<\infty \\ \beta_{3} \tau y^{\tau-1} e^{-\left(\beta_{1}+\beta_{2}+\beta_{3}\right) y^{\tau}} & \text { for } 0<y=z<\infty\end{cases}
$$

The marginal density functions of the bivariate Weibull density (4.8) are weighted univariate Weibull densities:

$$
\begin{aligned}
& f(y)=a_{1}\left(\beta_{4}+\beta_{3}\right) \tau y^{\tau-1} e^{-\left(\beta_{4}+\beta_{3}\right) y^{\tau}}+\left(1-a_{1}\right)\left(\beta_{1}+\beta_{2}+\beta_{3}\right) \tau y^{\tau-1} e^{-\left(\beta_{1}+\beta_{2}+\beta_{3}\right) y^{\tau}} \\
& f(z)=a_{2}\left(\beta_{5}+\beta_{3}\right) \tau z^{\tau-1} e^{-\left(\beta_{5}+\beta_{3}\right) z^{\tau}}+\left(1-a_{2}\right)\left(\beta_{1}+\beta_{2}+\beta_{3}\right) \tau z^{\tau-1} e^{-\left(\beta_{1}+\beta_{2}+\beta_{3}\right) z^{\tau}}
\end{aligned}
$$

where $a_{1}=\left(\frac{\beta_{2}}{\beta_{1}+\beta_{2}-\beta_{4}}\right)$ and $a_{2}=\left(\frac{\beta_{1}}{\beta_{1}+\beta_{2}-\beta_{5}}\right)$. These marginals reduce to (4.7) when $\tau=1$.

\subsubsection{A New Bivariate Weibull Regression Model}

Let $T$ have a univariate exponential density function $f(t)=\beta e^{-\beta t}$ for $0<t<\infty$. The univariate Weibull density function, $f(y)=\beta \tau y^{\tau-1} e^{-\beta y^{\tau}}$, for $0<y<\infty$, can be obtained from the transformation $Y=T^{1 / \tau} . W=\log \left(\beta Y^{\tau}\right)$ follows the standard extreme value distribution $f(w)=e^{w-e^{w}}$. A common regression model is obtained by introducing covariates as $-\log \beta=\boldsymbol{\vartheta}^{\boldsymbol{T}} \boldsymbol{f}(\boldsymbol{x})$ which implies $\tau \log Y=\boldsymbol{\vartheta}^{\boldsymbol{T}} \boldsymbol{f}(\boldsymbol{x})+W$ (cf. Klein and Moeschberger p.39, 2003). This regression model motivates our new 
bivariate Weibull regression model.

Similar to the univariate Weibull, the scale parameters in the bivariate Weibull distribution (4.8) can be written as a function of regression parameters:

$$
\begin{aligned}
& -\log \left(\beta_{5}+\beta_{3}\right)=\eta_{1}\left(\boldsymbol{x} ; \boldsymbol{\vartheta}_{1}\right) \\
& -\log \left(\beta_{4}+\beta_{3}\right)=\eta_{2}\left(\boldsymbol{x} ; \boldsymbol{\vartheta}_{2}\right) \\
& -\log \left(\beta_{1}+\beta_{2}+\beta_{3}\right)=\eta_{3}\left(\boldsymbol{x} ; \boldsymbol{\vartheta}_{3}\right),
\end{aligned}
$$

where $\eta_{1}=\eta_{1}\left(\boldsymbol{x} ; \boldsymbol{\vartheta}_{1}\right), \eta_{2}=\eta_{2}\left(\boldsymbol{x} ; \boldsymbol{\vartheta}_{2}\right)$, and $\eta_{3}=\eta_{3}\left(\boldsymbol{x} ; \boldsymbol{\vartheta}_{3}\right)$ are functions which, in general, may be nonlinear; $\boldsymbol{\vartheta}_{1}, \boldsymbol{\vartheta}_{2}$, and $\boldsymbol{\vartheta}_{3}$ may be vectors of parameters, and $\boldsymbol{x}$ may be a vector of covariates.

Because scale parameters must be positive, it follows from (4.8) that the $\boldsymbol{\vartheta}_{1}, \boldsymbol{\vartheta}_{2}$, and $\boldsymbol{\vartheta}_{3}$ must satisfy the conditions such that $\left(\beta_{1}+\beta_{2}-\beta_{5}\right)=e^{-\eta_{3}\left(\boldsymbol{x} ; \boldsymbol{\vartheta}_{3}\right)}-e^{-\eta_{1}\left(\boldsymbol{x} ; \boldsymbol{\vartheta}_{1}\right)}>$ 0 , and $\left(\beta_{1}+\beta_{2}-\beta_{4}\right)=e^{-\eta_{3}\left(\boldsymbol{x} ; \boldsymbol{\vartheta}_{3}\right)}-e^{-\eta_{2}\left(\boldsymbol{x} ; \boldsymbol{\vartheta}_{2}\right)}>0$ in the sample space.

\subsection{Optimal Experimental Designs with Bivariate Outcomes}

\subsubsection{Two Dependent Binary Outcomes}

This section considers two dependent binary outcomes. Let $U$ and $V$ denote the outcomes for the first and second system components, respectively, with 0 indicating no failure and 1 indicating failure. Let $p_{u v}(x, \boldsymbol{\theta})=\operatorname{Pr}(U=u, V=v \mid X=x),\{u, v\} \in$ $\{0,1\}$ denote the outcome probabilities given a stress $x$, where $\boldsymbol{\theta}$ denotes a vector of all the model parameters. Dropping the reference to $x$ and $\boldsymbol{\theta}$ when not confusing, 
these probabilities $p_{u v}=p_{u v}(x, \boldsymbol{\theta})$ can be arranged in the common contingency table format together with their associated marginal probabilities. See Table 4.1.

\begin{tabular}{|c|cl|c|}
\hline 1st Component & 0 (Success) & 1 (Failure) & \\
\hline 2nd Component & $p_{00}$ & $p_{10}$ & $p_{\cdot 0}$ \\
1 (Fuccess) & $p_{01}$ & $p_{11}$ & $p_{\cdot 1}$ \\
\hline & $p_{0}$. & $p_{1}$ & 1 \\
\hline
\end{tabular}

Table 4.1: Outcome probabilities

Let $Y$ and $Z$ denote amount of damage on component 1 and component 2, respectively, and let $f(y, z \mid x, \boldsymbol{\theta})$ be the bivariate Weibull regression model whose vector of model parameters $\boldsymbol{\theta}$ is described in Section 4.1. Now suppose failures (fatal damages) are defined by dichotomizing damage measurements $Y$ and $Z$ :

$$
\begin{aligned}
& U=\left\{\begin{array}{l}
0 \text { (No failure for the component } 1), \text { if } Y<v_{1}^{*} \\
1 \text { (Failure for the component } 1), \text { otherwise, }
\end{array}\right. \\
& V=\left\{\begin{array}{l}
0 \text { (No failure for the component } 2), \text { if } Z<v_{2}^{*} \\
1 \text { (Failure for the component } 2), \text { otherwise, }
\end{array}\right.
\end{aligned}
$$

where $v_{1}^{*}$ and $v_{2}^{*}$ are predetermined cutoff values. Then the probabilities in Table 4.1 are

$$
\begin{aligned}
& p_{00}=\int_{0}^{v_{1}^{*}} \int_{0}^{v_{2}^{*}} f(y, z \mid x, \boldsymbol{\theta}) d y d z ; \\
& p_{01}=\int_{0}^{v_{1}^{*}} \int_{v_{2}^{*}}^{\infty} f(y, z \mid x, \boldsymbol{\theta}) d z d y \\
& p_{10}=\int_{v_{1}^{*}}^{\infty} \int_{0}^{v_{2}^{*}} f(y, z \mid x, \boldsymbol{\theta}) d z d y \\
& p_{11}=\int_{v_{1}^{*}}^{\infty} \int_{v_{2}^{*}}^{\infty} f(y, z \mid x, \boldsymbol{\theta}) d y d z .
\end{aligned}
$$

The outcome probabilities in Table 4.1 under the bivariate Weibull model can be 
computed from (4.8). For example, when $v_{1}^{*}>v_{2}^{*}$,

$$
\begin{aligned}
p_{00} & =\int_{0}^{v_{2}^{*}} \int_{0}^{z} \beta_{1}\left(\beta_{5}+\beta_{3}\right) \tau^{2}(y z)^{\tau-1} e^{-\left(\beta_{5}+\beta_{3}\right) z^{\tau}-\left(\beta_{1}+\beta_{2}-\beta_{5}\right) y^{\tau}} d y d z \\
& +\int_{0}^{v_{2}^{*}} \int_{z}^{v_{1}^{*}} \beta_{2}\left(\beta_{4}+\beta_{3}\right) \tau^{2}(y z)^{\tau-1} e^{-\left(\beta_{4}+\beta_{3}\right) y^{\tau}-\left(\beta_{1}+\beta_{2}-\beta_{4}\right) z^{\tau}} d y d z \\
& +\int_{0}^{v_{2}^{*}} \beta_{3} \tau y^{\tau-1} e^{-\left(\beta_{1}+\beta_{2}+\beta_{3}\right) y^{\tau}} d y .
\end{aligned}
$$

The other probabilities can be expressed similarly. All our calculations assume $v_{1}^{*}>$ $v_{2}^{*}$. We could do the converse. But if you label the components so that $v_{1}^{*}>v_{2}^{*}$ is true, one doesn't need the calculations for $v_{1}^{*}<v_{2}^{*}$.

The total law of probability applied to (4.8) requires the following:

$$
\begin{array}{ll}
\int_{0}^{\infty} \int_{0}^{z} \beta_{1}\left(\beta_{5}+\beta_{3}\right) \tau^{2}(y z)^{\tau-1} e^{-\left(\beta_{5}+\beta_{3}\right) z^{\tau}-\left(\beta_{1}+\beta_{2}-\beta_{5}\right) y^{\tau}} d y d z=\frac{\beta_{1}}{\beta_{1}+\beta_{2}+\beta_{3}} & \text { for } 0<y<z<\infty \\
\int_{0}^{\infty} \int_{0}^{y} \beta_{2}\left(\beta_{4}+\beta_{3}\right) \tau^{2}(y z)^{\tau-1} e^{-\left(\beta_{4}+\beta_{3}\right) y^{\tau}-\left(\beta_{1}+\beta_{2}-\beta_{4}\right) z^{\tau}} d z d y=\frac{\beta_{2}}{\beta_{1}+\beta_{2}+\beta_{3}} & \text { for } 0<z<y<\infty ; \\
\int_{0}^{\infty} \beta_{3} \tau y^{\tau-1} e^{-\left(\beta_{1}+\beta_{2}+\beta_{3}\right) y^{\tau}} d y=\frac{\beta_{3}}{\beta_{1}+\beta_{2}+\beta_{3}} & \text { for } 0<y=z<\infty
\end{array}
$$

These conditions imply that $\beta_{1}, \beta_{2}$, and $\beta_{3}$ must be positive; $\beta_{4}$ and $\beta_{5}$ can be negative, but $\beta_{4}$ and $\beta_{5}$ must be smaller than $\beta_{3}$ because scale parameters of Weibull density (4.9) must be positive.

Let $\boldsymbol{\theta}=\left\{\boldsymbol{\vartheta}_{1}, \boldsymbol{\vartheta}_{2}, \boldsymbol{\vartheta}_{3}, \tau, \beta_{1}, \beta_{2}\right\}$. Inserting the regression coefficients (4.10) into 
(4.12) yields

$$
\begin{aligned}
p_{00}(x \mid \boldsymbol{\theta})= & \frac{\beta_{1}}{e^{-\eta_{3}\left(\boldsymbol{x} ; \boldsymbol{\vartheta}_{3}\right)}-e^{-\eta_{1}\left(\boldsymbol{x} ; \boldsymbol{\vartheta}_{1}\right)}}\left(1-\exp \left(-\left(e^{-\eta_{1}\left(\boldsymbol{x} ; \boldsymbol{\vartheta}_{1}\right)}\right) v_{2}^{* \tau}\right)\right) \\
& -\frac{\beta_{2}}{e^{-\eta_{3}\left(\boldsymbol{x} ; \boldsymbol{\vartheta}_{3}\right)}-e^{-\eta_{2}\left(\boldsymbol{x} ; \boldsymbol{\vartheta}_{2}\right)}}\left(1-\exp \left(-\left(e^{-\eta_{3}\left(\boldsymbol{x} ; \boldsymbol{\vartheta}_{3}\right)}-e^{-\eta_{2}\left(\boldsymbol{x} ; \boldsymbol{\vartheta}_{2}\right)}\right) v_{2}^{* \tau}\right)\right) \\
& \times \exp \left(-\left(e^{-\eta_{2}\left(\boldsymbol{x} ; \boldsymbol{\vartheta}_{2}\right)}\right) v_{1}^{* \tau}\right) \\
& +\frac{e^{-\eta_{3}\left(\boldsymbol{x} ; \boldsymbol{\vartheta}_{3}\right)}-e^{-\eta_{1}\left(\boldsymbol{x} ; \boldsymbol{\vartheta}_{1}\right)}-\beta_{1}}{e^{-\eta_{3}\left(\boldsymbol{x} ; \boldsymbol{\vartheta}_{3}\right)}-e^{-\eta_{1}\left(\boldsymbol{x} ; \boldsymbol{\vartheta}_{1}\right)}}\left(1-\exp \left(-\left(e^{-\eta_{3}\left(\boldsymbol{x} ; \boldsymbol{\vartheta}_{3}\right)}\right) v_{2}^{* \tau}\right)\right) .
\end{aligned}
$$

Expressions for other outcome probabilities shown in Table 4.1 are given in Appendix A.2. Because $\beta_{3}$ must be positive, $\boldsymbol{\vartheta}_{3}$ must satisfy the condition that $\beta_{3}=e^{-\eta_{3}\left(\boldsymbol{x} ; \boldsymbol{\vartheta}_{3}\right)}-$ $\beta_{1}-\beta_{2}>0$ for $x \in \mathfrak{X}$.

\subsubsection{A Locally Optimal Design}

The likelihood of a single trial with two component failures $U$ and $V$ at stress $x$ is

$$
L(\boldsymbol{\theta} \mid u, v ; x)=p_{11}^{u v} \times p_{10}^{u(1-v)} \times p_{01}^{(1-u) v} \times p_{00}^{(1-u)(1-v)} .
$$

Under standard regularity conditions (cf. Ferguson (1996), Chapter 18), the information matrix of a single observation at a stress $x$ is

$$
\mu(x, \boldsymbol{\theta})=E\left\{\left[\frac{\partial}{\partial \boldsymbol{\theta}} \log L(\boldsymbol{\theta} \mid u, v ; x)\right]\left[\frac{\partial}{\partial \boldsymbol{\theta}} \log L(\boldsymbol{\theta} \mid u, v ; x)\right]^{T}\right\},
$$


and the average information matrix for design $\xi$ is

$$
M(\xi, \boldsymbol{\theta})=\sum_{i=1}^{K} w_{i} \mu\left(x_{i}, \boldsymbol{\theta}\right)
$$

Then the locally optimal design is defined as

$$
\xi^{*}=\arg \min _{\xi \in \Xi(\mathfrak{X})} \Psi(M(\xi, \boldsymbol{\theta})),
$$

where $\Xi(\mathfrak{X})$ is a set of all possible combinations of design weights and support points on $\mathfrak{X} ; \Psi(M(\xi, \boldsymbol{\theta}))=|M(\xi, \boldsymbol{\theta})|^{-1}$, and $|\cdot|$ denotes the determinant.

\subsubsection{A Penalized Locally Optimal Design}

A test of a mechanical system results in component damages and trial costs that depend on the levels of stress used. Penalty function can be used to incorporate these considerations into the design process. If components are expensive and testing is destructive, one may wish to penalize high stress levels. Similar to Dragalin and Fedorov (2005), we consider the penalty function

$$
\phi\left(x, \boldsymbol{\theta}, C_{1}, C_{2}\right)=\left(1-p_{1} \cdot(x, \boldsymbol{\theta})\right)^{-C_{1}}\left(1-p_{\cdot 1}(x, \boldsymbol{\theta})\right)^{-C_{2}}+c,
$$

where $p_{1}(x, \boldsymbol{\theta})$ and $p_{\cdot 1}(x, \boldsymbol{\theta})$ are marginal outcome probabilities, $C_{1}>0, C_{2}>0$ and $c$ is the trial cost. This penalty function increases with marginal probabilities of component failures. When $C_{1}=0$, the penalty is added only for tests at stresses with high probability of failure in component 2 , whereas, if $C_{2}=0$ the penalty is added only for tests at stresses with high probability of failure in component 1 . To 
avoid infinite penalties, we bound the penalty function so that $\phi\left(x, \boldsymbol{\theta}, C_{1}, C_{2}\right) \in[a, b]$ where $a, b \in \mathbf{R}^{+}$. For illustration, in Figure 4.1 we assume that the minimum penalty is $a=1$; the maximum penalty should not be greater than the total price of the components which is 5 . We let the trial cost be $c=0.2$. Therefore, we truncate (4.15) at 5.2 so the penalty function becomes constant when it reaches 5.2.

Let $\Phi(\xi)=\sum_{i=1}^{K} w_{i} \phi\left(x_{i}, \boldsymbol{\theta}\right)$ denote the expected penalty in design $\xi$. If the total cost is restricted, i.e., $n \Phi(\xi)<C$ where $C$ is a predetermined limit on the total "cost" of an experiment, then a penalized locally optimal design can be defined as

$$
\xi^{*}=\arg \min _{\xi \in \Xi(\mathfrak{x})} \Psi(n M(\xi, \boldsymbol{\theta}))
$$

under the condition

$$
n \Phi(\xi)<C
$$

(see Gaivoronski and Fedorov (1984), and Cook and Fedorov (1995)). If a design is continuous, design (4.16) under condition (4.17) is equivalent to

$$
\xi^{*}=\arg \min _{\xi \in \Xi(\mathfrak{X})} \Psi\left(\frac{M(\xi, \boldsymbol{\theta})}{\Phi(\xi)}\right) .
$$




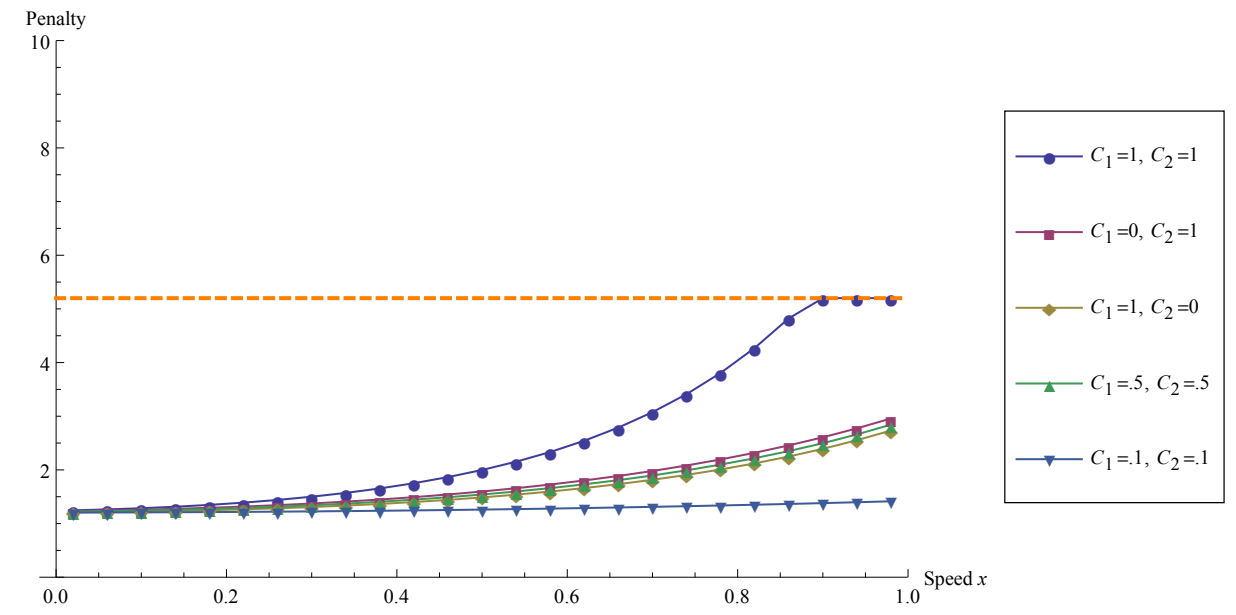

Figure 4.1: Bounded penalty functions

$\phi\left(x, \boldsymbol{\theta}, C_{1}, C_{2}\right)=\left(1-p_{1} \cdot(x, \boldsymbol{\theta})\right)^{-C_{1}}\left(1-p_{\cdot 1}(x, \boldsymbol{\theta})\right)^{-C_{2}}+c$, where $c=0.2$, $\boldsymbol{\theta}=\left(\theta_{0}=-2, \theta_{1}=4, \theta_{2}=5, \theta_{3}=2, \tau=2, \beta_{1}=0.1, \beta_{2}=0.2\right)$ 


\subsubsection{Information Matrix}

The log-likelihood function of a single system with two component failures $U$ and $V$ at stress $\boldsymbol{x}$ is

$$
\begin{aligned}
l(\boldsymbol{\theta} \mid u, v ; \boldsymbol{x})= & u v \log \left(p_{11}\right)+u(1-v) \log \left(p_{10}\right)+(1-u) v \log \left(p_{01}\right) \\
& +(1-u)(1-v) \log \left(1-p_{10}-p_{01}-p_{11}\right) .
\end{aligned}
$$

The score function can be written as the product of three matrices: $\partial l(\boldsymbol{\theta} \mid u, v ; \boldsymbol{x}) / \partial \boldsymbol{\theta}=$ $A B C$, where

$$
\begin{aligned}
& A=\left[\begin{array}{llllll}
\frac{\partial \eta_{1}\left(\boldsymbol{x} ; \boldsymbol{\vartheta}_{1}\right)}{\partial \boldsymbol{\vartheta}_{1}} & \frac{\partial \eta_{2}\left(\boldsymbol{x} ; \boldsymbol{\vartheta}_{2}\right)}{\partial \boldsymbol{\vartheta}_{2}} & \frac{\partial \eta_{3}\left(\boldsymbol{x} ; \boldsymbol{\vartheta}_{3}\right)}{\partial \boldsymbol{\vartheta}_{3}} & 1 & 1 & 1
\end{array}\right] \times \mathbf{1}_{3 \times 1} \\
& \frac{\partial \eta_{j}\left(\boldsymbol{x} ; \boldsymbol{\vartheta}_{j}\right)}{\partial \boldsymbol{\vartheta}_{j}}=\left[\begin{array}{llll}
\frac{\partial \eta_{j}\left(\boldsymbol{x} ; \boldsymbol{\vartheta}_{j}\right)}{\partial \vartheta_{j 1}} & \frac{\partial \eta_{j}\left(\boldsymbol{x} ; \boldsymbol{\vartheta}_{j}\right)}{\partial \vartheta_{j 2}} & \ldots & \frac{\partial \eta_{j}\left(\boldsymbol{x} ; \boldsymbol{\vartheta}_{j}\right)}{\partial \vartheta_{j m_{j}}}
\end{array}\right], j=1,2,3 ;
\end{aligned}
$$

$m_{j}$ is the number of parameters in $\boldsymbol{\vartheta}_{j}$ and $\mathbf{1}_{a \times b}$ is a $a \times b$ matrix of ones; 


$$
\left[\begin{array}{ccccc}
\frac{\partial p_{11}}{\partial \eta_{1}\left(\boldsymbol{x} ; \boldsymbol{\vartheta}_{1}\right)} \times \mathbf{1}_{m_{1} \times 1} & \frac{\partial p_{10}}{\partial \eta_{1}\left(\boldsymbol{x} ; \boldsymbol{\vartheta}_{1}\right)} \times \mathbf{1}_{m_{1} \times 1} & \frac{\partial p_{01}}{\partial \eta_{1}\left(\boldsymbol{x} ; \boldsymbol{\vartheta}_{1}\right)} \times \mathbf{1}_{m_{1} \times 1} \\
\frac{\partial p_{11}}{\partial \eta_{2}\left(\boldsymbol{x} ; \boldsymbol{\vartheta}_{2}\right)} \times \mathbf{1}_{m_{2} \times 1} & \frac{\partial p_{10}}{\partial \eta_{2}\left(\boldsymbol{x} ; \boldsymbol{\vartheta}_{2}\right)} \times \mathbf{1}_{m_{2} \times 1} & \frac{\partial p_{01}}{\partial \eta_{2}\left(\boldsymbol{x} ; \boldsymbol{\vartheta}_{2}\right)} \times \mathbf{1}_{m_{2} \times 1} \\
\frac{\partial p_{11}}{\partial \eta_{3}\left(\boldsymbol{x} ; \boldsymbol{\vartheta}_{3}\right)} \times \mathbf{1}_{m_{3} \times 1} \frac{\partial p_{10}}{\partial \eta_{3}\left(\boldsymbol{x} ; \boldsymbol{\vartheta}_{3}\right)} \times \mathbf{1}_{m_{3} \times 1} \frac{\partial p_{01}}{\partial \eta_{3}\left(\boldsymbol{x} ; \boldsymbol{\vartheta}_{3}\right)} \times \mathbf{1}_{m_{3} \times 1} \\
\frac{\partial p_{11}}{\partial \tau} \\
\frac{\partial p_{11}}{\partial \beta_{1}} \\
\frac{\partial p_{11}}{\partial \beta_{2}}
\end{array}\right]
$$

Thus the information matrix can be written as

$$
\begin{aligned}
\mu(x, \boldsymbol{\theta}) & =E\left[\left(\frac{\partial l(\boldsymbol{\theta} \mid u, v ; \boldsymbol{x})}{\partial \boldsymbol{\theta}}\right)\left(\frac{\partial l(\boldsymbol{\theta} \mid u, v ; \boldsymbol{x})}{\partial \boldsymbol{\theta}}\right)^{T}\right]=E\left((A B C)(A B C)^{T}\right) \\
& =E\left(A B C C^{T} B^{T} A^{T}\right)=A B\left[E\left(C C^{T}\right)\right] B^{T} A^{T}=A B D B^{T} A^{T}
\end{aligned}
$$


where $D=E\left(C C^{T}\right)$. Since $U \sim \operatorname{Bernoulli}\left(p_{1}.\right) ; V \sim \operatorname{Bernoulli}\left(p_{.1}\right)$; and $U$ and $V$ are dependent, $E\left(C C^{T}\right)$ is

$$
\left[\begin{array}{ccc}
\frac{1}{p_{11}+\frac{1}{1-p_{10}-p_{01}-p_{11}}} & \frac{1}{1-p_{10}-p_{01}-p_{11}} & \frac{1}{1-p_{10}-p_{01}-p_{11}} \\
\frac{1}{1-p_{10}-p_{01}-p_{11}} & \frac{1}{p_{10}}+\frac{1}{1-p_{10}-p_{01}-p_{11}} & \frac{1}{1-p_{10}-p_{01}-p_{11}} \\
\frac{1}{1-p_{10}-p_{01}-p_{11}} & \frac{1}{1-p_{10}-p_{01}-p_{11}} & \frac{1}{p_{01}}+\frac{1}{1-p_{10}-p_{01}-p_{11}}
\end{array}\right]
$$

\subsubsection{The Equivalence Theorem}

The Kiefer-Wolfowitz equivalence theorem (1960), which was extended to cover nonlinear functions by White (1973), is utilized to find the locally D-optimal designs. The following are equivalent (see Hackl and Fedorov (1997) for a list of equivalent conditions for other criteria):

1. $\xi^{*}(\boldsymbol{\theta})=\arg \min _{\xi}|M(\xi, \boldsymbol{\theta})|^{-1}$.

2. $\xi^{*}(\boldsymbol{\theta})=\arg \min _{\xi} \max _{x \in \mathfrak{X}} d(x, \xi, \boldsymbol{\theta})$ where $d(x, \xi, \boldsymbol{\theta})=\operatorname{tr}\left\{\mu(x, \boldsymbol{\theta}) M^{-1}(\xi, \boldsymbol{\theta})\right\}$.

3. $d\left(x, \xi^{*}, \boldsymbol{\theta}\right) \leq m$, for all $x \in \mathfrak{X}$, where $m=\operatorname{dim}(\boldsymbol{\theta})$. Moreover, $d\left(x, \xi^{*}, \boldsymbol{\theta}\right)=m$ at all support points of $\xi^{*}$.

If the penalty function is included in an experiment, the following are equivalent:

1. $\xi^{*}(\boldsymbol{\theta})=\arg \min _{\xi}|M(\xi, \boldsymbol{\theta}) / \Phi(\xi, \boldsymbol{\theta})|^{-1}$. 
2. $\xi^{*}(\boldsymbol{\theta})=\arg \min _{\xi} \max _{x \in \mathfrak{X}}\{d(x, \xi, \boldsymbol{\theta})-(\phi(x, \boldsymbol{\theta}) / \Phi(\xi, \boldsymbol{\theta})) m\}$

where $d(x, \xi, \boldsymbol{\theta})=\operatorname{tr}\left\{\mu(x, \boldsymbol{\theta}) M^{-1}(\xi, \boldsymbol{\theta})\right\}$.

3. $\phi^{-1}(x, \boldsymbol{\theta}) d\left(x, \xi^{*}, \boldsymbol{\theta}\right) \leq \Phi\left(\xi^{*}, \boldsymbol{\theta}\right) m$, for all $x \in \mathfrak{X}$, where $m=\operatorname{dim}(\boldsymbol{\theta})$.

Moreover, $\phi^{-1}(x, \boldsymbol{\theta}) d\left(x, \xi^{*}, \boldsymbol{\theta}\right)=\Phi\left(\xi^{*}, \boldsymbol{\theta}\right) m$ at all support points of the optimal design.

\subsection{A Crash Test with Two Components: An Ex- ample}

Suppose there is a system of two components. Extreme forces or stresses can cause one or two components failure or damage. So there are four possible failures given a stress (Table 4.1). A crash test is destructive testing that is used to ensure safety for various models of cars or vehicles or related systems and components. Major providers are the National Highway Traffic Safety Administration (NHTSA), the Insurance Institute for Highway Safety (IIHS), and the Australasian New Car Assessment Program (ANCAP). A problem is that the crash test is usually performed

on one or a few of vehicles under one set of conditions. For example, the ANCAP conducts the frontal offset crash test with one speed level $(64 \mathrm{~km} / \mathrm{h})$. For more details, we recommend ANCAP's web page (www.ancap.com.au). It is unreliable to base safety assessments on one vehicle operating at one speed because there are several sources of variation. To ameliorate these problems, a good statistical model for various speeds can be estimated with a small number of trials to obtain a significantly better assessment. 


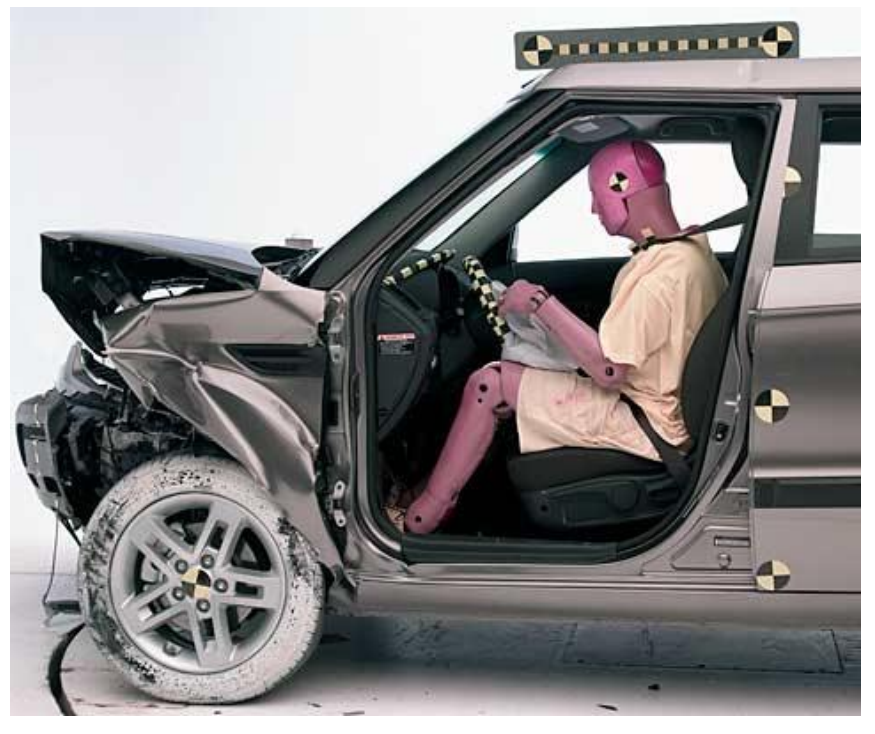

Figure 4.2: IIHS frontal offset crash test 
Two key components are considered in this chapter. Let component 1 be the human component (human dummy), and let component 2 be the machine component (vehicle). We assume the amount of damage to the two components follows the bivariate Weibull density with only one covariate which is standardized speed: $x=$ $\frac{\text { Observed speed - Minimum speed }}{\text { Maximum speed - Minimum speed }}$. We fix the "maximum speed" at 100 miles/hour, and the "minimum speed" at 1 mile/hour. Then the amounts of damage are dichotomized by (4.11). Predetermined thresholds of fatal damage for the two components are assumed to be $v_{1}^{*}$ and $v_{2}^{*}$, such as critical cracks in a human dummy's neck bone (e.g., that might have been fatal to a human) and unrepairable damage to the front bumper, respectively. We set $v_{1}^{*}=.8$, and $v_{2}^{*}=.7$. Let $\eta_{i}\left(\boldsymbol{x} ; \boldsymbol{\vartheta}_{i}\right)=\theta_{0}+\theta_{i} x$, where $i=1,2,3$. Then the predictor functions are

$$
\begin{aligned}
& -\log \left(\beta_{5}+\beta_{3}\right)=\theta_{0}+\theta_{1} x \\
& -\log \left(\beta_{4}+\beta_{3}\right)=\theta_{0}+\theta_{2} x \\
& -\log \left(\beta_{1}+\beta_{2}+\beta_{3}\right)=\theta_{0}+\theta_{3} x .
\end{aligned}
$$

When $x=0$, the two components don't get any damage which justifies the identical intercepts $\left(\theta_{0}\right)$. Figures 4.3 shows selected conditional marginal density functions of the amount of damage for component 1 given standardized speeds. As is desired, at the higher speeds, there is greater chance of serious damages; at the lower speeds, there is more chance of slight damages. The conditional marginal density functions of the amount of damage for component 2 given standardized speeds are similar. 


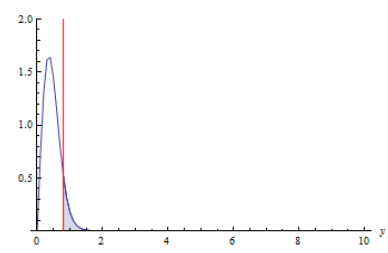

(a) $f(y \mid x=.3)$

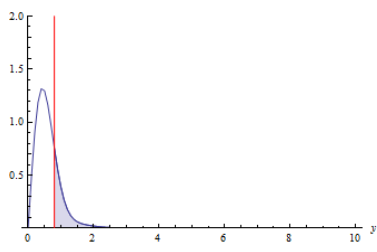

(b) $f(y \mid x=.5)$

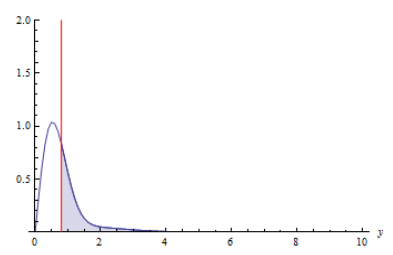

(c) $f(y \mid x=.7)$

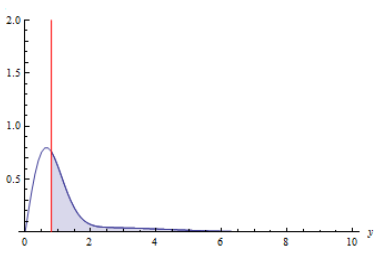

(d) $f(y \mid x=.9)$

Figure 4.3: Marginal Weibull density conditional on stress level for the component 1 . $\left(v_{1}^{*}=.8\right)$ $\theta_{0}=-2, \theta_{1}=4, \theta_{2}=5, \theta_{3}=2, \tau=2, \beta_{1}=0.1, \beta_{2}=0.2$

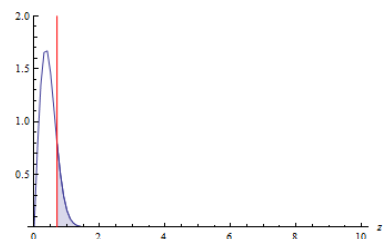

(a) $f(z \mid x=.3)$

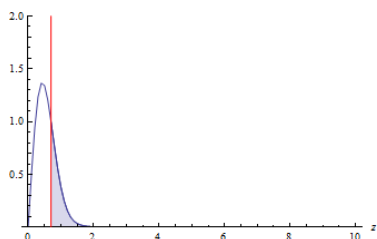

(b) $f(z \mid x=.5)$

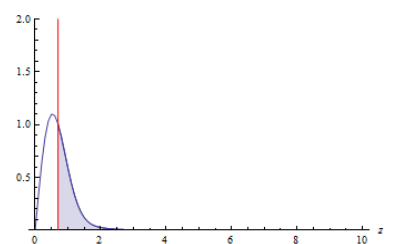

(c) $f(z \mid x=.7)$

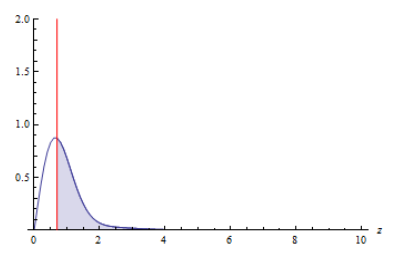

(d) $f(z \mid x=.9)$

Figure 4.4: Marginal Weibull density conditional on stress level for the component 2. $\left(v_{2}^{*}=.7\right)$

$$
\theta_{0}=-2, \theta_{1}=4, \theta_{2}=5, \theta_{3}=2, \tau=2, \beta_{1}=0.1, \beta_{2}=0.2
$$


Inserting the predictor functions (4.20) into (4.12) yields

$$
\begin{aligned}
p_{00}(x \mid \boldsymbol{\theta})= & \frac{\beta_{1}}{e^{-\left(\theta_{0}+\theta_{3} x\right)}-e^{-\left(\theta_{0}+\theta_{1} x\right)}}\left(1-\exp \left(-\left(e^{-\left(\theta_{0}+\theta_{1} x\right)}\right) v_{2}^{* \tau}\right)\right) \\
& -\frac{\beta_{2}}{e^{-\left(\theta_{0}+\theta_{3} x\right)}-e^{-\left(\theta_{0}+\theta_{2} x\right)}}\left(1-\exp \left(-\left(e^{-\left(\theta_{0}+\theta_{3} x\right)}-e^{-\left(\theta_{0}+\theta_{2} x\right)}\right) v_{2}^{* \tau}\right)\right) \\
& \times \exp \left(-\left(e^{-\left(\theta_{0}+\theta_{2} x\right)}\right) v_{1}^{* \tau}\right) \\
& +\frac{e^{-\left(\theta_{0}+\theta_{3} x\right)}-e^{-\left(\theta_{0}+\theta_{1} x\right)}-\beta_{1}}{e^{-\left(\theta_{0}+\theta_{3} x\right)}-e^{-\left(\theta_{0}+\theta_{1} x\right)}}\left(1-\exp \left(-\left(e^{-\left(\theta_{0}+\theta_{3} x\right)}\right) v_{2}^{* \tau}\right)\right) .
\end{aligned}
$$

Expressions for other outcome probabilities with the predictor functions (4.20) are given in Appendix A.3.

Replacing scale parameters $\left[\left(\beta_{5}+\beta_{3}\right),\left(\beta_{4}+\beta_{3}\right)\right.$, and $\left.\left(\beta_{1}+\beta_{2}+\beta_{3}\right)\right]$ of Weibull density (4.9) with the predictor functions : $e^{-\left(\theta_{0}+\theta_{1} x\right)}, e^{-\left(\theta_{0}+\theta_{2} x\right)}$, and $e^{-\left(\theta_{0}+\theta_{3} x\right)}$, respectively, $\beta_{3}, \beta_{4}$, and $\beta_{5}$ are eliminated from the expression of the outcome probabilities; but $\beta_{1}$, and $\beta_{2}$ remain (see Appendix A.3). This implies that $\theta_{0}$ and $\theta_{3}$ must satisfy the condition $\beta_{3}=e^{-\left(\theta_{0}+\theta_{3} x\right)}-\beta_{1}-\beta_{2}>0$ for $x \in \mathfrak{X}$. To make $p_{00}(x \mid \boldsymbol{\theta})$ a decreasing probability function and $p_{11}(x \mid \boldsymbol{\theta})$ an increasing probability function as $x$ increases, $\theta_{1}, \theta_{2}$, and $\theta_{3}$ must be positive. Summarizing the above conditions for $x \in[0,1]$, we 
have

$$
\begin{aligned}
-\infty & <\theta_{0}<-\log \left(\beta_{1}+\beta_{2}\right)-\theta_{3}, \\
0 & <\theta_{1}<\infty \\
0 & <\theta_{2}<\infty \\
0 & <\theta_{3}<-\log \left(\beta_{1}+\beta_{2}\right)-\theta_{0}, \\
0 & <\tau<\infty \\
0 & <\beta_{1}<e^{-\left(\theta_{0}+\theta_{3}\right)}-\beta_{2} \\
0 & <\beta_{2}<e^{-\left(\theta_{0}+\theta_{3}\right)}-\beta_{1} .
\end{aligned}
$$

A plot of the marginal probabilities as a function of speed is shown in Figure 4.5 assuming the parameter values $\theta_{0}=-2, \theta_{1}=4, \theta_{2}=5, \theta_{3}=2, \tau=2, \beta_{1}=0.1$, and $\beta_{2}=0.2$. The probabilities $p_{00}=\mathrm{P}$ (No failure for both components $), p_{0}=\mathrm{P}($ No failure for the component 1$)$ and $p_{\cdot 0}=\mathrm{P}($ No failure for the component 2$)$ all tend to decrease as $x$ (speed) increases; $p_{11}=\mathrm{P}$ (Failures for both components) tends to increase as $x$ (speed) increases.

Let $\boldsymbol{\theta}=\left\{\theta_{0}, \theta_{1}, \theta_{2}, \theta_{3}, \tau, \beta_{1}, \beta_{2}\right\}$. Assuming simple linear predictors with $\eta_{i}\left(\boldsymbol{x} ; \boldsymbol{\vartheta}_{i}\right)=$ $\theta_{0}+\theta_{i} x, i=1,2,3$, the information matrix (4.19) becomes $I(\boldsymbol{\theta})=G D G^{T}$ where $G=A B$ and

$$
G^{T}=\left[\begin{array}{lllllll}
\frac{\partial p_{11}}{\partial \theta_{0}} & \frac{\partial p_{11}}{\partial \theta_{1}} & \frac{\partial p_{11}}{\partial \theta_{2}} & \frac{\partial p_{11}}{\partial \theta_{3}} & \frac{\partial p_{11}}{\partial \tau} & \frac{\partial p_{11}}{\partial \beta_{1}} & \frac{\partial p_{11}}{\partial \beta_{2}} \\
\frac{\partial p_{10}}{\partial \theta_{0}} & \frac{\partial p_{10}}{\partial \theta_{1}} & \frac{\partial p_{10}}{\partial \theta_{2}} & \frac{\partial p_{10}}{\partial \theta_{3}} & \frac{\partial p_{10}}{\partial \tau} & \frac{\partial p_{10}}{\partial \beta_{1}} & \frac{\partial p_{10}}{\partial \beta_{2}} \\
\frac{\partial p_{01}}{\partial \theta_{0}} & \frac{\partial p_{01}}{\partial \theta_{1}} & \frac{\partial p_{01}}{\partial \theta_{2}} & \frac{\partial p_{01}}{\partial \theta_{3}} & \frac{\partial p_{01}}{\partial \tau} & \frac{\partial p_{01}}{\partial \beta_{1}} & \frac{\partial p_{01}}{\partial \beta_{2}}
\end{array}\right] .
$$

The partial derivatives of $I(\boldsymbol{\theta})=G D G^{T}$, i.e., of $p_{11}, p_{10}$, and $p_{01}$ with respect to $\boldsymbol{\theta}$ can be easily computed using Mathematica (Wolfram Research, Inc.(2012)). 


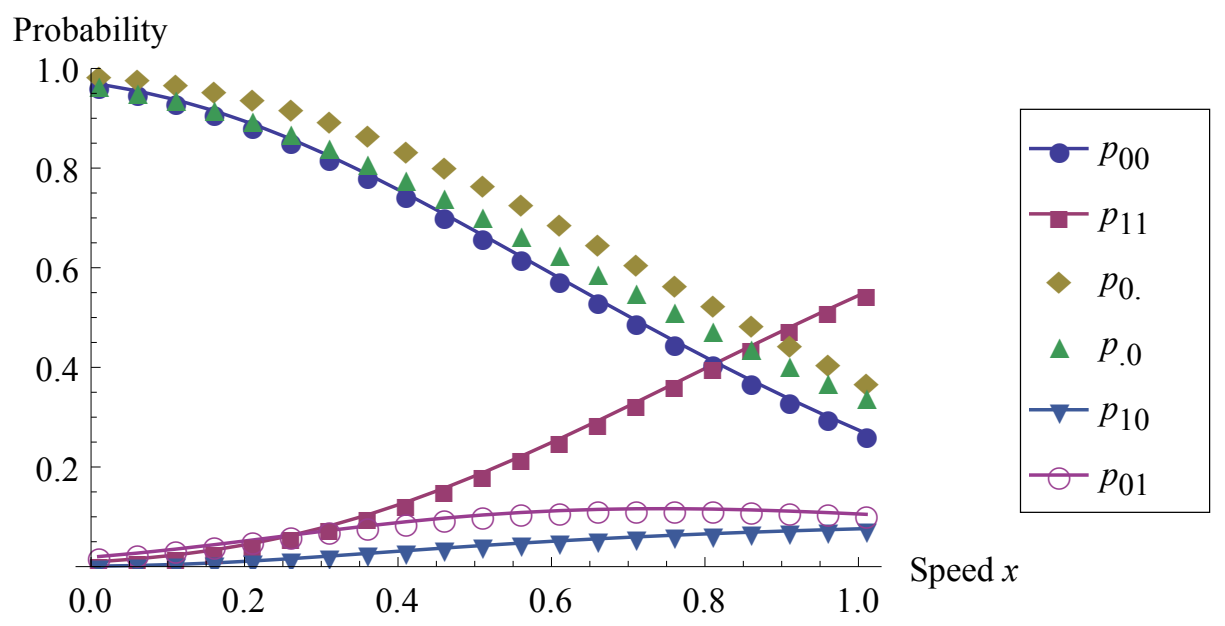

Figure 4.5: Outcome and marginal probabilities $\theta_{0}=-2, \theta_{1}=4, \theta_{2}=5, \theta_{3}=2, \tau=2, \beta_{1}=0.1, \beta_{2}=0.2$ 
To find locally D-optimal designs and penalized D-optimal designs, the directional derivative in the exchange algorithm is used (cf. Fedorov and Hackl (1997), Chapter 3). Simple steps for the exchange algorithm are listed in Appendix A.5. The average information matrix (4.14) must be full rank to estimate all model parameters, and the average information matrix (4.14) with the linear predictor functions (4.20) requires at least three design points to be full rank. Therefore, all locally optimal designs have at least three design points.

The bars in Figures 4.6, 4.7, 4.8, and 4.9 represent the proportion of trials allocated to specific speeds. The penalized D-optimal designs in Figures 4.7, 4.8, and 4.9 used the penalty function (4.15) with $\left(C_{1}=1, C_{2}=1\right),\left(C_{1}=0, C_{2}=1\right)$, and $\left(C_{1}=1, C_{2}=0\right)$, respectively. Since there is greater penalty when either or both component failure probabilities are higher, there are fewer allocations at higher speeds in the penalized D-optimal designs. Compared to the penalized D-optimal designs, the locally D-optimal design has more information. The loss of information caused by penalizing stresses with high failure rates can be found in Table 4.2 by comparing information from the locally optimal design.

\begin{tabular}{|c|c|c|}
\hline$\frac{\left|M\left(\xi_{P D_{1}}, \boldsymbol{\theta}\right)\right|^{1 / m}}{\left|M\left(\xi_{D}, \boldsymbol{\theta}\right)\right|^{1 / m}}$ & $\frac{\left|M\left(\xi_{P D_{2}}, \boldsymbol{\theta}\right)\right|^{1 / m}}{\left|M\left(\xi_{D}, \boldsymbol{\theta}\right)\right|^{1 / m}}$ & $\frac{\left|M\left(\xi_{P D_{3}}, \boldsymbol{\theta}\right)\right|^{1 / m}}{\left|M\left(\xi_{D}, \boldsymbol{\theta}\right)\right|^{1 / m}}$ \\
\hline \hline .8089 & .9108 & .9172 \\
\hline
\end{tabular}

Table 4.2: Efficiency of penalized designs 


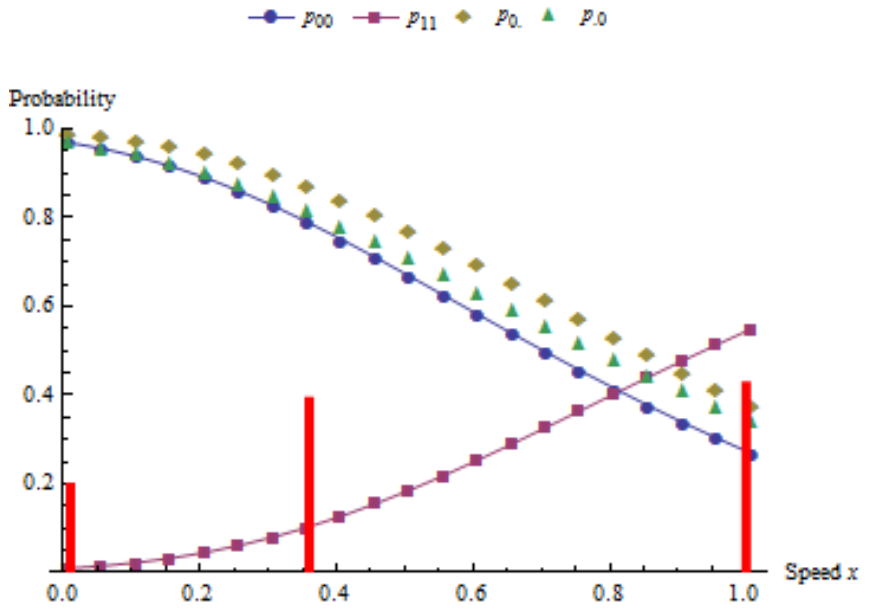

(a) Optimal design $\left(\xi_{D}\right)$

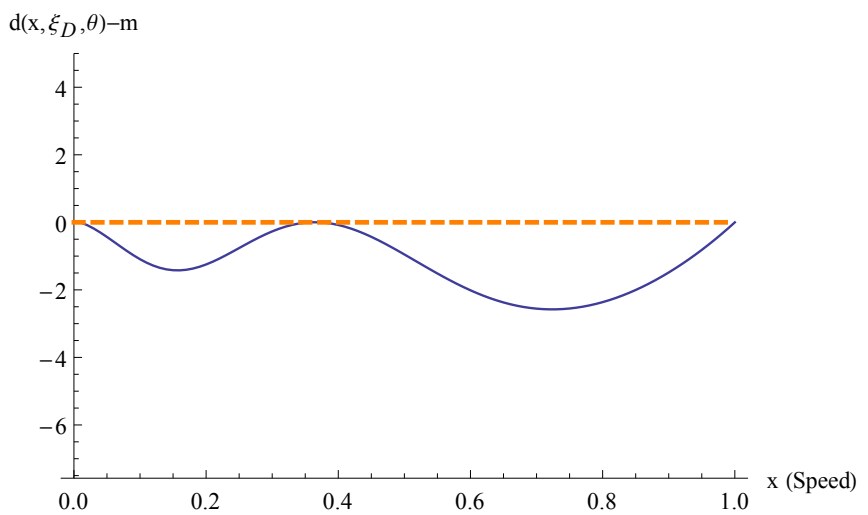

(b) $d\left(x, \xi_{D}, \boldsymbol{\theta}\right)-m$

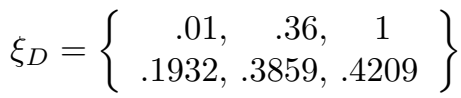

$$
\begin{aligned}
& \left|M\left(\xi_{D}, \boldsymbol{\theta}\right)\right|^{1 / m}=.0157, \\
& \theta_{0}=-2, \theta_{1}=4, \theta_{2}=5, \theta_{3}=2, \tau=2, \beta_{1}=0.1, \beta_{2}=0.2
\end{aligned}
$$

Figure 4.6: The locally D-optimal design with a linear predictor under the bivariate Weibull distribution.

**The candidate set contains 100 equally spaced points within $(0,1]$. The bars represent allocation proportions to speeds for the locally D-optimal design. 

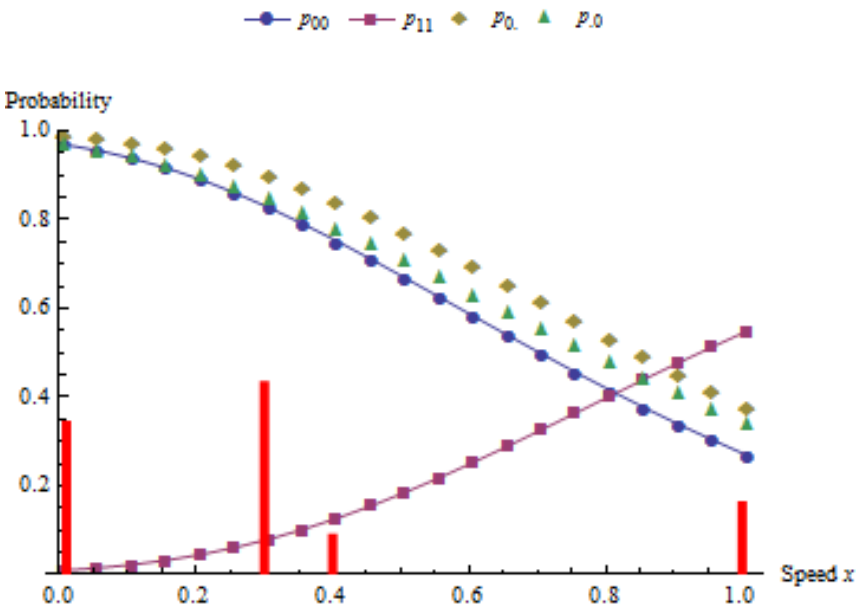

(a) Penalized D-optimal design with $C_{1}=1, C_{2}=1\left(\xi_{P D 1}\right)$

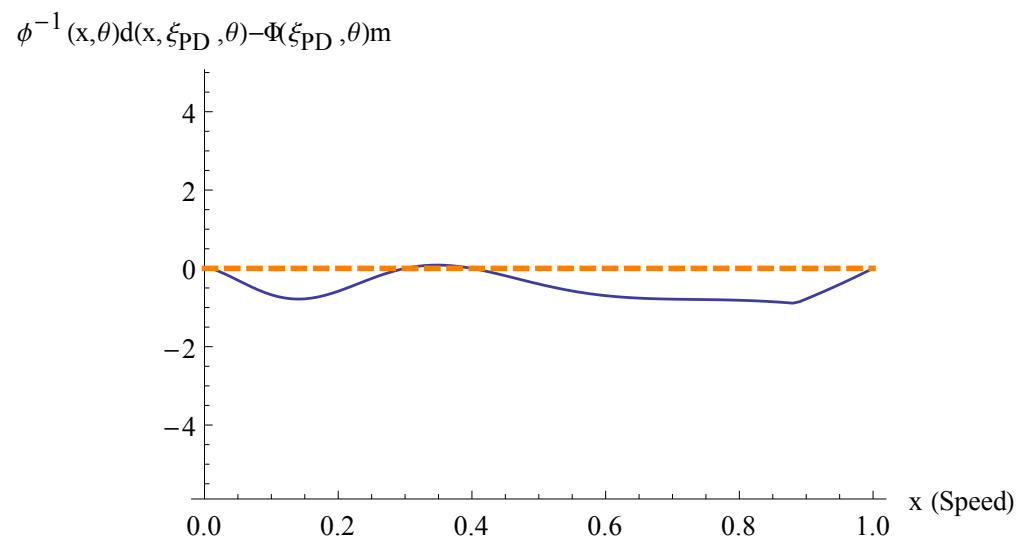

(b) $\left|\phi^{-1}(x, \boldsymbol{\theta}) d\left(x, \xi_{P D 1}, \boldsymbol{\theta}\right)-\Phi^{-1}\left(\xi_{P D 1}, \boldsymbol{\theta}\right) m\right|$

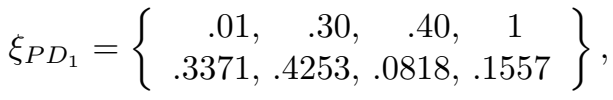

$$
\begin{aligned}
& \left|M\left(\xi_{P D_{1}}, \boldsymbol{\theta}\right)\right|^{1 / m}=.0127, \\
& \theta_{0}=-2, \theta_{1}=4, \theta_{2}=5, \theta_{3}=2, \tau=2, \beta_{1}=0.1, \beta_{2}=0.2 \text {, } \\
& \phi(x, \boldsymbol{\theta})=\left(1-p_{1} \cdot(x, \boldsymbol{\theta})\right)^{-C_{1}}\left(1-p_{\cdot 1}(x, \boldsymbol{\theta})\right)^{-C_{2}}+0.2 .
\end{aligned}
$$

Figure 4.7: The penalized D-optimal design $\left(C_{1}=1, C_{2}=1\right)$ with a linear predictor under the bivariate Weibull distribution.

**The candidate set contains 100 equally spaced points within $(0,1]$. The bars represent allocation proportions to speeds for the penalized D-optimal design. 

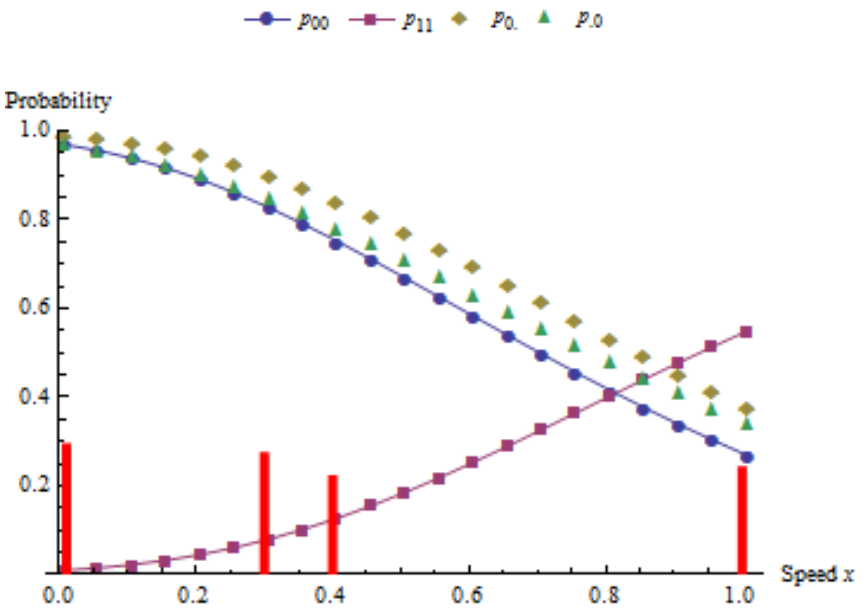

(a) Penalized D-optimal design with $C_{1}=0, C_{2}=1\left(\xi_{P D 2}\right)$

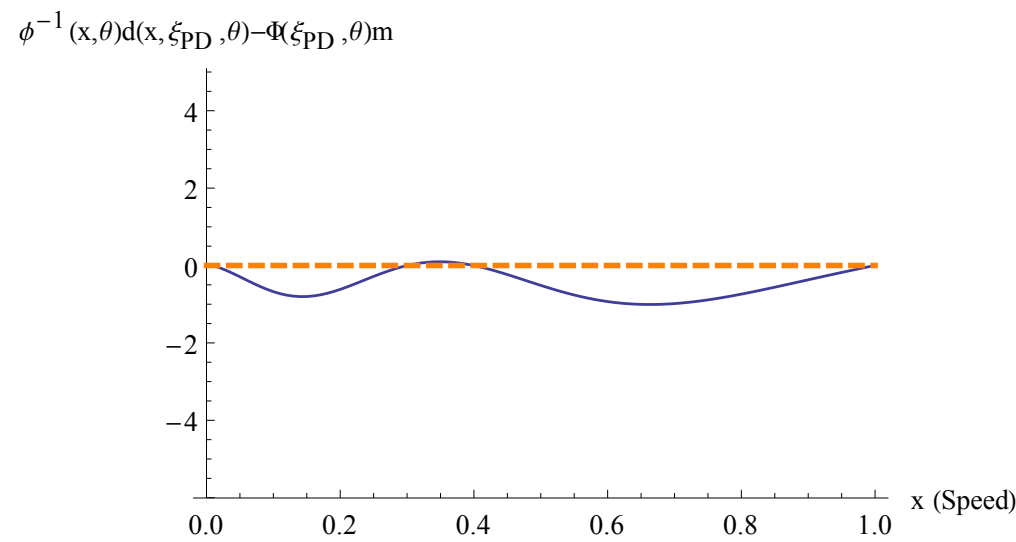

(b) $\left|\phi^{-1}(x, \boldsymbol{\theta}) d\left(x, \xi_{P D 2}, \boldsymbol{\theta}\right)-\Phi^{-1}\left(\xi_{P D 2}, \boldsymbol{\theta}\right) m\right|$

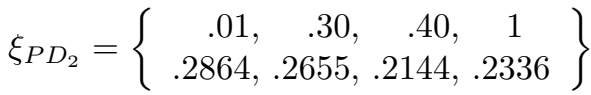

$$
\begin{aligned}
& \left|M\left(\xi_{P D_{2}}, \boldsymbol{\theta}\right)\right|^{1 / m}=.0143, \\
& \theta_{0}=-2, \theta_{1}=4, \theta_{2}=5, \theta_{3}=2, \tau=2, \beta_{1}=0.1, \beta_{2}=0.2 \text {, } \\
& \phi(x, \boldsymbol{\theta})=\left(1-p_{1} \cdot(x, \boldsymbol{\theta})\right)^{-C_{1}}\left(1-p_{\cdot 1}(x, \boldsymbol{\theta})\right)^{-C_{2}}+0.2 \text {. }
\end{aligned}
$$

Figure 4.8: The penalized D-optimal design $\left(C_{1}=0, C_{2}=1\right)$ with a linear predictor under the bivariate Weibull distribution.

**The candidate set contains 100 equally spaced points within $(0,1]$. The bars represent allocation proportions to speeds for the penalized D-optimal design. 

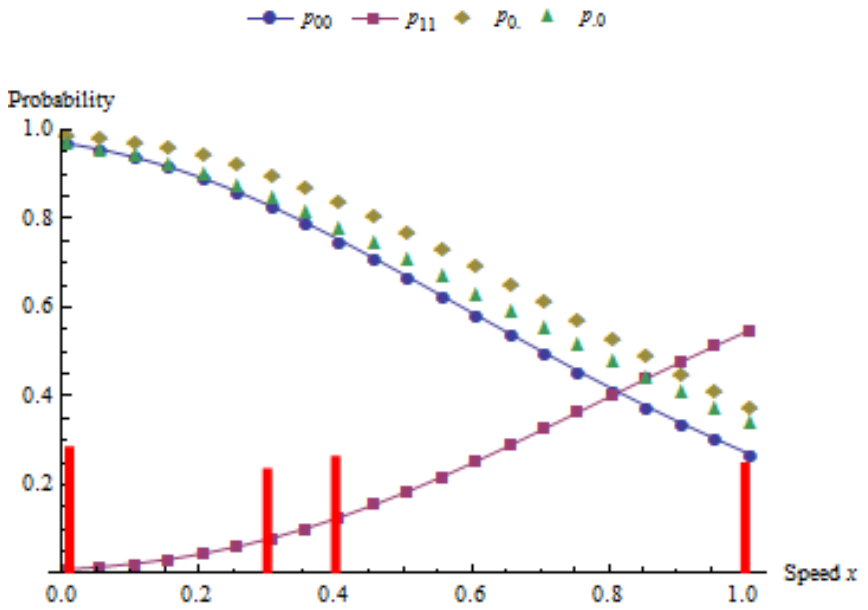

(a) Penalized D-optimal design with $C_{1}=1, C_{2}=0\left(\xi_{P D 3}\right)$

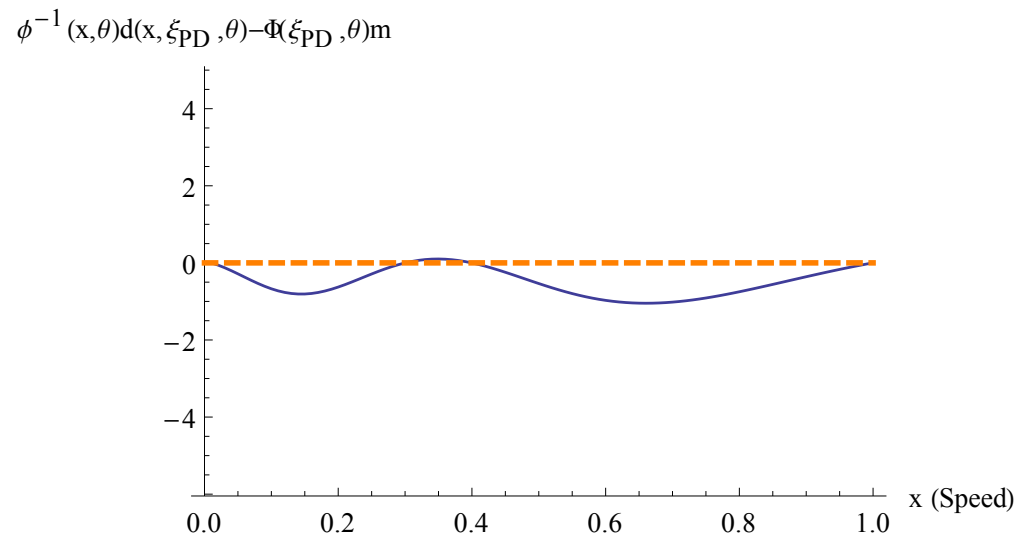

(b) $\left|\phi^{-1}(x, \boldsymbol{\theta}) d\left(x, \xi_{P D 3}, \boldsymbol{\theta}\right)-\Phi^{-1}\left(\xi_{P D 3}, \boldsymbol{\theta}\right) m\right|$

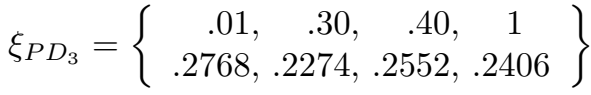

$$
\begin{aligned}
& \left|M\left(\xi_{P D_{3}}, \boldsymbol{\theta}\right)\right|^{1 / m}=.0144, \\
& \theta_{0}=-2, \theta_{1}=4, \theta_{2}=5, \theta_{3}=2, \tau=2, \beta_{1}=0.1, \beta_{2}=0.2 \text {, } \\
& \phi(x, \boldsymbol{\theta})=\left(1-p_{1} \cdot(x, \boldsymbol{\theta})\right)^{-C_{1}}\left(1-p_{\cdot 1}(x, \boldsymbol{\theta})\right)^{-C_{2}}+0.2 \text {. }
\end{aligned}
$$

Figure 4.9: The penalized D-optimal design $\left(C_{1}=1, C_{2}=0\right)$ with a linear predictor under the bivariate Weibull distribution.

**The candidate set contains 100 equally spaced points within $(0,1]$. The bars represent allocation proportions to speeds for the penalized D-optimal design. 


\subsection{Robustness}

Unlike optimal designs with linear models, optimal designs for nonlinear response models depend on parameter values because the information matrix contains the parameters. In this section, we study the robustness of locally optimal designs to model misspecification. In the next section, we show that a design can be substantially improved by splitting the total sample into groups and running a series of experiments that learn from prior results. Table 4.3 shows the parameter vector values used to evaluate robustness. They cover a wide range of the possible values. The outcome probability changes for different choices of the model parameters in Table 4.3 are shown in Figures A.2-A.8 in Appendix A.7. Figures A.3-A.4 in Appendix A.7 show that varying $\theta_{1}$ and $\theta_{2}$, respectively, has little effect on $\left\{p_{00}, p_{11}, p_{0 .}, p_{.0}\right\}$. The $\left\{p_{00}, p_{11}, p_{0 .}, p_{.0}\right\}$ are almost same with respect to $\theta_{1}$ and $\theta_{2}$ because $\theta_{1}$ and $\theta_{2}$ only appear in the marginal density functions of $Z$ and $Y$, respectively; other parameters appear in both marginal density functions.

Table 4.4 contains the efficiency of D-optimal designs corresponding to parameter vector values $\boldsymbol{\theta}$ in the Table 4.3. The entry at the $i$ th row and $j$ th column of the table is the ratio of the determinant of information matrices:

$$
\text { Efficiency }=\left(\frac{\left|M\left(\xi_{\boldsymbol{\theta}_{j}}, \boldsymbol{\theta}_{i}\right)\right|}{\left|M\left(\xi_{\boldsymbol{\theta}_{i}}, \boldsymbol{\theta}_{i}\right)\right|}\right)^{1 / m}
$$

where $m$ is the number of parameters, and $i$ and $j=1,2, \ldots, 16$. Remarkably for such a complex model, for a wide range of parameter values, the locally optimal designs have high efficiency. Most of cases evaluated have at least 90\% efficiency. However, design efficiency suffered when $\theta_{0}$ was misspecified. For example, assuming $\boldsymbol{\theta}_{2}$ is a 
true parameter, the lowest efficiency value $(.27)$ in the second row and sixteenth column tells us that if the true parameter $\boldsymbol{\theta}_{2}$ is misspecified as $\boldsymbol{\theta}_{16}, 3.7(1 / .27=3.70)$ times as many samples are needed to produce the same accuracy of the parameter estimates using $\xi_{\boldsymbol{\theta}_{16}}$ instead of $\xi_{\boldsymbol{\theta}_{2}}$. 


\begin{tabular}{|c|c|c|c|}
\hline $\boldsymbol{\theta}_{i}$ & $\theta_{0}, \theta_{1}, \theta_{2}, \theta_{3}, \tau, \beta_{1}, \beta_{2}$ & & Locally optimal design \\
\hline $\boldsymbol{\theta}_{1}$ (Figure 4.5) & $-2,4,5,2,2,0.1,0.2$ & $\xi_{\boldsymbol{\theta}_{1}}=\{$ & $\left.\begin{array}{rrc}.01, & .36, & 1 \\
.1932, & .3859, & .4209\end{array}\right\}$ \\
\hline $\boldsymbol{\theta}_{2}$ (Figure A.2-(a)) & $-3,4,5,2,2,0.1,0.2$ & $\xi_{\boldsymbol{\theta}_{2}}=\{$ & $\begin{array}{rcc}.17, & .51, & 1 \\
.1534, & .4187, & .4279\end{array}$ \\
\hline $\boldsymbol{\theta}_{3}$ (Figure A.2-(c)) & $-1,4,5,2,2,0.1,0.2$ & $\xi_{\boldsymbol{\theta}_{3}}=\{$ & $\begin{array}{ccc}.01, & .30, & 1 \\
.2537, & .3512, & .3951\end{array}$ \\
\hline $\boldsymbol{\theta}_{4}$ (Figure A.3-(a)) & $-2,3,5,2,2,0.1,0.2$ & $\xi_{\boldsymbol{\theta}_{4}}=\{$ & $\begin{array}{cccc}.01, & .39, & .40, & 1 \\
.1972, & .2679, & .1159, & .4189 \\
\end{array}$ \\
\hline $\boldsymbol{\theta}_{5}$ (Figure A.3-(c)) & $-2,100,5,2,2,0.1,0.2$ & $\xi_{\boldsymbol{\theta}_{5}}=\{$ & $\left.\begin{array}{rrc}.02, & .35, & 1 \\
.1936, & .3849, & .4215\end{array}\right\}$ \\
\hline $\boldsymbol{\theta}_{6}$ (Figure A.4-(a)) & $-2,4,4,2,2,0.1,0.2$ & $\xi_{\boldsymbol{\theta}_{6}}=\{$ & $\begin{array}{rrrrc}.01, & .39, & .40, & .41, & 1 \\
.1985, & .0771, & .2806, & .0258, & .4180\end{array}$ \\
\hline $\boldsymbol{\theta}_{7}($ Figure A.4-(c)) & $-2,4,100,2,2,0.1,0.2$ & $\xi_{\boldsymbol{\theta}_{7}}=\{$ & $\left.\begin{array}{rrrr}.02, & .41, & .42, & 1 \\
.3709, & .1464, .1057, & .3770\end{array}\right\}$ \\
\hline $\boldsymbol{\theta}_{8}$ (Figure A.5-(a)) & $-2,4,5,1,2,0.1,0.2$ & $\xi_{\boldsymbol{\theta}_{8}}=$ & $\left.\begin{array}{cccc}.01, & .35, & .36, & 1 \\
.2073, & .3177, & .0517, & .4233\end{array}\right\}$ \\
\hline $\boldsymbol{\theta}_{9}$ (Figure A.5-(c)) & $-2,4,5,3,2,0.1,0.2$ & $\xi_{\boldsymbol{\theta}_{9}}=$ & $\begin{array}{rrrrr}.03, & .37, & .38, & .39, & 1 \\
.1987, & .0114, & .2103, & .1689, & .4106\end{array}$ \\
\hline $\boldsymbol{\theta}_{10}($ Figure A.6-(a)) & $-2,4,5,2,1,0.1,0.2$ & $\xi_{\boldsymbol{\theta}_{10}}=$ & $\begin{array}{rcc}.06, & .40, & 1 \\
.1845, & .3917, & .4238\end{array}$ \\
\hline $\boldsymbol{\theta}_{11}$ (Figure A.6-(c)) & $-2,4,5,2,3,0.1,0.2$ & $\xi_{\boldsymbol{\theta}_{11}}=$ & $\begin{array}{ccc}.01, & .34, & 1 \\
.2105, & .3728, & .4166\end{array}$ \\
\hline $\boldsymbol{\theta}_{12}($ Figure A.7-(a)) & $-2,4,5,2,2,0.05,0.2$ & $\xi_{\boldsymbol{\theta}_{12}}=$ & $\begin{array}{cccc}.01, & .36, & .37, & 1 \\
.1934, & .2920, & .0939, .4207 \\
\end{array}$ \\
\hline $\boldsymbol{\theta}_{13}$ (Figure A.7-(c)) & $-2,4,5,2,2,0.7,0.2$ & $\xi_{\boldsymbol{\theta}_{13}}=$ & $\left.\begin{array}{rrc}.01, & .36, & 1 \\
.1928, & .3862, & .4210\end{array}\right\}$ \\
\hline $\boldsymbol{\theta}_{14}$ (Figure A.8-(a)) & $-2,4,5,2,2,0.1,0.1$ & $\xi_{\boldsymbol{\theta}_{14}}=$ & $\left.\begin{array}{rrr}.01, & .36, & 1 \\
.1917, & .3871, .4212\end{array}\right\}$ \\
\hline $\boldsymbol{\theta}_{15}$ (Figure A.8-(c)) & $-2,4,5,2,2,0.1,0.8$ & $\xi_{\boldsymbol{\theta}_{15}}=$ & $\begin{array}{cccc}.01, & .38, & .39, & 1 \\
.2030, & .1573, & .2229, & .4169 \\
\end{array}$ \\
\hline $\boldsymbol{\theta}_{16}$ (Figure $\left.4.10-(\mathrm{b})\right)$ & $-1.5,8,10,1.5,1,0.3,0.4$ & $\xi_{\boldsymbol{\theta}_{16}}=$ & $\begin{array}{ccc}.01, & .18, & 1 \\
.2083, & .3671, & .4246 \\
\end{array}$ \\
\hline
\end{tabular}

**Among the many combinations of parameter values, for simplicity, each parameter values has been manipulated from $\boldsymbol{\theta}_{1}$. The boxes in the second column indicate the manipulated parameter values. $\boldsymbol{\theta}_{16}$ contains entirely different parameter values from $\boldsymbol{\theta}_{1}$.

Table 4.3: True parameter values for evaluation of robustness 


\begin{tabular}{|c|c|c|c|c|c|c|c|c|c|c|c|c|c|c|c|c|}
\hline${\overline{\boldsymbol{\theta}_{i}}}^{\xi_{\boldsymbol{\theta}_{i}}}$ & 1 & 2 & 3 & 4 & 5 & 6 & 7 & 8 & 9 & 10 & 11 & 12 & 13 & 14 & 15 & 16 \\
\hline$\overline{1}$ & & $*$ & & & & & & & & & & & & & & $* *$ \\
\hline 2 & $* *$ & & $\dagger \dagger$ & $* *$ & $* *$ & $* *$ & $* *$ & $\dagger \dagger$ & $* *$ & $*$ & $\dagger \dagger$ & $* *$ & $* *$ & $* *$ & $* *$ & $+\dagger$ \\
\hline 3 & & $\dagger \dagger$ & & & & & & & & $*$ & & & & & & \\
\hline 4 & & & & & & & & & & & & & & & & $* *$ \\
\hline 5 & & $\dagger \dagger$ & & & & & & & $*$ & $\dagger \dagger$ & & & & & & $* *$ \\
\hline 6 & & $*$ & & & & & & & & & & & & & & $* *$ \\
\hline 7 & $*$ & $\dagger \dagger$ & $*$ & $*$ & & $*$ & & $*$ & $*$ & $\dagger \dagger$ & $*$ & $*$ & $*$ & $*$ & $*$ & $* *$ \\
\hline 8 & & $* *$ & & & & & & & & & & & & & & $* *$ \\
\hline 9 & & $*$ & & & & & & & & & & & & & & $* *$ \\
\hline 10 & & * & & & & & & & & & & & & & & $\dagger \dagger$ \\
\hline 11 & & $* *$ & & & & & & & & & & & & & & $*$ \\
\hline 12 & & $*$ & & & & & & & & & & & & & & $* *$ \\
\hline 13 & & $*$ & & & & & & & & & & & & & & $* *$ \\
\hline 14 & & $*$ & & & & & & & & & & & & & & $* *$ \\
\hline 15 & & $*$ & & & & & & & & & & & & & & ** \\
\hline 16 & $\dagger \dagger$ & $\dagger \dagger$ & $*$ & $\dagger \dagger$ & $* *$ & $\dagger \dagger$ & $\dagger \dagger$ & $* *$ & $\dagger \dagger$ & $\dagger \dagger$ & ** & $\dagger \dagger$ & $\dagger \dagger$ & $\dagger \dagger$ & $+\dagger$ & \\
\hline
\end{tabular}

Blank : .90 $<$ Efficiency $\leq 1.00$,

$*: .80<$ Efficiency $\leq .90$,

$* *: .70<$ Efficiency $\leq .80$,

$\dagger \dagger:$ Efficiency $\leq .70$.

Table 4.4: Efficiency of locally D-optimal designs under alternative parameter values 


\subsection{An Adaptive Optimal Design}

To compensate for the initial misspecification of parameters, several authors (including Box and Hunter (1965), Fedorov (1972) and White (1975)) have proposed sequentially estimating optimal designs. Ford and Silvey (1980) proved that the design measure of this sequential procedure for a simple nonlinear model converges to the optimal measure. Atkinson, Donev, and Tobias (2007, Chapter 17) suggested the sequential procedure for nonlinear model that starts with initial guess of the parameter values, then finds the optimum design for a linearized model sequentially until the new parameter estimate is sufficiently accurate. Fedorov and Wu (2007), and Dragalin, Fedorov, and Wu (2008) used two-stage designs for dose-finding studies with two correlated responses. Dette, Bornkamp and Bretz (2013) compared the asymptotic efficiency of two-stage adaptive optimal designs with fixed designs.

Let $\boldsymbol{\theta}_{0}$ be an initial guess of true parameter $\boldsymbol{\theta}$. In two-stage adaptive optimal designs, first take $n_{0}$ observations from the locally optimal design $\xi_{\boldsymbol{\theta}_{0}}$; then estimate $\boldsymbol{\theta}_{0}$ by the method of maximum likelihood. Let $\widehat{\boldsymbol{\theta}}_{0}$ be the estimate of $\boldsymbol{\theta}_{0}$ from the first stage, and let $\xi_{\widehat{\boldsymbol{\theta}}_{0}}$ be the locally optimal design based on $\widehat{\boldsymbol{\theta}}_{0}$. Take $n_{1}$ observations from the locally optimal design $\xi_{\widehat{\boldsymbol{\theta}}_{0}}$. Then $\widehat{\boldsymbol{\theta}}$ is the maximum likelihood estimate from all observations $n=n_{0}+n_{1}$. Since the second stage design is a function of the maximum likelihood estimates from the first stage (which are functions of the sufficient statistics from the first stage), the data from the second stage depends on the data from the first stage.

To aid in selecting the size of $n_{0}$, Pronzato and Pázman (2013, Section 8.5) noted that the asymptotically optimal allocation of $n_{0}$ is proportional to $\sqrt{n}$. Lane, Yao, and Flournoy (2013) showed that using $n_{0}=\sqrt{n}$ is not necessarily optimal, but we 
choose $n_{0}$ to be the closest integer to $\sqrt{n}$ for simplicity. We recommend that the interested readers see the procedure for choosing $n_{0}$ that is recommended by Lane, Yao, and Flournoy.

Now we consider two possible cases. In the first case, $\boldsymbol{\theta}_{0}$ is a very inefficient choice. Among the parameters in Table 4.3, $\boldsymbol{\theta}_{16}$ is the least efficient choice if the true parameter is $\boldsymbol{\theta}_{2}$. Its efficiency is $\left(\left|M\left(\xi_{\boldsymbol{\theta}_{16}}, \boldsymbol{\theta}_{2}\right)\right| /\left|M\left(\xi_{\boldsymbol{\theta}_{2}}, \boldsymbol{\theta}_{2}\right)\right|\right)^{1 / m}=.2707$. We assumed $\boldsymbol{\theta}_{2}$ is the true parameter, and used $\boldsymbol{\theta}_{16}$ as the initial parameter in stage 1 of a twostage design. The locally optimal designs and probability distributions for $\boldsymbol{\theta}_{2}$ and $\boldsymbol{\theta}_{16}$ are in Table 4.3 and Figure 4.10. Figure 4.11 (a) shows us the efficiency of two-stage adaptive optimal designs with various sample sizes. Simulation details are given in Appendix A.6. Compared to the efficiency of the initial locally optimal design (dashed line), two-stage adaptive optimal designs have substantially larger information for the true parameters. It clearly demonstrates that one can compensate for the initial misspecified parameters by using two-stage adaptive optimal designs.

In the second case, $\boldsymbol{\theta}_{0}$ is pretty efficient. We used $\boldsymbol{\theta}_{2}$ as the true parameter, and $\boldsymbol{\theta}_{10}$ as the initial parameter used in stage 1 of a two-stage design. Among the parameters in Table $4.3, \boldsymbol{\theta}_{10}$ is the most efficient choice if the true parameter is $\boldsymbol{\theta}_{2}$. The locally optimal designs and probability distributions for $\boldsymbol{\theta}_{10}$ may be found in Table 4.3 and Figure 4.10 , and the efficiency, $\left(\left|M\left(\xi_{\boldsymbol{\theta}_{10}}, \boldsymbol{\theta}_{2}\right)\right| /\left|M\left(\xi_{\boldsymbol{\theta}_{2}}, \boldsymbol{\theta}_{2}\right)\right|\right)^{1 / m}$, is .8436. Figure 4.11 (b) shows us that although there is less room for gains in efficiency, two-stage adaptive optimal designs still are extremely likely to have larger information than the initial locally optimal design. In summary, a two-stage adaptive optimal design is highly recommended over a single stage design. 


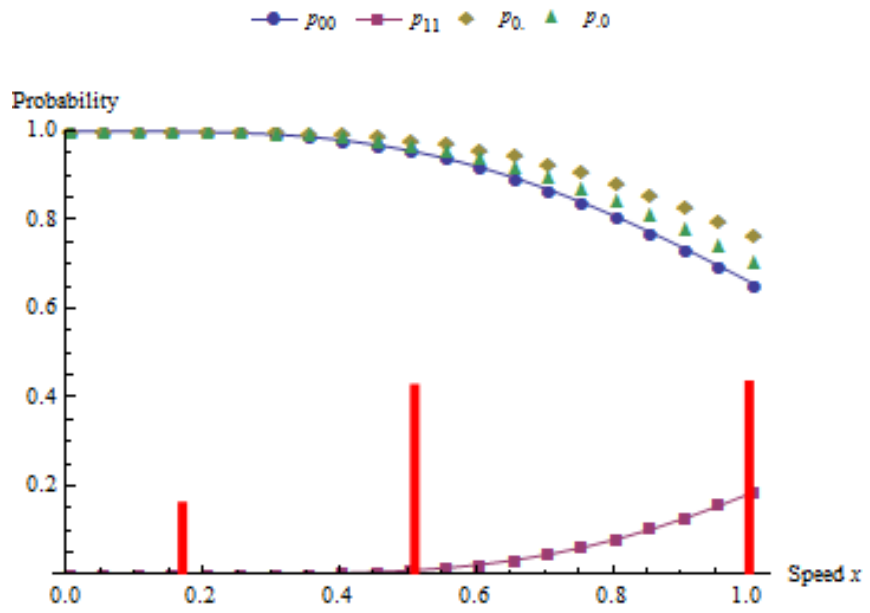

(a) $\boldsymbol{\theta}_{2}=\{-3,4,5,2,2, .1, .2\}$

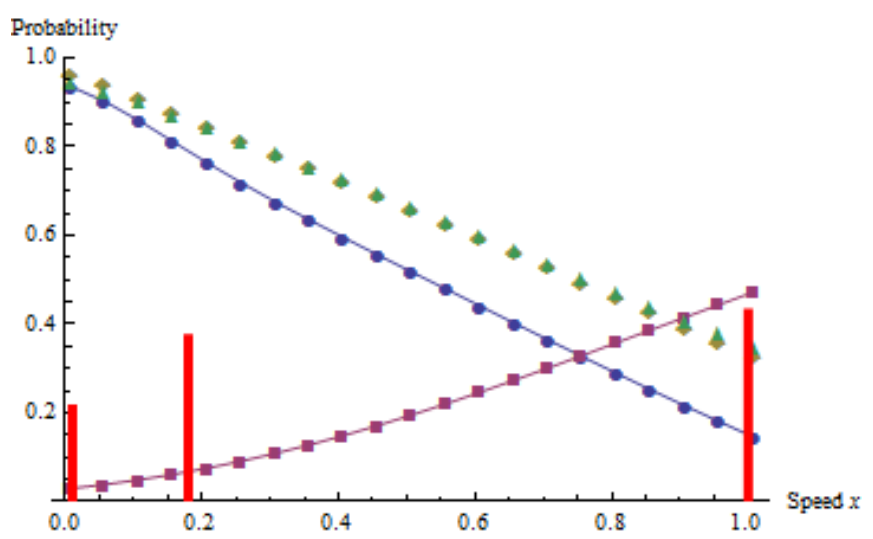

(b) $\boldsymbol{\theta}_{16}=\{-1.5,8,10,1.5,1, .3, .4\}$

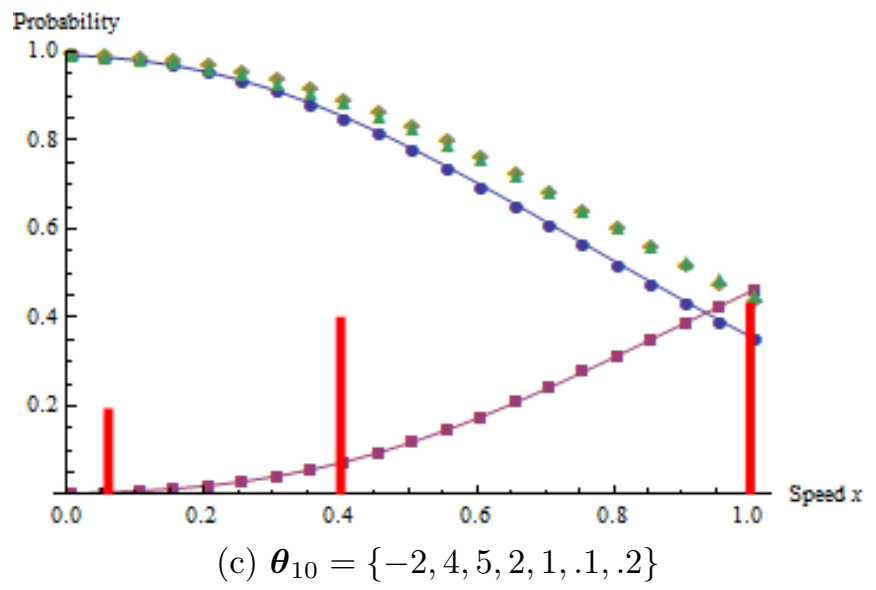

Figure 4.10: Probability distributions and D-optimal designs with $\boldsymbol{\theta}_{2}, \boldsymbol{\theta}_{16}$, and $\boldsymbol{\theta}_{10}$ 


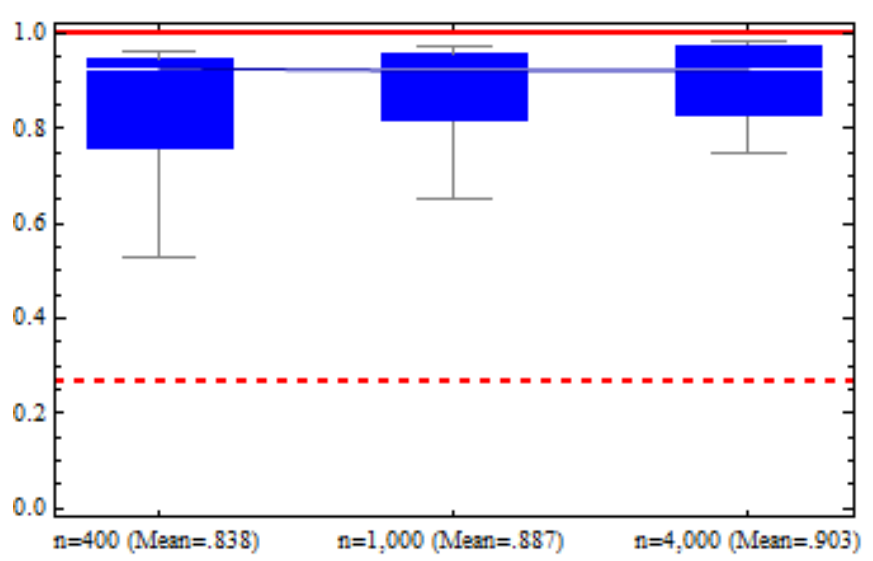

(a) Case 1

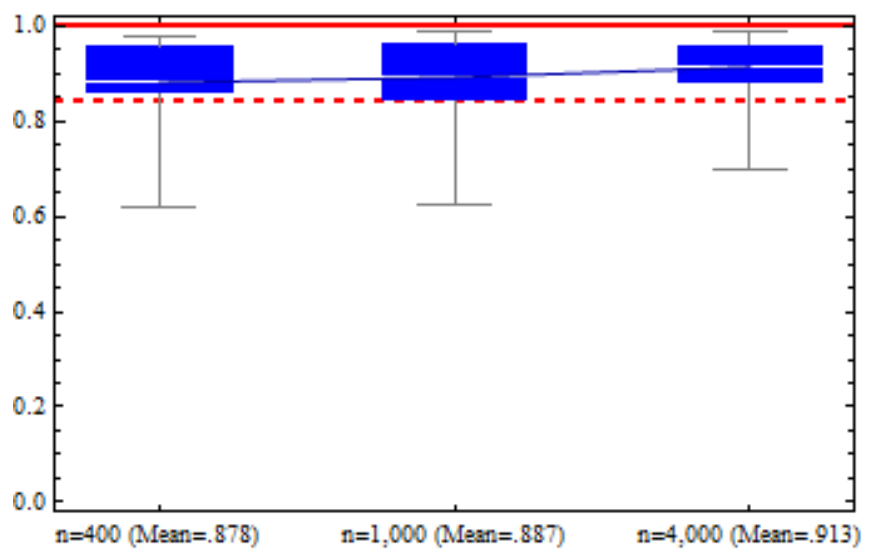

(b) Case 2

Figure 4.11: Boxplots of the efficiency of the two-stage adaptive optimal design relative to the initial locally optimal design $\xi_{\boldsymbol{\theta}_{0}}$. The efficiency of the locally optimal design $\xi_{\boldsymbol{\theta}_{0}}$ (used in the first stage of the adaptive procedure) is shown by the dashed line. $\xi_{\widehat{\boldsymbol{\theta}}_{0}}$ denotes a locally optimal design based on parameter estimates from the first stage design of $\xi_{\boldsymbol{\theta}_{0}}$.

$$
\begin{gathered}
\text { Efficiency }=\left(\frac{p_{0}\left|M\left(\xi_{\boldsymbol{\theta}_{0}}, \boldsymbol{\theta}\right)\right|+\left(1-p_{0}\right)\left|M\left(\xi_{\widehat{\boldsymbol{\theta}}_{0}}, \boldsymbol{\theta}\right)\right|}{\left|M\left(\xi_{\boldsymbol{\theta}}, \boldsymbol{\theta}\right)\right|}\right)^{1 / m} \text { where } p_{0}=\frac{\sqrt{n}}{n}, \\
\text { Dashed line: }\left(\frac{\mid M\left(\xi_{\boldsymbol{\theta}_{0}}, \boldsymbol{\theta} \mid\right.}{\left|M\left(\xi_{\boldsymbol{\theta}}, \boldsymbol{\theta}\right)\right|}\right)^{1 / m} \cdot \\
* * 100 \text { experiments have been performed for each } n .
\end{gathered}
$$




\section{Chapter 5}

\section{A Trivariate Serial System subjected to Decreasing Damage}

\subsection{A Trivariate System with Components having Decreasing Damage}

\subsubsection{Motivation}

Consider a serial system such as is depicted in Figure 5.1. Notice that the system has three components, and a physical stress (radiation) comes from a point source. The ellipses in Figure 5.1 (b) mark the components. In Figure 5.1 (c), distances are marked at which radiation measurements are taken. So the amount of damage on the first component $\left(Y_{1}\right)$ is always greater than the second component $\left(Y_{2}\right)$, and the amount of damage on the second component $\left(Y_{2}\right)$ is always greater than the third one 
$\left(Y_{3}\right)$ from the outside stress (radiation).

Each component is considered to have "failed" if the amount of damage it receives is greater than some predetermined cutoff value. In our example, it might be detectable radiation, or radiation levels above which are considered harmful. We define component failure indicators by

$$
\begin{aligned}
& U_{1}= \begin{cases}0 \text { (No failure for the component } 1), & \text { if } Y_{1}<v_{1}^{*} \\
1 \text { (Failure for the component }), & \text { otherwise }\end{cases} \\
& U_{2}= \begin{cases}0 \text { (No failure for the component } 2), & \text { if } Y_{2}<v_{2}^{*} \\
1 \text { (Failure for the component } 2), & \text { otherwise, }\end{cases} \\
& U_{3}= \begin{cases}0 \text { (No failure for the component } 3), & \text { if } Y_{3}<v_{3}^{*} \\
1 \text { (Failure for the component } 3), & \text { otherwise, }\end{cases}
\end{aligned}
$$

where $v_{1}^{*}, v_{2}^{*}$ and $v_{3}^{*}$ are the predetermined cutoff values. For simplicity, we assume identical predetermined cutoff values $v^{*}\left(=v_{1}^{*}=v_{2}^{*}=v_{3}^{*}\right)$. So there exist eight possible outcome probabilities given a stress $x$ :

$$
p_{u_{1} u_{2} u_{3}}(x)=\operatorname{Pr}\left(U_{1}=u_{1}, U_{2}=u_{2}, U_{3}=u_{3} \mid X=x\right), \quad\left\{u_{1}, u_{2}, u_{3}\right\} \in\{0,1\} .
$$

The eight possible outcomes and their probabilities are defined in Table 5.1. Since we assume a system with deceasing amount of damage $\left(Y_{1}>Y_{2}>Y_{3}\right), p_{001}=p_{010}=$ $p_{011}=p_{101}=0$. In words, a component failure can't occur before the previous component fails. 


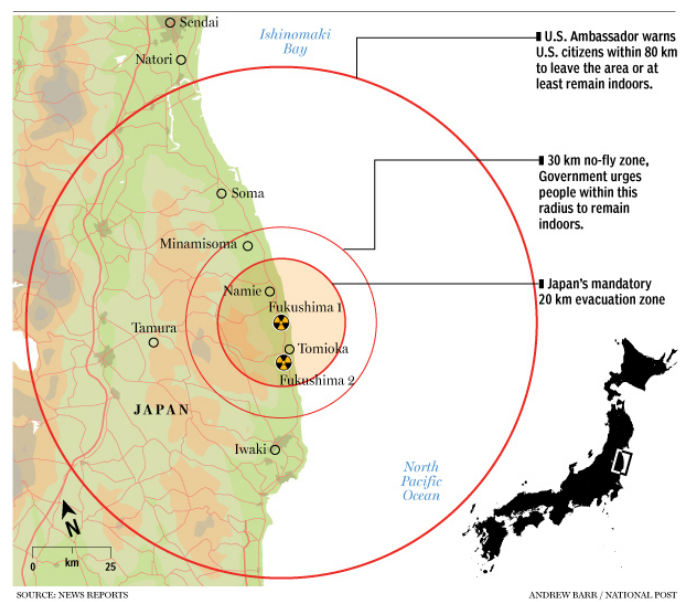

(a) Evacuation three zones near Fukushima (Ref: News reports)

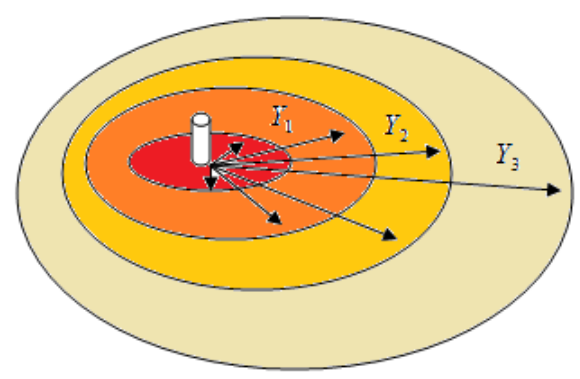

(b) Decreasing amount of damage from a radiation source point

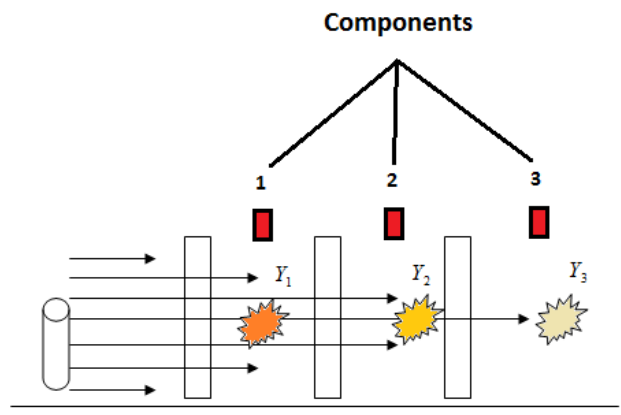

(c) Distances at which radiation measurements are taken

Figure 5.1: System with decreasing amount of damage 
No Failure on Component 1

Component 2

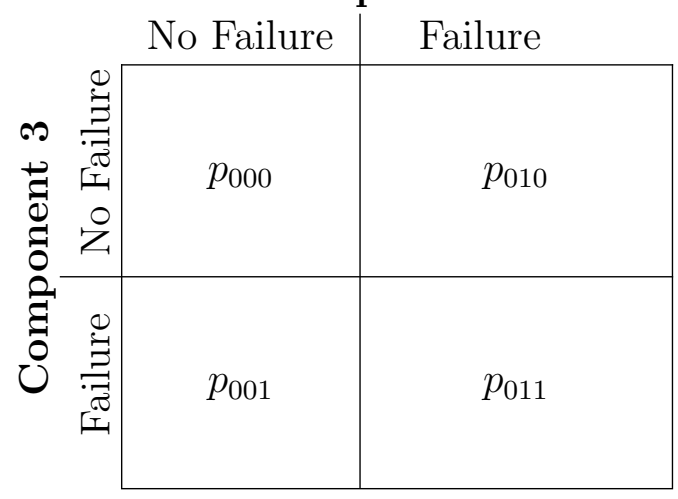

Failure on Component 1 Component 2

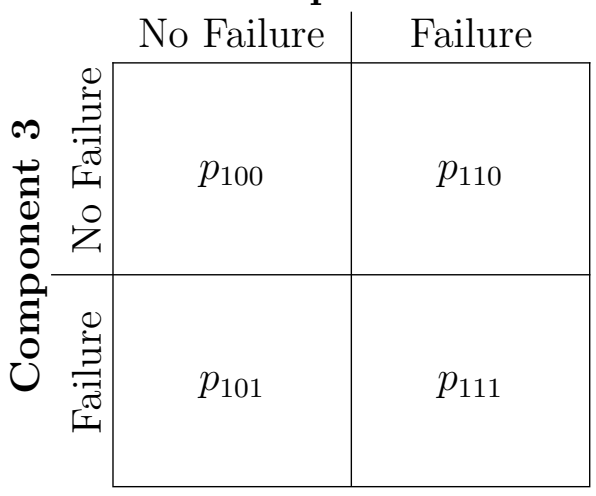

Table 5.1: Outcomes and their probabilities

The system is said to fail when all components fail. In mechanical engineering, it is important to estimate the amount of physical stress having a predetermined probability of system failure where developing new machine products. To estimate this stress most efficiently in terms of time and cost, a locally optimal experimental design is considered. The stress that corresponds to a given failure probability is called the target stress. With predictor functions, a target stress can be written as a function of parameters. To estimate the targeted stress, which here is called an effective stress, with minimum variance efficiently, it is important to find a c-optimal experimental design. The c-optimality criterion reflects to an interest in estimating a function of the parameters with minimum variance. 


\subsubsection{A Trivariate Weibull Model}

Begin with the bivariate exponential density that was introduced by Freund (1961):

$$
f\left(t_{1}, t_{2}\right)= \begin{cases}\beta_{1} \beta_{2 \mid 1} e^{-\beta_{2 \mid 1} t_{2}-\left(\beta_{1}+\beta_{2}-\beta_{2 \mid 1}\right) t_{1}} & \text { for } 0<t_{1}<t_{2}<\infty \\ \beta_{2} \beta_{1 \mid 2} e^{-\beta_{1 \mid 2} t_{1}-\left(\beta_{1}+\beta_{2}-\beta_{1 \mid 2}\right) t_{2}} & \text { for } 0<t_{2}<t_{1}<\infty\end{cases}
$$

The marginal density functions of (5.2) can be written as weighted univariate exponential density functions:

$$
\begin{aligned}
& f\left(t_{1}\right)=\left(a_{1}\right) \beta_{1 \mid 2} e^{-\beta_{1 \mid 2} t_{1}}+\left(1-a_{1}\right)\left(\beta_{1}+\beta_{2}\right) e^{-\left(\beta_{1}+\beta_{2}\right) t_{1}} \\
& f\left(t_{2}\right)=\left(a_{2}\right) \beta_{2 \mid 1} e^{-\beta_{2 \mid 1} t_{2}}+\left(1-a_{2}\right)\left(\beta_{1}+\beta_{2}\right) e^{-\left(\beta_{1}+\beta_{2}\right) t_{2}}
\end{aligned}
$$

where $a_{1}=\left(\frac{\beta_{2}}{\beta_{1}+\beta_{2}-\beta_{1 \mid 2}}\right), a_{2}=\left(\frac{\beta_{1}}{\beta_{1}+\beta_{2}-\beta_{2 \mid 1}}\right)$. If $\beta_{1}=\beta_{1 \mid 2}$, and $\beta_{2}=\beta_{2 \mid 1}$, then $a_{1}=$ $a_{2}=1$ and (5.3) reduces to two independent univariate exponential density functions.

A trivariate exponential probability density can be formed as an extension of Freund's bivariate exponential density. Figure 5.2 shows the parameter structure given $t_{1}<t_{2}<t_{3}$ and the density is 


$$
\begin{aligned}
& f\left(t_{1}, t_{2}, t_{3}\right) \\
& = \begin{cases}\beta_{1} \beta_{2 \mid 1} \beta_{3 \mid 21} e^{-\left(\beta_{1}+\beta_{2}+\beta_{3}-\beta_{2 \mid 1}-\beta_{3 \mid 1}\right) t_{1}-\left(\beta_{2 \mid 1}+\beta_{3 \mid 1}-\beta_{3 \mid 21}\right) t_{2}-\beta_{3 \mid 21} t_{3}} & \text { for } 0<t_{1}<t_{2}<t_{3}<\infty, \\
\beta_{1} \beta_{3 \mid 1} \beta_{2 \mid 31} e^{-\left(\beta_{1}+\beta_{2}+\beta_{3}-\beta_{3 \mid 1}-\beta_{2 \mid 1}\right) t_{1}-\left(\beta_{3 \mid 1}+\beta_{2 \mid 1}-\beta_{2 \mid 31}\right) t_{3}-\beta_{2 \mid 31} t_{2}} & \text { for } 0<t_{1}<t_{3}<t_{2}<\infty, \\
\beta_{2} \beta_{1 \mid 2} \beta_{3 \mid 12} e^{-\left(\beta_{1}+\beta_{2}+\beta_{3}-\beta_{1 \mid 2}-\beta_{3 \mid 2}\right) t_{2}-\left(\beta_{1 \mid 2}+\beta_{3 \mid 2}-\beta_{3 \mid 12}\right) t_{1}-\beta_{3 \mid 12} t_{3}} & \text { for } 0<t_{2}<t_{1}<t_{3}<\infty, \\
\beta_{2} \beta_{3 \mid 2} \beta_{1 \mid 32} e^{-\left(\beta_{1}+\beta_{2}+\beta_{3}-\beta_{3 \mid 2}-\beta_{1 \mid 2}\right) t_{2}-\left(\beta_{3 \mid 2}+\beta_{1 \mid 2}-\beta_{1 \mid 32}\right) t_{3}-\beta_{1 \mid 32} t_{1}} & \text { for } 0<t_{2}<t_{3}<t_{1}<\infty, \\
\beta_{3} \beta_{1 \mid 3} \beta_{2 \mid 13} e^{-\left(\beta_{1}+\beta_{2}+\beta_{3}-\beta_{1 \mid 2}-\beta_{2 \mid 3}\right) t_{3}-\left(\beta_{1 \mid 3}+\beta_{2 \mid 3}-\beta_{2 \mid 13}\right) t_{1}-\beta_{2 \mid 13} t_{2}} & \text { for } 0<t_{3}<t_{1}<t_{2}<\infty, \\
\beta_{3} \beta_{2 \mid 3} \beta_{1 \mid 23} e^{-\left(\beta_{1}+\beta_{2}+\beta_{3}-\beta_{2 \mid 3}-\beta_{1 \mid 3}\right) t_{3}-\left(\beta_{2 \mid 3}+\beta_{1 \mid 3}-\beta_{1 \mid 23}\right) t_{2}-\beta_{1 \mid 23} t_{1}} & \text { for } 0<t_{3}<t_{2}<t_{1}<\infty\end{cases}
\end{aligned}
$$

We create a trivariate Weibull probability density from (5.4) using power transformations $T_{1}=Y_{1}^{\tau}, T_{2}=Y_{2}^{\tau}$, and $T_{3}=Y_{3}^{\tau}$ with $\tau>0$, and simplify to obtain

$$
\begin{aligned}
& f\left(y_{1}, y_{2}, y_{3}\right) \\
& =\beta_{3} \beta_{2|3|} \beta_{1 \mid 23} \tau^{3}\left(y_{1} y_{2} y_{3}\right)^{\tau-1} e^{-\left(\beta_{1}+\beta_{2}+\beta_{3}-\beta_{2 \mid 3}-\beta_{1 \mid 3}\right) y_{3}^{\tau}-\left(\beta_{2 \mid 3}+\beta_{1 \mid 3}-\beta_{1 \mid 23}\right) y_{2}^{\tau}-\beta_{1 \mid 23} y_{1}^{\tau}} \\
& \text { for } 0<y_{3}<y_{2}<y_{1}<\infty .
\end{aligned}
$$

The density for other values of $y_{1}, y_{2}$ and $y_{3}$ is given in (A.10) of Appendix A.8. One can see that (A.10) reduces to the trivariate exponential density (5.4) if $\tau=1$.

To make $p_{001}, p_{010}, p_{011}$, and $p_{101}$ zero with respect to any $v^{*}$, and satisfy the condition $Y_{1}>Y_{2}>Y_{3}$, we set $\beta_{1}, \beta_{2}, \beta_{2 \mid 1}, \beta_{3 \mid 1}, \beta_{1 \mid 3}, \beta_{1 \mid 2}, \beta_{3 \mid 2}, \beta_{3 \mid 21}, \beta_{2 \mid 31}, \beta_{3 \mid 12}, \beta_{1 \mid 32}$, $\beta_{2 \mid 13}$ to zero, and the equation (A.10) becomes

$$
g\left(y_{1}, y_{2}, y_{3}\right)=\beta_{3} \beta_{2 \mid 3} \beta_{1 \mid 23} \tau^{3}\left(y_{1} y_{2} y_{3}\right)^{\tau-1} e^{-\left(\beta_{3}-\beta_{2 \mid 3}\right) y_{3}^{\tau}-\left(\beta_{2 \mid 3}-\beta_{1 \mid 23}\right) y_{2}^{\tau}-\beta_{1 \mid 23} y_{1}^{\tau}}
$$


for $0<y_{3}<y_{2}<y_{1}<\infty . g\left(y_{1}, y_{2}, y_{3}\right)$ is also a probability density function.

The marginal density functions of the trivariate Weibull density (5.6) are

$$
\begin{aligned}
& g\left(y_{1}\right)=\left(\gamma_{11}\right) \beta_{1 \mid 23} \tau y_{1}^{\tau-1} e^{-\beta_{1 \mid 23} y_{1}^{\tau}}+\left(\gamma_{12}\right) \beta_{2 \mid 3} \tau y_{1}^{\tau-1} e^{-\beta_{2 \mid 3} y_{1}^{\tau}}+\left(1-\gamma_{11}-\gamma_{12}\right) \beta_{3} \tau y_{1}^{\tau-1} e^{-\beta_{3} y_{1}^{\tau}} \\
& g\left(y_{2}\right)=\left(\gamma_{2}\right) \beta_{2 \mid 3} \tau y_{2}^{\tau-1} e^{-\beta_{2 \mid 3} y_{2}^{\tau}}+\left(1-\gamma_{2}\right) \beta_{3} \tau y_{2}^{\tau-1} e^{-\beta_{3} y_{2}^{\tau}} \\
& g\left(y_{3}\right)=\beta_{3} \tau y_{3}^{\tau-1} e^{-\beta_{3} y_{3}^{\tau}}
\end{aligned}
$$

where

$$
\begin{aligned}
\gamma_{11} & =\frac{\beta_{3} \beta_{2 \mid 3}\left(\beta_{3}-\beta_{2 \mid 3}\right)}{\left(\beta_{3}-\beta_{2 \mid 3}\right)\left(\beta_{2 \mid 3}-\beta_{1 \mid 23}\right)\left(\beta_{3}-\beta_{1 \mid 23}\right)}, \\
\gamma_{12} & =-\frac{\beta_{3} \beta_{1 \mid 23}\left(\beta_{3}-\beta_{1 \mid 23}\right)}{\left(\beta_{3}-\beta_{2 \mid 3}\right)\left(\beta_{2 \mid 3}-\beta_{1 \mid 23}\right)\left(\beta_{3}-\beta_{1 \mid 23}\right)}, \\
\gamma_{2} & =\frac{\beta_{3}}{\beta_{3}-\beta_{2 \mid 3}} .
\end{aligned}
$$

In $(5.6), p_{001}, p_{010}, p_{011}$, and $p_{101}$ are exactly zero at any $v^{*}$, and other outcome probabilities can be computed:

$$
\begin{aligned}
& p_{000}=\int_{0}^{v^{*}} \int_{0}^{y_{1}} \int_{0}^{y_{2}} g\left(y_{1}, y_{2}, y_{3}\right) d y_{3} d y_{2} d y_{1} ; \\
& p_{100}=\int_{v^{*}}^{\infty} \int_{0}^{v^{*}} \int_{0}^{y_{2}} g\left(y_{1}, y_{2}, y_{3}\right) d y_{3} d y_{2} d y_{1} ; \\
& p_{110}=\int_{v^{*}}^{\infty} \int_{v^{*}}^{y_{1}} \int_{0}^{v^{*}} g\left(y_{1}, y_{2}, y_{3}\right) d y_{3} d y_{2} d y_{1} ; \\
& p_{111}=\int_{v^{*}}^{\infty} \int_{v^{*}}^{y_{1}} \int_{v^{*}}^{y_{2}} g\left(y_{1}, y_{2}, y_{3}\right) d y_{3} d y_{2} d y_{1} .
\end{aligned}
$$

These outcome probabilities are evaluated in Appendix A.9. 


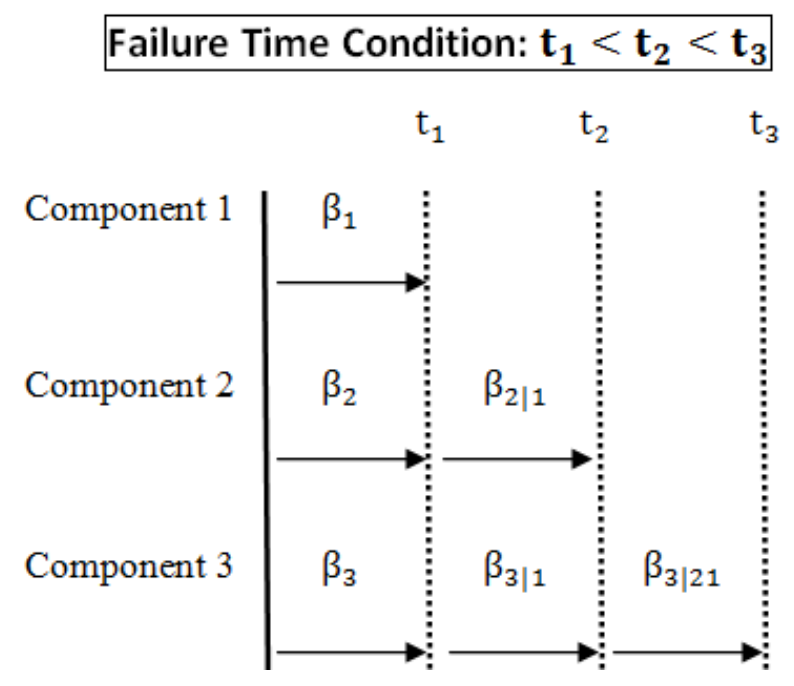

Figure 5.2: Failure rate changes after component failures at $t_{1}, t_{2}$, and $t_{3}$ given $t_{1}<t_{2}<t_{3}$ under a trivariate exponential model. 


\subsubsection{A Trivariate Weibull Regression Model}

Chapter 4.1.2 proposed a bivariate Weibull regression model. Analogously, regression terms can be inserted into (5.6):

$$
\begin{aligned}
& -\log \left(\beta_{3}\right)=\eta_{1}\left(\boldsymbol{x} ; \boldsymbol{\vartheta}_{1}\right) \\
& -\log \left(\beta_{2 \mid 3}\right)=\eta_{2}\left(\boldsymbol{x} ; \boldsymbol{\vartheta}_{2}\right) \\
& -\log \left(\beta_{1 \mid 23}\right)=\eta_{3}\left(\boldsymbol{x} ; \boldsymbol{\vartheta}_{3}\right)
\end{aligned}
$$

where $\eta_{1}=\eta_{1}\left(\boldsymbol{x} ; \boldsymbol{\vartheta}_{1}\right), \eta_{2}=\eta_{2}\left(\boldsymbol{x} ; \boldsymbol{\vartheta}_{2}\right)$, and $\eta_{3}=\eta_{3}\left(\boldsymbol{x} ; \boldsymbol{\vartheta}_{3}\right)$ may be nonlinear functions of covariates $\boldsymbol{x}$ and regression parameters $\boldsymbol{\vartheta}_{1}, \boldsymbol{\vartheta}_{2}$ and $\boldsymbol{\vartheta}_{3}$. Expressions for the outcome probabilities in terms of the regression parameters are given in Appendix A.10.

\subsection{Information Matrix and a Locally Optimal De- sign}

Inserting (5.8) into (5.7), the outcome probabilities are functions of $\boldsymbol{\theta}=\left\{\boldsymbol{\vartheta}_{1}, \boldsymbol{\vartheta}_{2}, \boldsymbol{\vartheta}_{3}, \tau\right\}$, the set of model parameters. We omit the dependence of the outcome probabilities on $\boldsymbol{\theta}$ for notational brevity. The log-likelihood function is

$$
\begin{aligned}
& l\left(\boldsymbol{\theta} \mid u_{1}, u_{2}, u_{3} ; \boldsymbol{x}\right) \\
& =\left(u_{1} u_{2} u_{3}\right) \ln \left(p_{111}\right)+\left(u_{1} u_{2}\left(1-u_{3}\right)\right) \ln \left(p_{110}\right)+\left(u_{1}\left(1-u_{2}\right)\left(1-u_{3}\right)\right) \ln \left(p_{100}\right) \\
& +\left(\left(1-u_{1}\right)\left(1-u_{2}\right)\left(1-u_{3}\right)\right) \ln \left(1-p_{111}-p_{110}-p_{100}\right),
\end{aligned}
$$


and the score function is $\partial l\left(\boldsymbol{\theta} \mid u_{1}, u_{2}, u_{3} ; \boldsymbol{x}\right) / \partial \boldsymbol{\theta}=A B C$, where

$$
\begin{gathered}
A=\left[\begin{array}{llll}
\frac{\partial \eta_{1}\left(\boldsymbol{x} ; \boldsymbol{\vartheta}_{1}\right)}{\partial \boldsymbol{\vartheta}_{1}} & \frac{\partial \eta_{2}\left(\boldsymbol{x} ; \boldsymbol{\vartheta}_{2}\right)}{\partial \boldsymbol{\vartheta}_{2}} & \frac{\partial \eta_{3}\left(\boldsymbol{x} ; \boldsymbol{\vartheta}_{3}\right)}{\partial \boldsymbol{\vartheta}_{3}} & 1
\end{array}\right] \times \mathbf{1}_{3 \times 1}, \\
\frac{\partial \eta_{j}\left(\boldsymbol{x} ; \boldsymbol{\vartheta}_{j}\right)}{\partial \boldsymbol{\vartheta}_{j}}=\left[\begin{array}{llll}
\frac{\partial \eta_{j}\left(\boldsymbol{x} ; \boldsymbol{\vartheta}_{j}\right)}{\partial \vartheta_{j 1}} & \frac{\partial \eta_{j}\left(\boldsymbol{x} ; \boldsymbol{\vartheta}_{j}\right)}{\partial \vartheta_{j 2}} & \ldots & \frac{\partial \eta_{j}\left(\boldsymbol{x} ; \boldsymbol{\vartheta}_{j}\right)}{\partial \vartheta_{j m_{j}}}
\end{array}\right], j=1,2,3,
\end{gathered}
$$

$m_{j}$ is the number of parameters in $\boldsymbol{\vartheta}_{j}$ and $\mathbf{1}_{a \times b}$ is a $a \times b$ matrix of ones;

$$
\begin{aligned}
& B=\left[\begin{array}{ccc}
\frac{\partial p_{111}}{\partial \eta_{1}\left(\boldsymbol{x} ; \boldsymbol{\vartheta}_{1}\right)} \times \mathbf{1}_{m_{1} \times 1} & \frac{\partial p_{110}}{\partial \eta_{1}\left(\boldsymbol{x} ; \boldsymbol{\vartheta}_{1}\right)} \times \mathbf{1}_{m_{1} \times 1} & \frac{\partial p_{100}}{\partial \eta_{1}\left(\boldsymbol{x} ; \boldsymbol{\vartheta}_{1}\right)} \times \mathbf{1}_{m_{1} \times 1} \\
\frac{\partial p_{111}}{\partial \eta_{2}\left(\boldsymbol{x} ; \boldsymbol{\vartheta}_{2}\right)} \times \mathbf{1}_{m_{2} \times 1} & \frac{\partial p_{110}}{\partial \eta_{2}\left(\boldsymbol{x} ; \boldsymbol{\vartheta}_{2}\right)} \times \mathbf{1}_{m_{2} \times 1} & \frac{\partial p_{100}}{\partial \eta_{1}\left(\boldsymbol{x} ; \boldsymbol{\vartheta}_{1}\right)} \times \mathbf{1}_{m_{2} \times 1} \\
\frac{\partial p_{111}}{\partial \eta_{3}\left(\boldsymbol{x} ; \boldsymbol{\vartheta}_{3}\right)} \times \mathbf{1}_{m_{3} \times 1} & \frac{\partial p_{110}}{\partial \eta_{3}\left(\boldsymbol{x} ; \boldsymbol{\vartheta}_{3}\right)} \times \mathbf{1}_{m_{3} \times 1} & \frac{\partial p_{100}}{\partial \eta_{1}\left(\boldsymbol{x} ; \boldsymbol{\vartheta}_{1}\right)} \times \mathbf{1}_{m_{3} \times 1} \\
\frac{\partial p_{111}}{\partial \tau} & \frac{\partial p_{110}}{\partial \tau} & \frac{\partial p_{100}}{\partial \tau}
\end{array}\right] \\
& C=\left[\begin{array}{c}
\frac{u_{1} u_{2} u_{3}}{p_{111}}-\frac{\left(1-u_{1}\right)\left(1-u_{2}\right)\left(1-u_{3}\right)}{1-p_{111}-p_{110}-p_{100}} \\
\frac{u_{1} u_{2}\left(1-u_{3}\right)}{p_{110}}-\frac{\left(1-u_{1}\right)\left(1-u_{2}\right)\left(1-u_{3}\right)}{1-p_{111}-p_{110}-p_{100}} \\
\frac{u_{1}\left(1-u_{2}\right)\left(1-u_{3}\right)}{p_{100}}-\frac{\left(1-u_{1}\right)\left(1-u_{2}\right)\left(1-u_{3}\right)}{1-p_{111}-p_{110}-p_{100}}
\end{array}\right] .
\end{aligned}
$$


The information matrix for a single system stress is

$$
\begin{aligned}
\mu(x, \boldsymbol{\theta}) & =\mathrm{E}\left[\left(\frac{\partial l(\boldsymbol{\theta} \mid u, v ; \boldsymbol{x})}{\partial \boldsymbol{\theta}}\right)\left(\frac{\partial l(\boldsymbol{\theta} \mid u, v ; \boldsymbol{x})}{\partial \boldsymbol{\theta}}\right)^{T}\right]=\mathrm{E}\left[(A B C)(A B C)^{T}\right] \\
& =\mathrm{E}\left[A B C C^{T} B^{T} A^{T}\right]=A B\left[\mathrm{E}\left(C C^{T}\right)\right] B^{T} A^{T} .
\end{aligned}
$$

Since $U_{1}, U_{2}$ and $U_{3}$ are dependent, $\mathrm{E}\left(C C^{T}\right)$ is

$$
\begin{aligned}
& {\left[\begin{array}{ccc}
\frac{1}{p_{111}}+\frac{1}{1-p_{111}-p_{110}-p_{100}} & \frac{1}{1-p_{111}-p_{110}-p_{100}} & \frac{1}{1-p_{111}-p_{110}-p_{100}} \\
\frac{1}{1-p_{111}-p_{110}-p_{100}} & \frac{1}{p_{110}}+\frac{1}{1-p_{111}-p_{110}-p_{100}} & \frac{1}{1-p_{111}-p_{110}-p_{100}} \\
\frac{1}{1-p_{111}-p_{110}-p_{100}} & \frac{1}{1-p_{111}-p_{110}-p_{100}} & \frac{1}{p_{100}}+\frac{1}{1-p_{111}-p_{110}-p_{100}}
\end{array}\right]} \\
& =\operatorname{diag}\left(\frac{1}{p_{111}}, \frac{1}{p_{110}}, \frac{1}{p_{100}}\right)+\left(\frac{1}{1-p_{111}-p_{110}-p_{100}}\right) \mathbf{1}_{3 \times 3} .
\end{aligned}
$$

For linear predictor functions, equation (5.8) becomes

$$
\begin{aligned}
& -\log \left(\beta_{3}\right)=\theta_{0}+\theta_{1} x ; \\
& -\log \left(\beta_{2 \mid 3}\right)=\theta_{0}+\theta_{2} x ; \\
& -\log \left(\beta_{1 \mid 23}\right)=\theta_{0}+\theta_{3} x
\end{aligned}
$$

and the product of (5.10) and (5.11) becomes

$$
G^{T}=(A B)^{T}=\left[\begin{array}{lllll}
\frac{\partial p_{111}}{\partial \theta_{0}} & \frac{\partial p_{111}}{\partial \theta_{1}} & \frac{\partial p_{111}}{\partial \theta_{2}} & \frac{\partial p_{111}}{\partial \theta_{3}} & \frac{\partial p_{111}}{\partial \tau} \\
\frac{\partial p_{110}}{\partial \theta_{0}} & \frac{\partial p_{110}}{\partial \theta_{1}} & \frac{\partial p_{110}}{\partial \theta_{2}} & \frac{\partial p_{110}}{\partial \theta_{3}} & \frac{\partial p_{110}}{\partial \tau} \\
\frac{\partial p_{100}}{\partial \theta_{0}} & \frac{\partial p_{100}}{\partial \theta_{1}} & \frac{\partial p_{100}}{\partial \theta_{2}} & \frac{\partial p_{100}}{\partial \theta_{3}} & \frac{\partial p_{100}}{\partial \tau}
\end{array}\right]
$$

Note that the first and the last columns of the matrix $G^{T}$ are linearly dependent 
assuming the probability density function (5.6), we remove the last column of the matrix $G^{T}$. The average information matrix for an experiment using design $\xi$ is

$$
M(\xi, \boldsymbol{\theta})=\sum_{i=1}^{K} w_{i} \mu\left(x_{i}, \boldsymbol{\theta}\right) .
$$

Then a locally optimal design $\xi^{*}$ is defined as

$$
\xi^{*}=\arg \min _{\xi \in \Xi(\mathfrak{X})} \Psi(M(\xi, \boldsymbol{\theta})),
$$

where $\Xi(\mathfrak{X})$ is a set of all possible combinations of design weights and design points in a sample space $\mathfrak{X}$.

\section{3 c-Optimal Designs for an Effective Stress}

Let $E S_{\alpha}$ denote an "effective stress", which is defined explicitly below. Then the criterion we minimize is $\Psi(M(\xi, \boldsymbol{\theta}))=\operatorname{Var}\left[\widehat{E S}_{\alpha}\right]$. Define the outcome probability $p_{111}=P$ (Failure for all components $)$ as the probability of "system failure". Practitioners may want to estimate the level of stress, $\boldsymbol{x}$, at the predetermined probability of $\alpha=p_{111}$ with minimum variance. Alternatively, $\left(1-p_{111}\right)=P($ At least one component is working) may be considered. This section considers the effective stress at the probability of failure for all components:

$$
\alpha=\mathrm{P}(\text { Failure for all components })=p_{111}=\exp \left(-\beta_{3} v^{* \tau}\right),
$$


where $\alpha$ is a predetermined probability value. Inserting the linear predictor terms (5.12) into (5.15) yields

$$
\alpha=\mathrm{P}(\text { Failure for all components } \mid \text { stress } \boldsymbol{x})=p_{111}=\exp \left(-\left(e^{-\left(\theta_{0}+\theta_{1} x\right)}\right) v^{* \tau}\right) \text {. }
$$

Then the solution of (5.16) is an effective stress, $E S_{\alpha}$ :

$$
E S_{\alpha}=\frac{\tau \ln v^{*}-\ln \{\ln (1 / \alpha)\}-\theta_{0}}{\theta_{1}}
$$

To estimate $E S_{\alpha}$ most effectively, c-optimal designs are proposed. In this case, the c-optimality criterion is to minimize

$$
\Psi(M(\xi, \boldsymbol{\theta}))=\operatorname{Var}\left[\widehat{E S}_{\alpha}\right] \approx\left(E S_{\alpha}^{\prime}\right)^{T} M^{-1}(\xi, \boldsymbol{\theta}) E S_{\alpha}^{\prime}
$$

where

$$
\left(E S_{\alpha}^{\prime}\right)^{T}=\left[\frac{\partial E S_{\alpha}}{\partial \theta_{0}}, \frac{\partial E S_{\alpha}}{\partial \theta_{1}}, 0,0, \frac{\partial E S_{\alpha}}{\partial \tau}\right]
$$

Since $\partial E S_{\alpha} / \partial \theta_{0}$ is a linear function of $\partial E S_{\alpha} / \partial \tau$ assuming the probability density function (5.6), we remove $\partial E S_{\alpha} / \partial \tau$ from (5.17).

To begin a search for a c-optimal design, set plausible initial parameters. For example, $p_{000}$ should be 1 at $x=0$, and then decrease as $x$ increases; $p_{111}$ should increase as $x$ increases. We set $\boldsymbol{\theta}=\left\{\theta_{0}, \theta_{1}, \theta_{2}, \theta_{3}, \tau\right\}=\{-4.0,3.5,2.5,1.5,2\}$ as the initial parameters. The General Equivalence Theorem (Kiefer, J., Wolfowitz, J.(1960)) states that, for $\xi^{*}$ to be a c-optimal design,

$$
\operatorname{tr}\left\{\mu(x) M^{-1}\left(\xi^{*}\right) c c^{T} M^{-1}\left(\xi^{*}\right)\right\} \leq c^{T} M^{-1}\left(\xi^{*}\right) c,
$$


for all sample points $x \in \mathfrak{X}$; $c$ is a vector of partial derivatives of a function of parameters with respect to $\boldsymbol{\theta}$, and $\operatorname{tr}|\cdot|$ denotes the trace of a matrix. A proof of (5.18) is given in Appendix A.12. To estimate $E S_{\alpha}$ with minimum variance, a c-optimal design must satisfy the following condition

$$
\operatorname{tr}\left\{\mu(x) M^{-1}\left(\xi^{*}\right) E S_{\alpha}^{\prime}\left(E S_{\alpha}^{\prime}\right)^{T} M^{-1}\left(\xi^{*}\right)\right\} \leq\left(E S_{\alpha}^{\prime}\right)^{T} M^{-1}\left(\xi^{*}\right) E S_{\alpha}^{\prime} \approx \operatorname{Var}\left[\widehat{E S}_{\alpha}\right]
$$

for all sample points $x \in \mathfrak{X}$. To find a c-optimal design, this paper applied the exchange algorithm (cf. Fedorov, and Hackl (1997), Chapter 3). More details are in Appendix A.13.

The bars in Figure 5.3 (a) represent the proportion of subjects allocated to different stresses for the c-optimal design, and Figure 5.3 (b) shows that this c-optimal design satisfies the equation (5.19). 


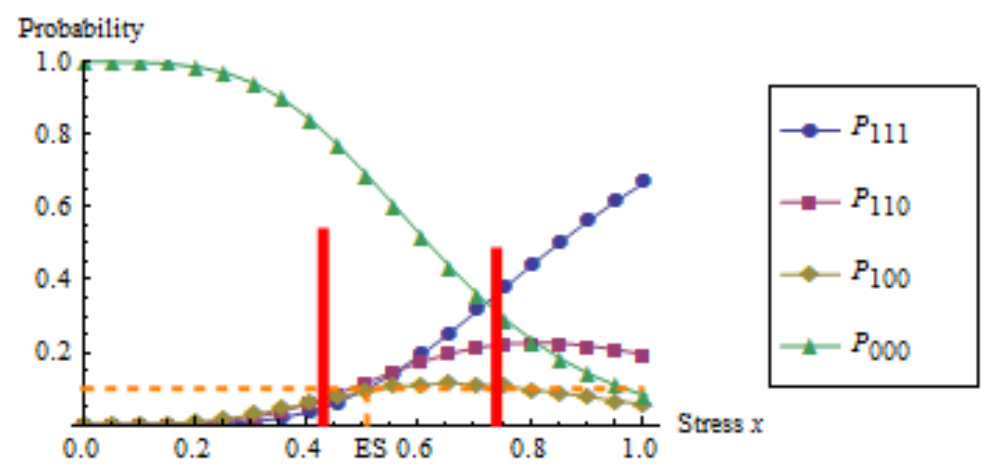

(a) c-Optimal design

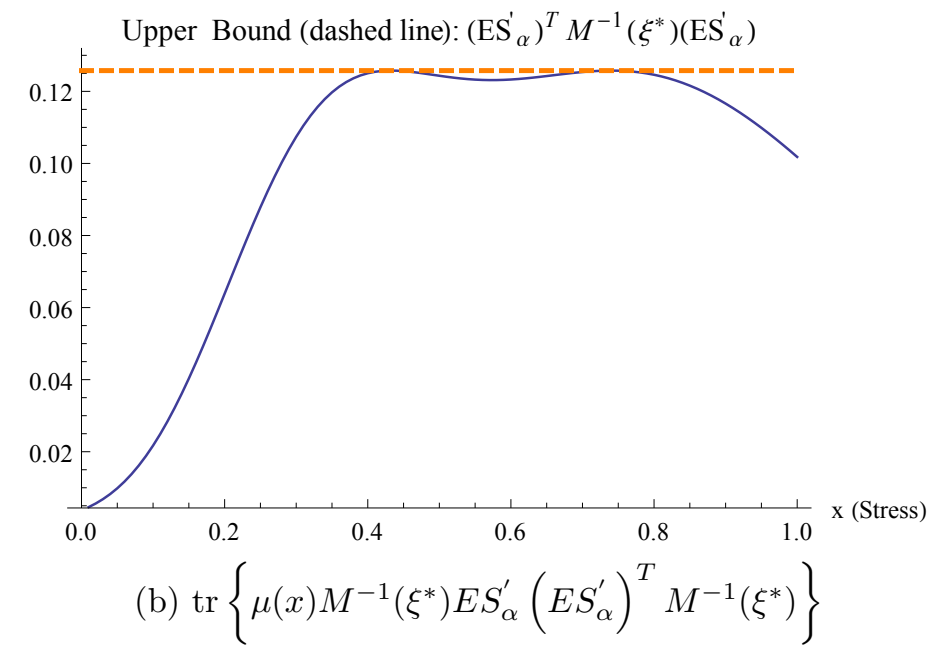

Figure 5.3: c-Optimal design for $c=E S_{\alpha=.1}$

**The candidate set of design points contains 100 equally spaced points within $(0,1]$. The vertical bars in (a) represent allocation proportions to stresses for the c-optimal design:

$$
\xi^{*}=\left\{\begin{array}{rr}
.43, & .74 \\
.5269, & .4731
\end{array}\right\} \text {. }
$$




\subsection{An Adaptive Optimal Design for Implementa- tion}

We used a two-stage procedure to find optimal designs with decreasing responses under the trivariate Weibull regression model.

Now two possible cases have been considered. Assume that the true parameter is $\boldsymbol{\theta}=\{-4.0,3.5,2.5,1.5,2\}$, and its locally optimal design is

$$
\xi_{\boldsymbol{\theta}}=\left\{\begin{array}{rr}
.43, & .74 \\
.5269, & .4731
\end{array}\right\} \text {. }
$$

In the first case, we used $\boldsymbol{\theta}_{01}=\{-4.2,3.3,2.7,1.7,1\}$ as the initial parameter in stage 1 of a two-stage design. The locally optimal design for the initial parameter $\boldsymbol{\theta}_{01}$ is

$$
\xi_{\boldsymbol{\theta}_{01}}=\left\{\begin{array}{rr}
.76, & .99 \\
.6111, & .3889
\end{array}\right\}
$$

the efficiency is $\left[\left(\left(E S_{\alpha}^{\prime}\right)^{T} M^{-1}\left(\xi_{\boldsymbol{\theta}}, \boldsymbol{\theta}\right) E S_{\alpha}^{\prime}\right) /\left(\left(E S_{\alpha}^{\prime}\right)^{T} M^{-1}\left(\xi_{\boldsymbol{\theta}_{01}}, \boldsymbol{\theta}\right) E S_{\alpha}^{\prime}\right)\right]=.1207$. This tells us that if the true parameter $\boldsymbol{\theta}$ is misspecified as $\boldsymbol{\theta}_{01}, 8.29(1 / .1207=8.29)$ times more samples are needed to produce the same accuracy for estimating the true parameter using $\xi_{\boldsymbol{\theta}_{01}}$ instead of $\xi_{\boldsymbol{\theta}}$. It shows that $\boldsymbol{\theta}_{01}$ is a very inefficient choice. Figure 5.4 (a) shows us the efficiency of two-stage adaptive optimal designs with various sample sizes $(\mathrm{n}=400,1,000$, and 4,000). Simulation steps are in Appendix A.14. Compared to the efficiency of the initial locally optimal design (dashed line), twostage adaptive optimal designs have substantially larger information. It demonstrates that one can improve efficiency by using two-stage adaptive optimal designs. 
In the second case, we used $\boldsymbol{\theta}_{02}=\{-3.6,3.7,2.3,1.5,2\}$ as the initial parameter. The locally optimal design for the initial parameter $\boldsymbol{\theta}_{02}$ is

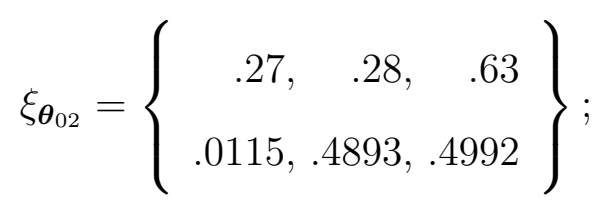

the efficiency is $\left[\left(\left(E S_{\alpha}^{\prime}\right)^{T} M^{-1}\left(\xi_{\boldsymbol{\theta}}, \boldsymbol{\theta}\right) E S_{\alpha}^{\prime}\right) /\left(\left(E S_{\alpha}^{\prime}\right)^{T} M^{-1}\left(\xi_{\boldsymbol{\theta}_{02}}, \boldsymbol{\theta}\right) E S_{\alpha}^{\prime}\right)\right]=.7840$. It tells us that if the true parameter $\boldsymbol{\theta}$ is misspecified as $\boldsymbol{\theta}_{02}, 1.28(1 / .7840=1.28)$ times more samples are needed to produce the same accuracy for estimating the true parameter using $\xi_{\boldsymbol{\theta}_{02}}$ instead of $\xi_{\boldsymbol{\theta}}$. In this case, $\boldsymbol{\theta}_{02}$ is pretty efficient choice. Figure 5.4 (b) shows us that two-stage adaptive optimal designs still have larger information than the initial locally optimal design. In conclusion, we can compensate for the loss of information from the initial misspecified parameters by using a two-stage procedure. 


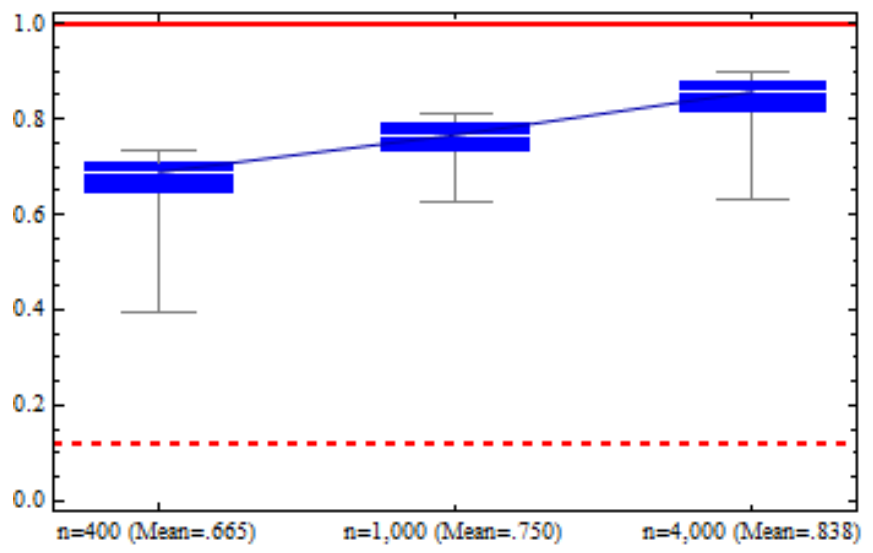

(a) Case 1

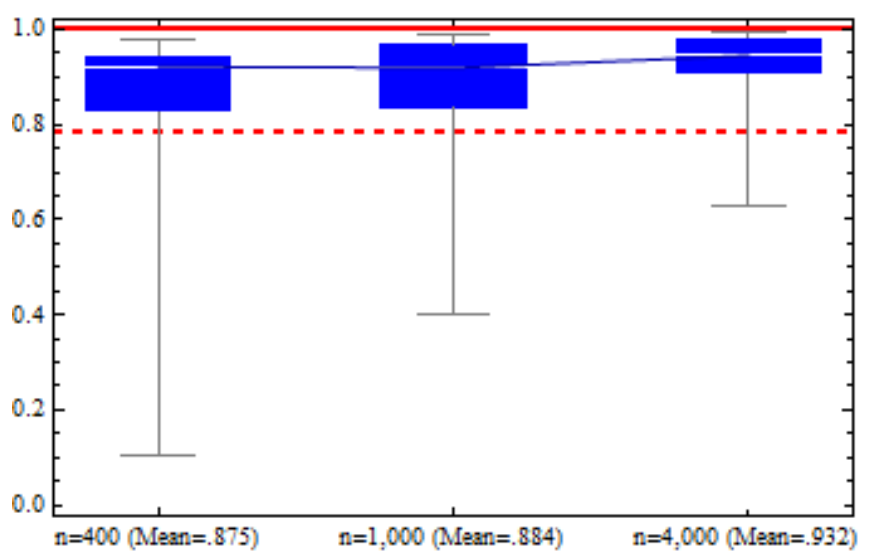

(b) Case 2

Figure 5.4: Boxplots of the efficiency of the two-stage adaptive optimal design relative to the initial locally optimal design $\xi_{\boldsymbol{\theta}_{0}}$. The efficiency of the locally optimal design $\xi_{\boldsymbol{\theta}_{0}}$ (used in the first stage of the adaptive procedure) is shown by the dashed line. $\xi_{\widehat{\boldsymbol{\theta}}_{0}}$ denotes a locally optimal design based on parameter estimates from the first stage design of $\xi_{\boldsymbol{\theta}_{0}}$.

$$
\begin{aligned}
& \text { Efficiency }=\left(\frac{\left(E S_{\alpha}^{\prime}\right)^{T} M^{-1}\left(\xi_{\boldsymbol{\theta}}, \boldsymbol{\theta}\right) E S_{\alpha}^{\prime}}{p_{0}\left(\left(E S_{\alpha}^{\prime}\right)^{T} M^{-1}\left(\xi_{\boldsymbol{\theta}_{0}}, \boldsymbol{\theta}\right) E S_{\alpha}^{\prime}\right)+\left(1-p_{0}\right)\left(\left(E S_{\alpha}^{\prime}\right)^{T} M^{-1}\left(\xi_{\widehat{\boldsymbol{\theta}}}, \boldsymbol{\theta}\right) E S_{\alpha}^{\prime}\right)}\right) \text { where } p_{0}=\frac{\sqrt{n}}{n}, \\
& \text { Dashed line: }\left(\frac{\left(E S_{\alpha}^{\prime}\right)^{T} M^{-1}\left(\xi_{\boldsymbol{\theta}}, \boldsymbol{\theta}\right) E S_{\alpha}^{\prime}}{\left(E S_{\alpha}^{\prime}\right)^{T} M^{-1}\left(\xi_{\boldsymbol{\theta}_{0}}, \boldsymbol{\theta}\right) E S_{\alpha}^{\prime}}\right) . \\
& \\
& \quad * * 100 \text { experiments have been performed for each } n .
\end{aligned}
$$




\section{Chapter 6}

\section{Future Studies}

\subsection{Multivariate Weibull Probability Density Func- tion}

A multivariate exponential probability density can be formed as an extension of Freund's bivariate exponential density. Let $R$ be a total number of random variables. Figure (6.1) shows the parameter structure given $t_{1}<t_{2} \cdots<t_{R}$ and the multivariate density is

$$
\begin{aligned}
f\left(t_{1}, t_{2}, \ldots, t_{R}\right)= & \left(\beta_{1} e^{-\beta_{1} t_{1}} e^{-\left(\beta_{2}+\beta_{3}+\cdots+\beta_{R}\right) t_{1}}\right) \times\left(\beta_{2 \mid 1} e^{-\beta_{2 \mid 1}\left(t_{2}-t_{1}\right)} e^{-\left(\beta_{3 \mid 1}+\beta_{4 \mid 1}+\cdots+\beta_{R \mid 1}\right)\left(t_{2}-t_{1}\right)}\right) \\
& \times \cdots \times \beta_{R \mid(R-1)(R-2) \cdots 1} e^{-\beta_{R \mid(R-1)(R-2) \cdots 1}\left(t_{R}-t_{R-1}\right)} \\
& \text { for } 0<t_{1}<t_{2}<\cdots<t_{R}<\infty .
\end{aligned}
$$


The density for other values of $t_{1}, t_{2}, \ldots, t_{R}$ is given in (A.19) of Appendix A.15.

A multivariate Weibull probability density is created from (6.1) using power transformations, $T_{1}=Y_{1}^{\tau}, T_{2}=Y_{2}^{\tau}, \ldots, T_{R}=Y_{R}^{\tau}$ with $\tau>0$ to obtain

$$
\begin{aligned}
f\left(y_{1}, y_{2}, \ldots, y_{R}\right)= & \left(\beta_{1} \beta_{2 \mid 1}+\cdots \beta_{R \mid(R-1)(R-2) \cdots 1} \tau^{R}\left(y_{1} y_{2} \cdots y_{R}\right)^{\tau-1}\right. \\
& \times e^{-\left(\beta_{1}+\beta_{2}+\cdots+\beta_{R}-\left(\beta_{2 \mid 1}+\beta_{3 \mid 1}+\cdots+\beta_{R \mid 1}\right)\right) y_{1}^{\tau}} \\
& \times e^{-\left(\beta_{2 \mid 1}+\beta_{3 \mid 1}+\cdots+\beta_{R \mid 1}-\left(\beta_{3 \mid 21}+\beta_{4 \mid 21}+\cdots+\beta_{R \mid 21}\right)\right) y_{2}^{\tau}} \times \cdots \times e^{-\beta_{R \mid(R-1)(R-2) \cdots 1} y_{R}^{\tau}} \\
& \text { for } 0<y_{1}<y_{2}<\cdots<y_{R}<\infty .
\end{aligned}
$$

The density for other values of $y_{1}, y_{2}, \ldots, y_{R}$ is given in (A.20) of Appendix A.16.

Assuming a system with deceasing amounts of damage, i.e, $\left(Y_{1}>Y_{2}>\cdots>\right.$ $Y_{R}$ ), and assuming a component can't fail before the previous components fail, the equation (5.6) can be extended to

$$
\begin{aligned}
g\left(y_{1}, y_{2}, \cdots, y_{R}\right)= & \left(\beta_{R} \beta_{(R-1) \mid R} \cdots \beta_{1 \mid 23 \cdots R}\right) \tau^{R}\left(y_{1} y_{2} \cdots y_{R}\right)^{\tau-1} \\
& \times e^{-\left(\beta_{R}-\beta_{(R-1) \mid R}\right) y_{R}^{\tau}-\left(\beta_{(R-1) \mid R}-\beta_{(R-2) \mid(R-1) R}\right) y_{(R-1)}^{\tau} \cdots-\beta_{1 \mid 23 \cdots R} y_{1}^{\tau}} \\
& \text { for } 0<y_{R}<y_{R-1}<\cdots<y_{1}<\infty .
\end{aligned}
$$

From (6.2), the probability of "system failure" can easily be computed:

$\mathrm{P}($ Failure for all components $)=\mathrm{P}\left(Y_{1}>v^{*}, Y_{2}>v^{*}, \cdots, Y_{R}>v^{*}\right)=\exp \left(-\beta_{R} v^{* \tau}\right)$.

With desired regression terms, one can compute an effective stress $E S_{\alpha}$ and find optimal designs. 


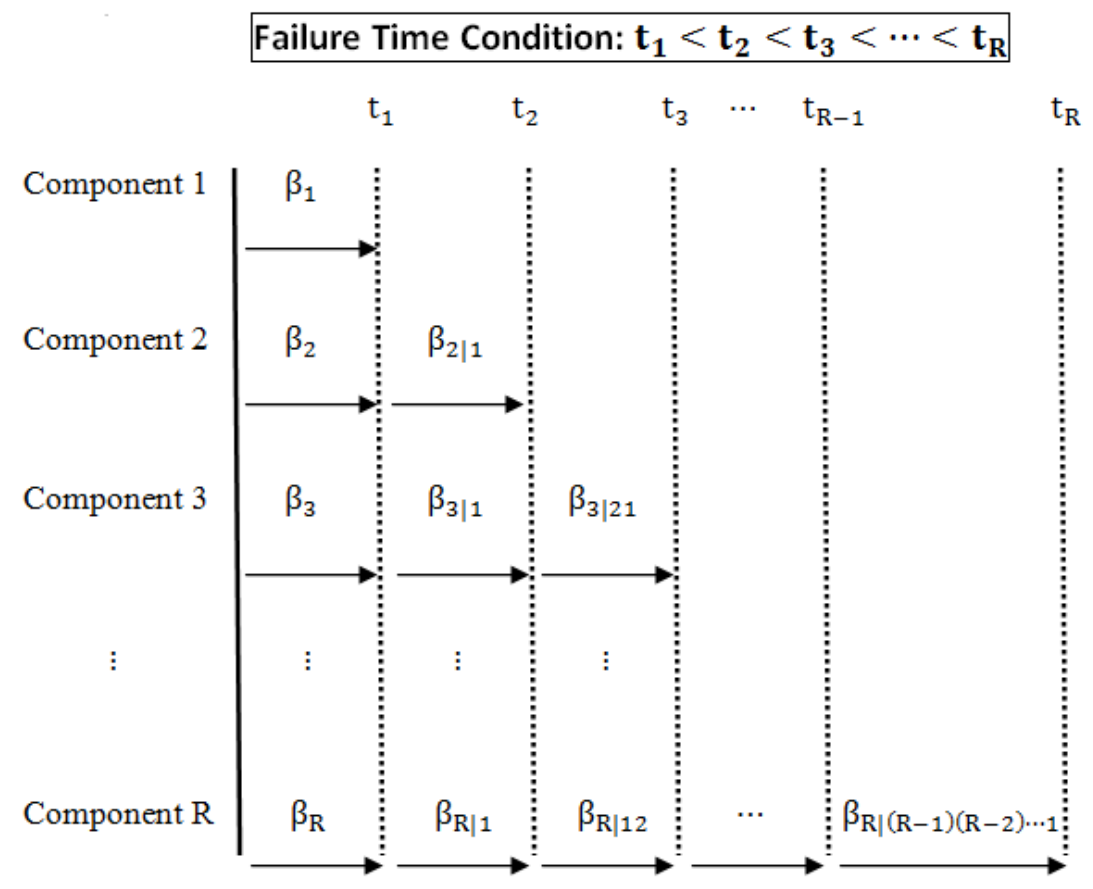

Figure 6.1: Failure rate change after component failures given $t_{1}<t_{2}<\cdots<t_{R}$ under a multivariate exponential model. 


\subsection{Optimal Designs for Serial and Parallel Sys- tems with Two Binary Outcomes}

Generally, in a serial system, a failure of any one component results in failure for the entire system. For example, a mobile phone may consists of the keypad, the ear piece, the microphone, and the display screen. One failure of any of these pieces will lead to failure in the entire system. In reliability studies, it is usual to assume the failures in each component are independent. However, it is easy to see dependences in serial systems. For example, a double block and bleed valve (Figure 6.2) has a sequential blocking system to isolate objects more safely from the outside stress. In this case, the first block failure doesn't mean failure for the entire system.

Let the first and the second blocks be the first and the second components respectively, then the amount of damage on the first component is always greater than the second component from the outside stress. Because the outside stress can reach to the second component after the first component has been broken. In mechanical systems, it is easy to see that amount of damage on one component is always greater than the other component given a stress like this example.

Define the blocking failures as the amount of damage are greater than predetermined cutoff values, and assume that there exists the dependency between two components such as the definition (6.3), 

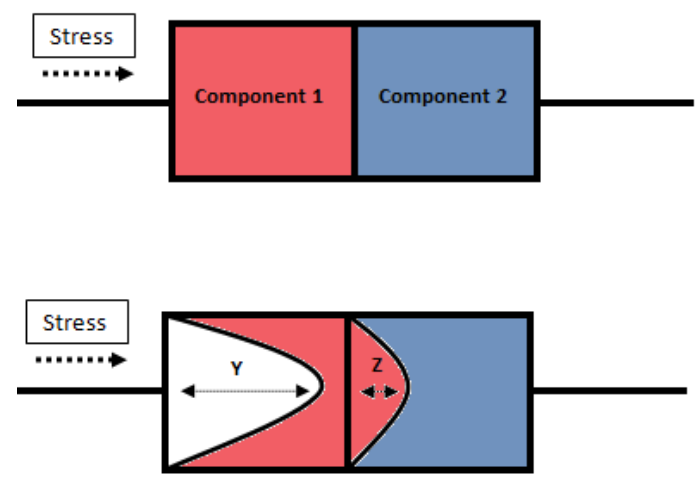

(a) Serial system

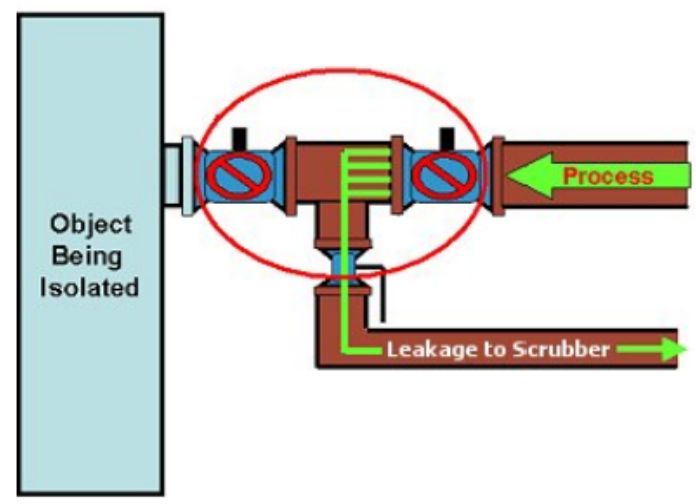

(b) Double block and bleed valve; chemvalve.com

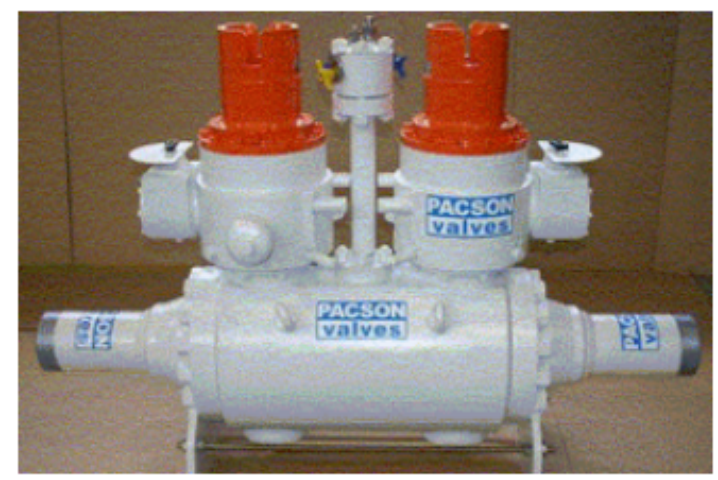

(c) The completed double block and bleed ball valve; www.controlengeurope.com

Figure 6.2: A serial system 


$$
\begin{aligned}
& U=\left\{\begin{array}{l}
0 \text { (No failure for the component } 1), \text { if } Y<v_{1}^{*} \\
1 \text { (Failure for the component } 1), \text { otherwise, }
\end{array}\right. \\
& V=\left\{\begin{array}{l}
0 \text { (No failure for the component } 2), \text { if } Z<v_{2}^{*} \\
1 \text { (Failure for the component } 2), \text { otherwise, }
\end{array}\right.
\end{aligned}
$$

where $Z<Y ; v_{1}^{*}$ and $v_{2}^{*}$ are predetermined cutoff values.

Then there is no possibility that the first component blocks the stress successfully, and the second component fails to block the stress. In other words, $p_{01}=\mathrm{P}($ Component 1 succeeds in blocking $\cap$ Component 2 fails to block $)=\mathrm{P}(U=0, V=1)$ must be zero

\begin{tabular}{|c|c|c|c|}
\hline $\begin{array}{ll}\text { 2nd Component } & \text { 1st Component }\end{array}$ & 0 (Success) & 1 (Failure) & \\
\hline 0 (Success) & $p_{00}$ & $p_{10}$ & $p_{0} 0$ \\
\hline 1 (Failure) & $p_{01}=0$ & $p_{11}$ & $p_{\cdot 1}$ \\
\hline & $p_{0}$ & $p_{1}$. & 1 \\
\hline
\end{tabular}
(see Table 6.1).

Table 6.1: Ternary outcome probabilities

Because if the first component blocks the outside stress successfully than the stress is unable to reach the second component. If we use a bivariate normal distribution model, this point is a problem, because there exists a $p_{01}=P\left(Y<v_{1}^{*}, Z>v_{2}^{*}\right)>\epsilon$ where $Y$ and $Z$ are bivariate normal random variables at any cutoff values $v_{1}^{*}$ and $v_{2}^{*}$ (Figure 6.3). However, this is not a problem in the bivariate Weibull distribution 
when we set $\beta_{1}=0$ in the equation (4.8). When $\beta_{1}=0$, the equation (4.8) becomes

$$
f^{*}(y, z)= \begin{cases}\beta_{2}\left(\beta_{4}+\beta_{3}\right) \tau^{2}(y z)^{\tau-1} e^{-\left(\beta_{4}+\beta_{3}\right) y^{\tau}-\left(\beta_{2}-\beta_{4}\right) z^{\tau}} & \text { for } 0<z<y<\infty \\ \beta_{3} \tau y^{\tau-1} e^{-\left(\beta_{2}+\beta_{3}\right) y^{\tau}} & \text { for } 0<y=z<\infty\end{cases}
$$

and the marginal density functions of the bivariate Weibull density with $\beta_{1}=0(6.4)$ are

$$
\begin{aligned}
& f^{*}(y)=(\alpha)\left(\beta_{4}+\beta_{3}\right) \tau y^{\tau-1} e^{-\left(\beta_{4}+\beta_{3}\right) y^{\tau}}+(1-\alpha)\left(\beta_{2}+\beta_{3}\right) \tau y^{\tau-1} e^{-\left(\beta_{2}+\beta_{3}\right) y^{\tau}} \\
& f^{*}(z)=\left(\beta_{2}+\beta_{3}\right) \tau y^{\tau-1} e^{-\left(\beta_{2}+\beta_{3}\right) y^{\tau}}
\end{aligned}
$$

where $\alpha=\left(\beta_{2} /\left(\beta_{2}-\beta_{4}\right)\right)$.

Since $\int_{A} f^{*}(y, z) d \mu=1$ where $A=\left\{\mathbf{w} \mid Z \leq Y, w_{1} \in Y\right.$ and $\left.w_{2} \in Z\right\}$ and $\mu$ is a probability measure, $f^{*}(y, z)$ is also a probability density function. In the model (6.4) where $Y$ and $Z$ are bivariate Weibull random variables, $p_{01}=P\left(Y<v_{1}^{*}, Z>v_{2}^{*}\right)$ is exactly zero at any $v_{1}^{*}$ and $v_{2}^{*}$ such that $v_{1}^{*} \leq v_{2}^{*}$ (Figure 6.4).

Other outcome probabilities can be defined:

$$
\begin{aligned}
& p_{00}=\int_{0}^{v_{1}^{*}} \int_{0}^{v_{2}^{*}} f^{*}(y, z) d y d z \\
& p_{10}=\int_{v_{1}^{*}}^{\infty} \int_{0}^{v_{2}^{*}} f^{*}(y, z) d z d y \\
& p_{11}=\int_{v_{1}^{*}}^{\infty} \int_{v_{2}^{*}}^{\infty} f^{*}(y, z) d y d z .
\end{aligned}
$$




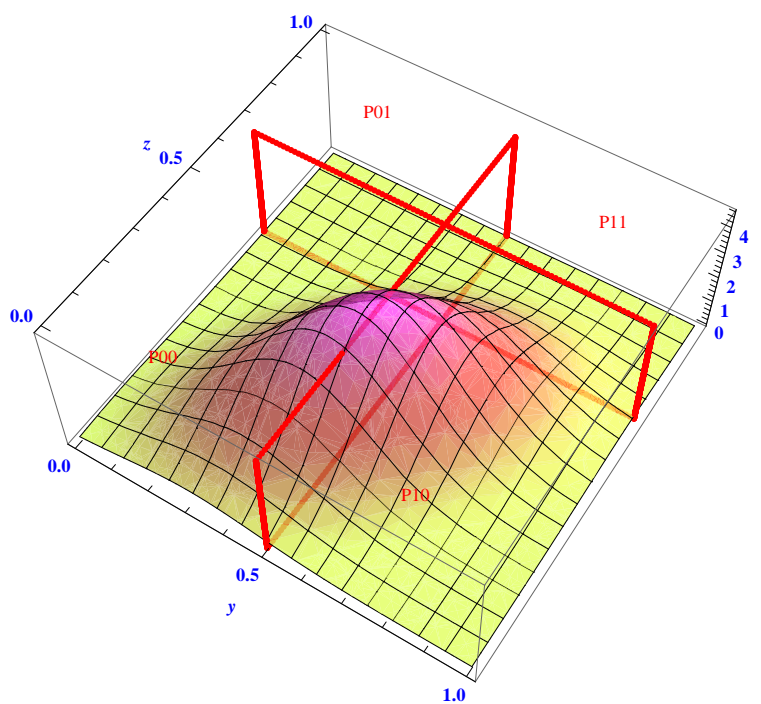

(a)

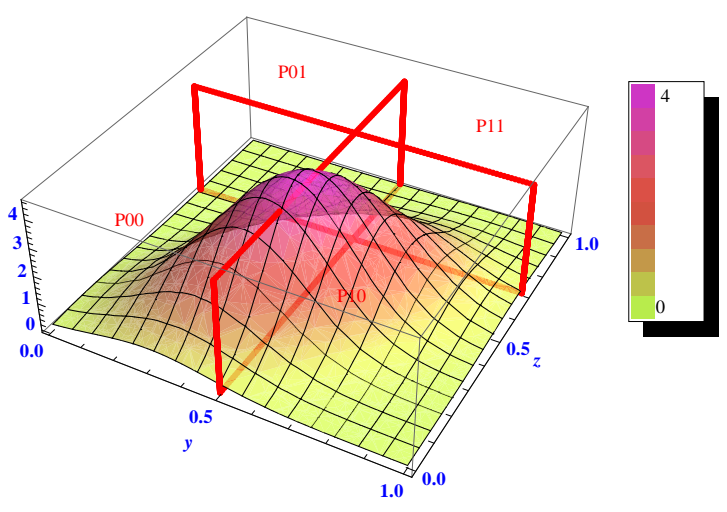

(b)

Figure 6.3: The bivariate normal distribution $\mu_{1}=0.5, \mu_{2}=0.4, \sigma_{1}=0.2, \sigma_{2}=0.2, \rho=0.5$. At any $v_{1}^{*}$ and $v_{2}^{*}, p_{01}>\epsilon$.

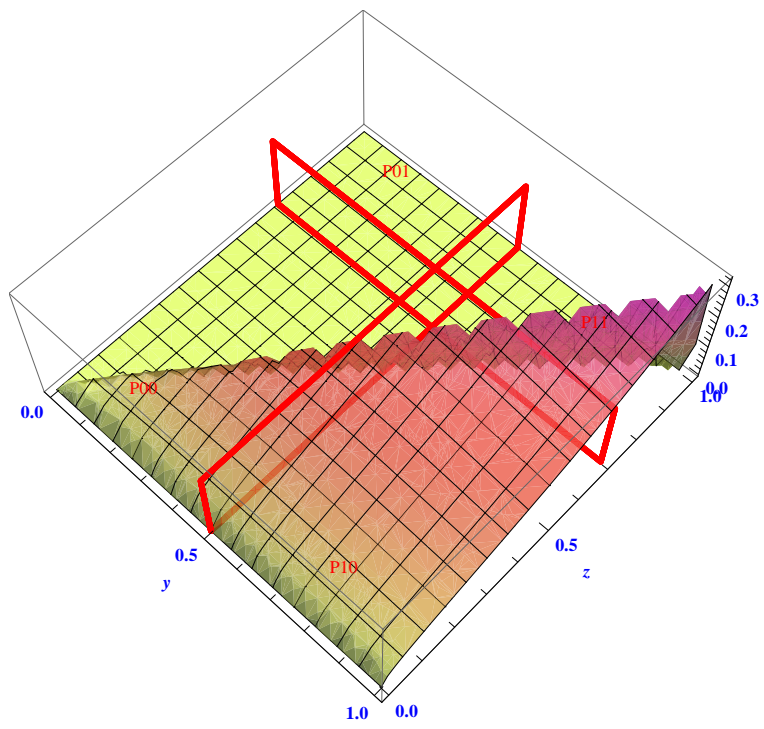

(a)

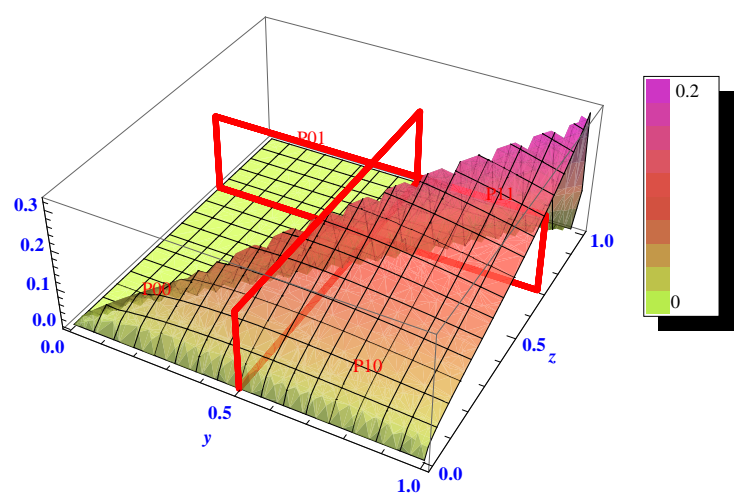

(b)

Figure 6.4: The bivariate Weibull distribution $\beta_{1}=0, \beta_{2}=0.2, \beta_{3}=.1, \beta_{4}=.9, \beta_{5}=.9, \tau=1.5$. $p_{01}=0$ when $\beta_{1}=0$ and $v_{1}^{*}<v_{2}^{*}$. 
In the bivariate Weibull distribution (4.8), we proposed the regression model by making connections between regression terms and the scale parameters:

$$
\begin{aligned}
& -\log \left(\beta_{5}+\beta_{3}\right)=\eta_{1}\left(\boldsymbol{x} ; \boldsymbol{\vartheta}_{1}\right) \\
& -\log \left(\beta_{4}+\beta_{3}\right)=\eta_{2}\left(\boldsymbol{x} ; \boldsymbol{\vartheta}_{2}\right) \\
& -\log \left(\beta_{1}+\beta_{2}+\beta_{3}\right)=\eta_{3}\left(\boldsymbol{x} ; \boldsymbol{\vartheta}_{3}\right),
\end{aligned}
$$

where $\eta_{1}=\eta_{1}\left(\boldsymbol{x} ; \boldsymbol{\vartheta}_{1}\right), \eta_{2}=\eta_{2}\left(\boldsymbol{x} ; \boldsymbol{\vartheta}_{2}\right)$, and $\eta_{3}=\eta_{3}\left(\boldsymbol{x} ; \boldsymbol{\vartheta}_{3}\right)$ are functions which in general may be nonlinear and $\boldsymbol{\vartheta}_{1}, \boldsymbol{\vartheta}_{2}$, and $\boldsymbol{\vartheta}_{3}$ may be vectors of parameters.

Similar to (6.6), the regression terms can be inserted in the model (6.4):

$$
\begin{aligned}
& -\log \left(\beta_{4}+\beta_{3}\right)=\eta_{1}\left(\boldsymbol{x} ; \boldsymbol{\vartheta}_{1}\right) \\
& -\log \left(\beta_{2}+\beta_{3}\right)=\eta_{2}\left(\boldsymbol{x} ; \boldsymbol{\vartheta}_{2}\right)
\end{aligned}
$$

Since scale parameters must be positive, it follows from (6.4) that the $\boldsymbol{\vartheta}_{1}$, and $\boldsymbol{\vartheta}_{2}$ must satisfy the conditions such that $\left(\beta_{2}-\beta_{4}\right)=e^{-\eta_{2}\left(\boldsymbol{x} ; \boldsymbol{\vartheta}_{2}\right)}-e^{-\eta_{1}\left(\boldsymbol{x} ; \boldsymbol{\vartheta}_{1}\right)}>0$ in the sample space.

Repalcing scale parameters $\left[\left(\beta_{4}+\beta_{3}\right)\right.$, and $\left.\left(\beta_{2}+\beta_{3}\right)\right]$ of Weibull density (6.4) with (6.7), $\beta_{3}$ is eliminated from the expression of the outcome probabilities in terms of regression parameters, but $\beta_{2}$ remains. Let $\boldsymbol{\theta}=\left\{\boldsymbol{\vartheta}_{1}, \boldsymbol{\vartheta}_{2}, \beta_{2}, \tau\right\}$. Then the loglikelihood function is

$$
l(\boldsymbol{\theta} \mid u, v ; \boldsymbol{x})=(u)(v) \ln \left(p_{11}\right)+(u)(1-v) \ln \left(p_{10}\right)+(1-u) \ln \left(1-p_{10}-p_{11}\right)
$$

the score function can be written as the product of three matrices: $\partial l(\boldsymbol{\theta} \mid u, v ; \boldsymbol{x}) / \partial \boldsymbol{\theta}=$ 
$A B C$, where

$$
\begin{gathered}
A=\left[\begin{array}{llll}
\frac{\partial \eta_{1}\left(\boldsymbol{x} ; \boldsymbol{\vartheta}_{1}\right)}{\partial \boldsymbol{\vartheta}_{1}} & \frac{\partial \eta_{2}\left(\boldsymbol{x} ; \boldsymbol{\vartheta}_{2}\right)}{\partial \boldsymbol{\vartheta}_{2}} & 1 & 1
\end{array}\right] \times \mathbf{1}_{2 \times 1}, \\
\frac{\partial \eta_{j}\left(\boldsymbol{x} ; \boldsymbol{\vartheta}_{j}\right)}{\partial \boldsymbol{\vartheta}_{j}}=\left[\begin{array}{llll}
\frac{\partial \eta_{j}\left(\boldsymbol{x} ; \boldsymbol{\vartheta}_{j}\right)}{\partial \vartheta_{j 1}} & \frac{\partial \eta_{j}\left(\boldsymbol{x} ; \boldsymbol{\vartheta}_{j}\right)}{\partial \vartheta_{j 2}} & \cdots & \frac{\partial \eta_{j}\left(\boldsymbol{x} ; \boldsymbol{\vartheta}_{j}\right)}{\partial \vartheta_{j m_{j}}}
\end{array}\right], j=1,2,
\end{gathered}
$$

$m_{j}$ is the number of parameters in $\boldsymbol{\theta}_{j}$ and $\mathbf{1}_{a \times b}$ is a $a \times b$ matrix of ones;

$$
B=\left[\begin{array}{cc}
\frac{\partial p_{11}}{\partial \eta_{1}\left(\boldsymbol{x} ; \boldsymbol{\vartheta}_{1}\right)} \times \mathbf{1}_{m_{1} \times 1} & \frac{\partial p_{10}}{\partial \eta_{1}\left(\boldsymbol{x} ; \boldsymbol{\vartheta}_{1}\right)} \times \mathbf{1}_{m_{1} \times 1} \\
\frac{\partial p_{11}}{\partial \eta_{2}\left(\boldsymbol{x} ; \boldsymbol{\vartheta}_{2}\right)} \times \mathbf{1}_{m_{2} \times 1} & \frac{\partial p_{10}}{\partial \eta_{2}\left(\boldsymbol{x} ; \boldsymbol{\vartheta}_{2}\right)} \times \mathbf{1}_{m_{2} \times 1} \\
\frac{\partial p_{11}}{\partial \beta_{2}} & \frac{\partial p_{10}}{\partial \beta_{2}} \\
\frac{\partial p_{11}}{\partial \tau} & \frac{\partial p_{10}}{\partial \tau}
\end{array}\right],
$$

Under standard regularity conditions, the information matrix can be written as

$$
\begin{aligned}
\mu(x, \boldsymbol{\theta}) & =E\left[\left(\frac{\partial l(\boldsymbol{\theta} \mid u, v ; \boldsymbol{x})}{\partial \boldsymbol{\theta}}\right)\left(\frac{\partial l(\boldsymbol{\theta} \mid u, v ; \boldsymbol{x})}{\partial \boldsymbol{\theta}}\right)^{T}\right]=E\left((A B C)(A B C)^{T}\right) \\
& =E\left(A B C C^{T} B^{T} A^{T}\right)=A B\left[E\left(C C^{T}\right)\right] B^{T} A^{T}=A B D B^{T} A^{T}
\end{aligned}
$$

where $D=E\left(C C^{T}\right)$. Since $U \sim \operatorname{Bernoulli}\left(p_{1}\right), V \sim \operatorname{Bernoulli}\left(p_{.1}\right)$ and $U$ and $V$ are 
dependent,

$$
\begin{aligned}
E\left(C C^{T}\right) & =\left[\begin{array}{cc}
\frac{1}{p_{11}}+\frac{1}{1-p_{10}-p_{11}} & \frac{1}{1-p_{10}-p_{11}} \\
\frac{1}{1-p_{10}-p_{11}} & \frac{1}{p_{10}}+\frac{1}{1-p_{10}-p_{11}}
\end{array}\right] \\
& =\operatorname{diag}\left(\frac{1}{p_{11}}, \frac{1}{p_{10}}\right)+\left(\frac{1}{1-p_{10}-p_{11}}\right) \mathbf{1}_{2 \times 2} .
\end{aligned}
$$

Assuming simple linear predictors with $\eta_{i}\left(\boldsymbol{x} ; \boldsymbol{\vartheta}_{i}\right)=\theta_{0}+\theta_{i} x$, the equation (6.7) becomes

$$
\begin{aligned}
& -\log \left(\beta_{4}+\beta_{3}\right)=\theta_{0}+\theta_{1} x \\
& -\log \left(\beta_{2}+\beta_{3}\right)=\theta_{0}+\theta_{2} x
\end{aligned}
$$

and the product of (6.8) and (6.9) becomes

$$
G^{T}=(A B)^{T}=\left[\begin{array}{lllll}
\frac{\partial p_{11}}{\partial \theta_{0}} & \frac{\partial p_{11}}{\partial \theta_{1}} & \frac{\partial p_{11}}{\partial \theta_{2}} & \frac{\partial p_{11}}{\partial \beta_{3}} & \frac{\partial p_{11}}{\partial \tau} \\
\frac{\partial p_{10}}{\partial \theta_{0}} & \frac{\partial p_{10}}{\partial \theta_{1}} & \frac{\partial p_{10}}{\partial \theta_{2}} & \frac{\partial p_{10}}{\partial \beta_{3}} & \frac{\partial p_{10}}{\partial \tau}
\end{array}\right]
$$

Based on this serial system, we will find D-optimal designs and c-optimal designs, then compare these optimal designs with optimal designs of parallel system. Then we will show two-stage adaptive designs. 


\section{Appendix A}

\section{A.1 Outcome Probabilities}

When $v_{1}^{*}>v_{2}^{*}$,

$$
\begin{aligned}
p_{11}(x \mid \boldsymbol{\theta})= & \frac{\beta_{2}}{\beta_{1}+\beta_{2}-\beta_{4}} e^{-\left(\beta_{4}+\beta_{3}\right) v_{1}^{* \tau}-\left(\beta_{1}+\beta_{2}-\beta_{4}\right) v_{2}^{* \tau}}+\frac{\beta_{1}-\beta_{4}}{\beta_{1}+\beta_{2}-\beta_{4}} e^{-\left(\beta_{1}+\beta_{2}+\beta_{3}\right) v_{1}^{* \tau}} ; \\
p_{10}(x \mid \boldsymbol{\theta})= & \frac{\beta_{2}}{\beta_{1}+\beta_{2}-\beta_{4}}\left(1-e^{\left.-\left(\beta_{1}+\beta_{2}-\beta_{4}\right) v_{2}^{* \tau}\right)} e^{-\left(\beta_{4}+\beta_{3}\right) v_{1}^{* \tau}} ;\right. \\
p_{01}(x \mid \boldsymbol{\theta})= & \frac{\beta_{2}}{\beta_{1}+\beta_{2}-\beta_{4}} e^{-\left(\beta_{1}+\beta_{2}-\beta_{4}\right) v_{2}^{* \tau}}\left(e^{-\left(\beta_{4}+\beta_{3}\right) v_{2}^{* \tau}}-e^{\left.-\left(\beta_{4}+\beta_{3}\right) v_{1}^{* \tau}\right)}\right. \\
& +\frac{\beta_{1}}{\beta_{1}+\beta_{2}-\beta_{5}}\left(1-e^{-\left(\beta_{1}+\beta_{2}-\beta_{5}\right) v_{2}^{* \tau}}\right) e^{-\left(\beta_{5}+\beta_{3}\right) v_{2}^{* \tau}} \\
& +\frac{\beta_{1}-\beta_{4}}{\beta_{1}+\beta_{2}-\beta_{4}}\left(e^{-\left(\beta_{1}+\beta_{2}+\beta_{3}\right) v_{2}^{* \tau}}-e^{-\left(\beta_{1}+\beta_{2}+\beta_{3}\right) v_{1}^{* \tau}}\right) ;
\end{aligned}
$$




$$
\begin{aligned}
p_{00}(x \mid \boldsymbol{\theta})= & \frac{\beta_{1}}{\beta_{1}+\beta_{2}-\beta_{5}}\left(1-e^{-\left(\beta_{5}+\beta_{3}\right) v_{2}^{* \tau}}\right) \\
& -\frac{\beta_{2}}{\beta_{1}+\beta_{2}-\beta_{4}}\left(1-e^{-\left(\beta_{1}+\beta_{2}-\beta_{4}\right) v_{2}^{* \tau}}\right) e^{-\left(\beta_{4}+\beta_{3}\right) v_{1}^{* \tau}} \\
& +\frac{\beta_{2}-\beta_{5}}{\beta_{1}+\beta_{2}-\beta_{5}}\left(1-e^{-\left(\beta_{1}+\beta_{2}+\beta_{3}\right) v_{2}^{* \tau}}\right) .
\end{aligned}
$$




\section{A.2 Outcome Probabilities with Predictor Func- tions (Equation (4.10))}

$$
\begin{aligned}
& -\log \left(\beta_{5}+\beta_{3}\right)=\eta_{1}\left(\boldsymbol{x} ; \boldsymbol{\vartheta}_{1}\right) \\
& -\log \left(\beta_{4}+\beta_{3}\right)=\eta_{2}\left(\boldsymbol{x} ; \boldsymbol{\vartheta}_{2}\right) \\
& -\log \left(\beta_{1}+\beta_{2}+\beta_{3}\right)=\eta_{3}\left(\boldsymbol{x} ; \boldsymbol{\vartheta}_{3}\right) .
\end{aligned}
$$

When $v_{1}^{*}>v_{2}^{*}$,

$$
\begin{aligned}
p_{11}(x \mid \boldsymbol{\theta})= & \frac{\beta_{2}}{e^{-\eta_{3}\left(\boldsymbol{x} ; \boldsymbol{\vartheta}_{3}\right)}-e^{-\eta_{2}\left(\boldsymbol{x} ; \boldsymbol{\vartheta}_{2}\right)}} \exp \left(-\left(e^{-\eta_{2}\left(\boldsymbol{x} ; \boldsymbol{\vartheta}_{2}\right)}\right) v_{1}^{* \tau}-\left(e^{-\eta_{3}\left(\boldsymbol{x} ; \boldsymbol{\vartheta}_{3}\right)}-e^{-\eta_{2}\left(\boldsymbol{x} ; \boldsymbol{\vartheta}_{2}\right)}\right) v_{2}^{* \tau}\right) \\
& +\frac{e^{-\eta_{3}\left(\boldsymbol{x} ; \boldsymbol{\vartheta}_{3}\right)}-e^{-\eta_{2}\left(\boldsymbol{x} ; \boldsymbol{\vartheta}_{2}\right)}-\beta_{2}}{e^{-\eta_{3}\left(\boldsymbol{x} ; \boldsymbol{\vartheta}_{3}\right)}-e^{-\eta_{2}\left(\boldsymbol{x} ; \boldsymbol{\vartheta}_{2}\right)}} \exp \left(-\left(e^{-\eta_{3}\left(\boldsymbol{x} ; \boldsymbol{\vartheta}_{3}\right)}\right) v_{1}^{* \tau}\right) ; \\
p_{10}(x \mid \boldsymbol{\theta})= & \frac{\beta_{2}}{e^{-\eta_{3}\left(\boldsymbol{x} ; \boldsymbol{\vartheta}_{3}\right)}-e^{-\eta_{2}\left(\boldsymbol{x} ; \boldsymbol{\vartheta}_{2}\right)}}\left(1-\exp \left(-\left(e^{-\eta_{3}\left(\boldsymbol{x} ; \boldsymbol{\vartheta}_{3}\right)}-e^{-\eta_{2}\left(\boldsymbol{x} ; \boldsymbol{\vartheta}_{2}\right)}\right) v_{2}^{* \tau}\right)\right) \\
& \times \exp \left(-\left(e^{-\eta_{2}\left(\boldsymbol{x} ; \boldsymbol{\vartheta}_{2}\right)}\right) v_{1}^{* \tau}\right) ;
\end{aligned}
$$




$$
\begin{aligned}
& p_{01}(x \mid \boldsymbol{\theta})=\frac{\beta_{2}}{e^{-\eta_{3}\left(\boldsymbol{x} ; \boldsymbol{\vartheta}_{3}\right)}-e^{-\eta_{2}\left(\boldsymbol{x} ; \boldsymbol{\vartheta}_{2}\right)}} \exp \left(-\left(e^{-\eta_{3}\left(\boldsymbol{x} ; \boldsymbol{\vartheta}_{3}\right)}-e^{-\eta_{2}\left(\boldsymbol{x} ; \boldsymbol{\vartheta}_{2}\right)}\right) v_{2}^{* \tau}\right) \\
& \times\left(\exp \left(-\left(e^{-\eta_{2}\left(\boldsymbol{x} ; \boldsymbol{\vartheta}_{2}\right)}\right) v_{2}^{* \tau}\right)-\exp \left(-\left(e^{-\eta_{2}\left(\boldsymbol{x} ; \boldsymbol{\vartheta}_{2}\right)}\right) v_{1}^{* \tau}\right)\right) \\
& +\frac{\beta_{1}}{e^{-\eta_{3}\left(\boldsymbol{x} ; \boldsymbol{\vartheta}_{3}\right)}-e^{-\eta_{1}\left(\boldsymbol{x} ; \boldsymbol{\vartheta}_{1}\right)}}\left(1-\exp \left(-\left(e^{-\eta_{3}\left(\boldsymbol{x} ; \boldsymbol{\vartheta}_{3}\right)}-e^{-\eta_{1}\left(\boldsymbol{x} ; \boldsymbol{\vartheta}_{1}\right)}\right) v_{2}^{* \tau}\right)\right) \\
& \times \exp \left(-\left(e^{-\eta_{1}\left(\boldsymbol{x} ; \boldsymbol{\vartheta}_{1}\right)}\right) v_{2}^{* \tau}\right) \\
& +\frac{e^{-\eta_{3}\left(\boldsymbol{x} ; \boldsymbol{\vartheta}_{3}\right)}-e^{-\eta_{2}\left(\boldsymbol{x} ; \boldsymbol{\vartheta}_{2}\right)}-\beta_{2}}{e^{-\eta_{3}\left(\boldsymbol{x} ; \boldsymbol{\vartheta}_{3}\right)}-e^{-\eta_{2}\left(\boldsymbol{x} ; \boldsymbol{\vartheta}_{2}\right)}}\left(\exp \left(-\left(e^{-\eta_{3}\left(\boldsymbol{x} ; \boldsymbol{\vartheta}_{3}\right)}\right) v_{2}^{* \tau}\right)-\exp \left(-\left(e^{-\eta_{3}\left(\boldsymbol{x} ; \boldsymbol{\vartheta}_{3}\right)}\right) v_{1}^{* \tau}\right)\right) \\
& p_{00}(x \mid \boldsymbol{\theta})=\frac{\beta_{1}}{e^{-\eta_{3}\left(\boldsymbol{x} ; \boldsymbol{\vartheta}_{3}\right)}-e^{-\eta_{1}\left(\boldsymbol{x} ; \boldsymbol{\vartheta}_{1}\right)}}\left(1-\exp \left(-\left(e^{-\eta_{1}\left(\boldsymbol{x} ; \boldsymbol{\vartheta}_{1}\right)}\right) v_{2}^{* \tau}\right)\right) \\
& -\frac{\beta_{2}}{e^{-\eta_{3}\left(\boldsymbol{x} ; \boldsymbol{\vartheta}_{3}\right)}-e^{-\eta_{2}\left(\boldsymbol{x} ; \boldsymbol{\vartheta}_{2}\right)}}\left(1-\exp \left(-\left(e^{-\eta_{3}\left(\boldsymbol{x} ; \boldsymbol{\vartheta}_{3}\right)}-e^{-\eta_{2}\left(\boldsymbol{x} ; \boldsymbol{\vartheta}_{2}\right)}\right) v_{2}^{* \tau}\right)\right) \\
& \times \exp \left(-\left(e^{-\eta_{2}\left(\boldsymbol{x} ; \boldsymbol{\vartheta}_{2}\right)}\right) v_{1}^{* \tau}\right) \\
& +\frac{e^{-\eta_{3}\left(\boldsymbol{x} ; \boldsymbol{\vartheta}_{3}\right)}-e^{-\eta_{1}\left(\boldsymbol{x} ; \boldsymbol{\vartheta}_{1}\right)}-\beta_{1}}{e^{-\eta_{3}\left(\boldsymbol{x} ; \boldsymbol{\vartheta}_{3}\right)}-e^{-\eta_{1}\left(\boldsymbol{x} ; \boldsymbol{\vartheta}_{1}\right)}}\left(1-\exp \left(-\left(e^{-\eta_{3}\left(\boldsymbol{x} ; \boldsymbol{\vartheta}_{3}\right)}\right) v_{2}^{* \tau}\right)\right) .
\end{aligned}
$$




\section{A.3 Outcome Probabilities with Linear Predictor Functions (Equation (4.20))}

$$
\begin{aligned}
& -\log \left(\beta_{5}+\beta_{3}\right)=\theta_{0}+\theta_{1} x \\
& -\log \left(\beta_{4}+\beta_{3}\right)=\theta_{0}+\theta_{2} x \\
& -\log \left(\beta_{1}+\beta_{2}+\beta_{3}\right)=\theta_{0}+\theta_{3} x .
\end{aligned}
$$

When $v_{1}^{*}>v_{2}^{*}$,

$$
\begin{aligned}
p_{11}(x \mid \boldsymbol{\theta})= & \frac{\beta_{2}}{e^{-\left(\theta_{0}+\theta_{3} x\right)}-e^{-\left(\theta_{0}+\theta_{2} x\right)}} \exp \left(-\left(e^{-\left(\theta_{0}+\theta_{2} x\right)}\right) v_{1}^{* \tau}-\left(e^{-\left(\theta_{0}+\theta_{3} x\right)}-e^{-\left(\theta_{0}+\theta_{2} x\right)}\right) v_{2}^{* \tau}\right) \\
& +\frac{e^{-\left(\theta_{0}+\theta_{3} x\right)}-e^{-\left(\theta_{0}+\theta_{2} x\right)}-\beta_{2}}{e^{-\left(\theta_{0}+\theta_{3} x\right)}-e^{-\left(\theta_{0}+\theta_{2} x\right)}} \exp \left(-\left(e^{-\left(\theta_{0}+\theta_{3} x\right)}\right) v_{1}^{* \tau}\right) ; \\
p_{10}(x \mid \boldsymbol{\theta})= & \frac{\beta_{2}}{e^{-\left(\theta_{0}+\theta_{3} x\right)}-e^{-\left(\theta_{0}+\theta_{2} x\right)}}\left(1-\exp \left(-\left(e^{-\left(\theta_{0}+\theta_{3} x\right)}-e^{-\left(\theta_{0}+\theta_{2} x\right)}\right) v_{2}^{* \tau}\right)\right) \\
& \times \exp \left(-\left(e^{-\left(\theta_{0}+\theta_{2} x\right)}\right) v_{1}^{* \tau}\right)
\end{aligned}
$$




$$
\begin{aligned}
& p_{01}(x \mid \boldsymbol{\theta})=\frac{\beta_{2}}{e^{-\left(\theta_{0}+\theta_{3} x\right)}-e^{-\left(\theta_{0}+\theta_{2} x\right)}} \exp \left(-\left(e^{-\left(\theta_{0}+\theta_{3} x\right)}-e^{-\left(\theta_{0}+\theta_{2} x\right)}\right) v_{2}^{* \tau}\right) \\
& \times\left(\exp \left(-\left(e^{-\left(\theta_{0}+\theta_{2} x\right)}\right) v_{2}^{* \tau}\right)-\exp \left(-\left(e^{-\left(\theta_{0}+\theta_{2} x\right)}\right) v_{1}^{* \tau}\right)\right) \\
& \left.+\frac{\beta_{1}}{e^{-\left(\theta_{0}+\theta_{3} x\right)}-e^{-\left(\theta_{0}+\theta_{1} x\right)}}\left(1-\exp \left(-\left(e^{-\left(\theta_{0}+\theta_{3} x\right)}-e^{-\left(\theta_{0}+\theta_{1} x\right)}\right) v_{2}^{* \tau}\right)\right)\right) \\
& \times \exp \left(-\left(e^{-\left(\theta_{0}+\theta_{1} x\right)}\right) v_{2}^{* \tau}\right) \\
& +\frac{e^{-\left(\theta_{0}+\theta_{3} x\right)}-e^{-\left(\theta_{0}+\theta_{2} x\right)}-\beta_{2}}{e^{-\left(\theta_{0}+\theta_{3} x\right)}-e^{-\left(\theta_{0}+\theta_{2} x\right)}}\left(\exp \left(-\left(e^{-\left(\theta_{0}+\theta_{3} x\right)}\right) v_{2}^{* \tau}\right)-\exp \left(-\left(e^{-\left(\theta_{0}+\theta_{3} x\right)}\right) v_{1}^{* \tau}\right)\right) \\
& p_{00}(x \mid \boldsymbol{\theta})=\frac{\beta_{1}}{e^{-\left(\theta_{0}+\theta_{3} x\right)}-e^{-\left(\theta_{0}+\theta_{1} x\right)}}\left(1-\exp \left(-\left(e^{-\left(\theta_{0}+\theta_{1} x\right)}\right) v_{2}^{* \tau}\right)\right) \\
& \left.-\frac{\beta_{2}}{e^{-\left(\theta_{0}+\theta_{3} x\right)}-e^{-\left(\theta_{0}+\theta_{2} x\right)}}\left(1-\exp \left(-\left(e^{-\left(\theta_{0}+\theta_{3} x\right)}-e^{-\left(\theta_{0}+\theta_{2} x\right)}\right) v_{2}^{* \tau}\right)\right)\right) \\
& \times \exp \left(-\left(e^{-\left(\theta_{0}+\theta_{2} x\right)}\right) v_{1}^{* \tau}\right) \\
& +\frac{e^{-\left(\theta_{0}+\theta_{3} x\right)}-e^{-\left(\theta_{0}+\theta_{1} x\right)}-\beta_{1}}{e^{-\left(\theta_{0}+\theta_{3} x\right)}-e^{-\left(\theta_{0}+\theta_{1} x\right)}}\left(1-\exp \left(-\left(e^{-\left(\theta_{0}+\theta_{3} x\right)}\right) v_{2}^{* \tau}\right)\right) .
\end{aligned}
$$




\section{A.4 A Proof of the Equivalent Conditions for the D-Optimal Criterion (Chapter 4.2.5)}

Let $M(\xi)$ be the $(m \times m)$ information matrix of the design $\xi$ where $m$ is the number

of parameters, $\bar{\xi}$ be a design with unit weight at the point $x$, and the updated design $\xi^{\prime}$ be

$$
\xi^{\prime}=(1-\alpha) \xi+\alpha \bar{\xi}, 0 \leq \alpha \leq 1
$$

Then the updated information matrix of $\xi^{\prime}$ is

$$
M\left(\xi^{\prime}\right)=(1-\alpha) M(\xi)+\alpha M(\bar{\xi})
$$

Consider the directive derivative of $\Psi$ in the direction of $\bar{\xi}$ :

$$
\lim _{\alpha \rightarrow 0+} \frac{[\Psi\{(1-\alpha) M(\xi)+\alpha M(\bar{\xi})\}-\Psi\{M(\xi)\}]}{\alpha} .
$$

Since the equation (A.1) is a singular, 


$$
\begin{aligned}
& \lim _{\alpha \rightarrow 0+} \frac{[\Psi\{(1-\alpha) M(\xi)+\alpha M(\bar{\xi})\}-\Psi\{M(\xi)\}]}{\alpha} \\
& =\operatorname{tr}\left(\lim _{\alpha \rightarrow 0+} \frac{[\Psi\{(1-\alpha) M(\xi)+\alpha M(\bar{\xi})\}-\Psi\{M(\xi)\}]}{\alpha}\right) \\
& =\operatorname{tr}\left(\lim _{\alpha \rightarrow 0+} \frac{[\Psi\{M(\xi)+\alpha(M(\bar{\xi})-M(\xi))\}-\Psi\{M(\xi)\}]}{\alpha}\right) \\
& =\operatorname{tr}\left(\lim _{\alpha \rightarrow 0+} \frac{[\Psi\{M(\xi)+\alpha(M(\bar{\xi})-M(\xi))\}-\Psi\{M(\xi)\}]}{\alpha(M(\bar{\xi})-M(\xi))}(M(\bar{\xi})-M(\xi))\right) \\
& =\operatorname{tr}\left(\frac{\partial \Psi\{M(\xi)\}}{\partial M(\xi)}(M(\bar{\xi})-M(\xi))\right) \\
& =\operatorname{tr}\left(\frac{\partial \Psi\{M(\xi)\}}{\partial M(\xi)} M(\bar{\xi})\right)-\operatorname{tr}\left(\frac{\partial \Psi\{M(\xi)\}}{\partial M(\xi)} M(\xi)\right) .
\end{aligned}
$$

If we assume

$$
\Psi\{M(\xi)\}=\log \left|M(\xi)^{-1}\right|
$$

then

$$
\frac{\partial \Psi\{M(\xi)\}}{\partial M(\xi)}=-\left(M(\xi)^{-1}\right)^{T}
$$

Inserting (A.4) into (A.2), then (A.2) becomes

$$
\begin{gathered}
\operatorname{tr}\left(-M(\xi)^{-1} M(\bar{\xi})\right)+\operatorname{tr}\left(M(\xi)^{-1} M(\xi)\right) \\
=m-\operatorname{tr}\left(M(\xi)^{-1} M(\bar{\xi})\right) .
\end{gathered}
$$

Since $\Psi$ in (A.3) is a convex function of $M(\xi)$, (A.5) must be positive at any $M(\xi)$, and it has a minimum $M(\xi)$ at the equation $(\mathrm{A} .5)=0$ :

$$
\operatorname{tr}\left(M(\xi)^{-1} M(\bar{\xi})\right) \leq m
$$


Replacing $M(\bar{\xi})$ to $\mu(x)$, then (A.6) becomes

$$
\operatorname{tr}\left(\mu(x) M(\xi)^{-1}\right) \leq m
$$

It is the end of proof of the equivalent conditions for D-optimal criterion. 


\section{A.5 The Exchange Algorithm for D-Optimality}

Step 1. Find an initial design $\xi_{s_{0}}=\left\{x_{i}, w_{i}\right\}_{1}^{K}$ where $w_{i}=n_{i} / s_{0}$ and $s_{0}=\sum_{1}^{K} n_{i}$ that provides a regular average information matrix for design $\xi_{s_{0}}$ (see Chapter 4.2.2).

Step 2. Based on a design $\xi_{s}$, find the next design point such that

$$
x_{s}=\arg \max \left[d\left(x_{s}^{+}, \xi_{s}\right)-m, m-d\left(x_{s}^{-}, \xi_{s}\right)\right],
$$

where

$$
\begin{gathered}
d\left(x, \xi_{s}, \boldsymbol{\theta}\right)=\operatorname{tr}\left\{\mu(x, \boldsymbol{\theta}) M^{-1}\left(\xi_{s}, \boldsymbol{\theta}\right)\right\} \\
\left\{\begin{array}{l}
x_{s}^{+}=\arg \max _{x \in X} d\left(x, \xi_{s}, \boldsymbol{\theta}\right) \\
x_{s}^{-}=\arg \min _{x \in X_{s}} d\left(x, \xi_{s}, \boldsymbol{\theta}\right)
\end{array}\right.
\end{gathered}
$$

$X$ is the set of candidate points, $X_{s}$ is the set of support points in $\xi_{s}$, and $m=\operatorname{dim}(\boldsymbol{\theta})$. We use a candidate set that contains 100 equally spaced points within $(0,1]$.

Step 3. Add $x_{s}$ to the design $\xi_{s}$, to make a new design $\xi_{s+1}$ such that

$$
\xi_{s+1}=\left(1-\alpha_{s}\right) \xi_{s}+\alpha_{s} \xi\left(x_{s}\right)
$$


where $\xi\left(x_{s}\right)$ is a design at the point $x_{s}$ with weight 1 , i.e., $\xi\left(x_{s}\right)=\left\{\begin{array}{c}x_{s} \\ 1\end{array}\right\}$, and $\alpha_{s}$ (in this paper) is simply defined as

$$
\alpha_{s}= \begin{cases}\frac{1}{s+1}, & \text { if } x_{s}=x_{s}^{+} \\ -\min \left(\frac{1}{s+1}, \frac{w_{s}^{-}}{1-w_{s}^{-}}\right) & \text {if } x_{s}=x_{s}^{-}\end{cases}
$$

where $w_{s}^{-}$is the weight of $x_{s}^{-}$in the design $\xi_{s}$.

Steps 2 and 3 add new good design points to a previous design or put more weight on good design points. Otherwise, they delete bad design points or reduce the weight on bad design points.

Step 4. Iterate steps 2-3 until

$$
\left|d\left(x_{s}, \xi_{s}, \boldsymbol{\theta}\right)-m\right| \leq \epsilon
$$

where $m=\operatorname{dim}(\boldsymbol{\theta})$, or until a predetermined total number of iterations is reached which is determined by researcher. We set $\epsilon=.001$, and the maximum number of iteration is 5,000.

Note that to estimate parameters in a two-stage adaptive optimal design, the average information matrix must be full rank. The average information matrix with linear predictor functions (see Chapter 4.3) requires at least 3 design points to be full rank. Therefore, we don't reduce the number of design points before 
three.

Theoretically, for an optimal design $\xi^{*}, d\left(x, \xi^{*}, \boldsymbol{\theta}\right)$ can't be larger than $m$, and $d\left(x, \xi^{*}, \boldsymbol{\theta}\right)=m$ at all support points [Kiefer-Wolfowitz's Equivalence theorem (1974)]. We plotted $d\left(x, \xi^{*}, \boldsymbol{\theta}\right)$ for all designs found by the exchange algorithm to verify that it did, indeed, find the optimal design.

To find a penalized locally D-optimal design, (A.8) is replaced by

$$
d\left(x, \xi_{s}, \boldsymbol{\theta}\right)=\phi^{-1}(x, \boldsymbol{\theta}) \operatorname{tr}\left\{\mu(x, \boldsymbol{\theta}) M^{-1}\left(\xi_{s}, \boldsymbol{\theta}\right)\right\},
$$

and (A.9) is replaced by

$$
\begin{gathered}
\left|d\left(x_{s}, \xi_{s}, \boldsymbol{\theta}\right)-\Phi^{-1}\left(\xi_{s}, \boldsymbol{\theta}\right) m\right| \leq \epsilon \\
\Rightarrow\left|\phi^{-1}\left(x_{s}, \boldsymbol{\theta}\right) \operatorname{tr}\left\{\mu\left(x_{s}, \boldsymbol{\theta}\right) M^{-1}\left(\xi_{s}, \boldsymbol{\theta}\right)\right\}-\Phi^{-1}\left(\xi_{s}, \boldsymbol{\theta}\right) m\right| \leq \epsilon .
\end{gathered}
$$




\section{A.6 Simulation Study I}

Let the bivariate Weibull density (4.8) containing (4.20) be $f(y, z \mid x, \boldsymbol{\theta})$ :

$$
f(y, z \mid x, \boldsymbol{\theta})= \begin{cases}f_{1}(y, z \mid x, \boldsymbol{\theta}) & \text { for } 0<y<z<\infty \\ f_{2}(y, z \mid x, \boldsymbol{\theta}) & \text { for } 0<z<y<\infty \\ f_{3}(y, z \mid x, \boldsymbol{\theta}) & \text { for } 0<y=z<\infty\end{cases}
$$

Define conditions (1), (2), and (3) to be $0<y<z<\infty, 0<z<y<\infty$, and $0<y=z<\infty$ respectively. Define the marginal density functions of $Y$ and $Z$ given the conditions (1), (2), and (3) to be $f_{Y q}(y \mid x, \boldsymbol{\theta})$ and $f_{Z q}(z \mid x, \boldsymbol{\theta})$ where $q=1,2,3$, respectively.

Let the cumulative sub-distributions be $F(y, z \mid x, \boldsymbol{\theta})$ :

$$
F(y, z \mid x, \boldsymbol{\theta})= \begin{cases}F_{1}(y, z \mid x, \boldsymbol{\theta}) & \text { for } 0<y<z<\infty \\ F_{2}(y, z \mid x, \boldsymbol{\theta}) & \text { for } 0<z<y<\infty \\ F_{3}(y, z \mid x, \boldsymbol{\theta}) & \text { for } 0<y=z<\infty\end{cases}
$$

and denote the marginal cumulative sub-distributions of $Y$ and $Z$ given the conditions (1), (2), and (3) by $F_{Y q}(y \mid x, \boldsymbol{\theta})$ and $F_{Z q}(z \mid x, \boldsymbol{\theta})$ where $q=1,2,3$, respectively.

Step 1. Compute (4.13) with (4.20) given an initial locally optimal design $\xi_{\boldsymbol{\theta}_{0}}=$ 
$\left\{x_{i}, w_{i}\right\}_{1}^{K}$ and the true parameters $\boldsymbol{\theta}$ :

$$
\begin{array}{ll}
p_{i 1}=\frac{\beta_{1}}{\exp \left(-\left(\theta_{0}+\theta_{3} x_{i}\right)\right)} & \text { for } 0<y<z<\infty, \\
p_{i 2}=\frac{\beta_{2}}{\exp \left(-\left(\theta_{0}+\theta_{3} x_{i}\right)\right)} & \text { for } 0<z<y<\infty, \\
p_{i 3}=\frac{\exp \left(-\left(\theta_{0}+\theta_{3} x_{i}\right)\right)-\beta_{1}-\beta_{2}}{\exp \left(-\left(\theta_{0}+\theta_{3} x_{i}\right)\right)} & \text { for } 0<y=z<\infty .
\end{array}
$$

Step 2. Set an initial sample size $n_{0}$, then compute $n_{0} w_{i} p_{i q}=n_{0} \times w_{i} \times p_{i q}$ where $q=1,2,3$, and $i=1, \ldots, K$ (see Chapter 2.1).

Step 3. Generate $n_{0} w_{i} p_{i q}$ random samples from $u 1_{i q} \sim \operatorname{Uniform}\left[0, p_{i q}\right], i=1, \ldots, K$.

Step 4. Generate $n_{0} w_{i} p_{i q}$ random samples from $y_{i q} \sim F_{Y q}^{-1}\left(u 1_{i q} \mid x_{i}, \boldsymbol{\theta}\right), i=1, \ldots, K$.

Step 5. Generate $n_{0} w_{i} p_{i q}$ random samples from $u 2_{i q} \sim \operatorname{Uniform}[0,1], i=1, \ldots, K$.

Step 6. Generate $n_{0} w_{i} p_{i q}$ random samples from $z_{i q} \sim F_{Z q \mid y_{i q}}^{-1}\left(u 2_{i q} \mid y_{i q}, x_{i}, \boldsymbol{\theta}\right), i=$ $1, \ldots, K$.

Step 7. Select predetermined cutoff values $v_{1}^{*}$ and $v_{2}^{*}$. 
Step 8. Dichotomizing as in equation (4.11), compute empirical outcome probabilities: $\widehat{p}_{11}\left(x_{i}\right), \widehat{p}_{10}\left(x_{i}\right), \widehat{p}_{01}\left(x_{i}\right)$, and $\widehat{p}_{00}\left(x_{i}\right)$.

Step 9. Find the maximum likelihood estimates of the true parameter $\boldsymbol{\theta}$ from the log-likelihood function (4.18). Let $\widehat{\boldsymbol{\theta}}$ denote this estimate of $\boldsymbol{\theta}$.

Step 10. By exchange algorithm (see Appendix A.5), find the locally optimal design $\xi_{\widehat{\boldsymbol{\theta}}}$ assuming the parameter vector is $\widehat{\boldsymbol{\theta}}$.

Step 11. Repeat steps 1-8 with $\xi_{\widehat{\boldsymbol{\theta}}}$ in place of $\xi_{\boldsymbol{\theta}_{0}}$ and $n_{1}=n-n_{0}$ in place of $n_{0}$.

Step 12. Estimate the parameters with all observations $n=n_{0}+n_{1}$.

Table A.1 and Figure A.1 demonstrate that this simulation does, indeed, sample from the bivariate Weibull distribution.

\begin{tabular}{|l|c|c|c||c|c|c|c||c|c|c|c|}
\hline \multicolumn{3}{|c||}{$x=.2$} & \multicolumn{4}{c||}{$x=.5$} & \multicolumn{4}{c|}{$x=.9$} \\
\hline$p_{11}$ & .043503 & $\widehat{p}_{11}$ & .043343 & $p_{11}$ & .181770 & $\widehat{p}_{11}$ & .181586 & $p_{11}$ & .472600 & $\widehat{p}_{11}$ & .472724 \\
\hline$p_{10}$ & .010457 & $\widehat{p}_{10}$ & .010401 & $p_{10}$ & .041414 & $\widehat{p}_{10}$ & .041299 & $p_{10}$ & .071254 & $\widehat{p}_{10}$ & .071418 \\
\hline$p_{01}$ & .051428 & $\widehat{p}_{01}$ & .051426 & $p_{01}$ & .102482 & $\widehat{p}_{01}$ & .103033 & $p_{01}$ & .111977 & $\widehat{p}_{01}$ & .111692 \\
\hline$p_{00}$ & .894612 & $\widehat{p}_{00}$ & .894830 & $p_{00}$ & .674334 & $\widehat{p}_{00}$ & .674082 & $p_{00}$ & .344168 & $\widehat{p}_{00}$ & .344166 \\
\hline
\end{tabular}

Table A.1: True outcome probabilities and simulated outcome probabilities $(n=$ $1,000,000)$ 


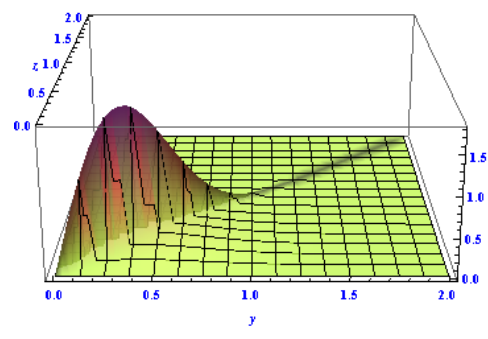

(a) True Density $(x=.2)$

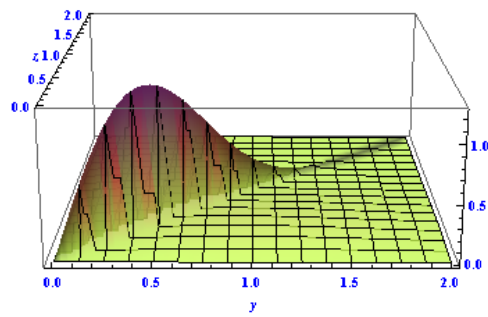

(c) True Density $(x=.5)$

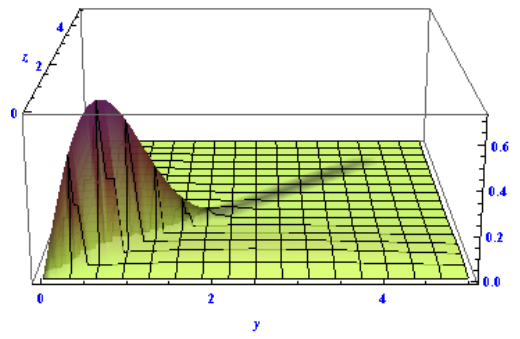

(e) True Density $(x=.9)$

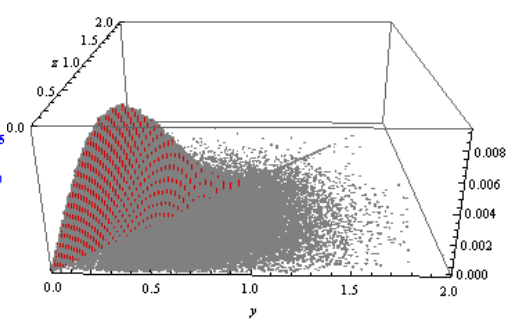

(b) Probability Histogram $(x=.2)$

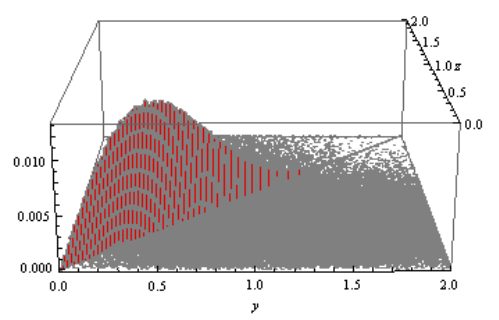

(d) Probability Histogram $(x=.5)$

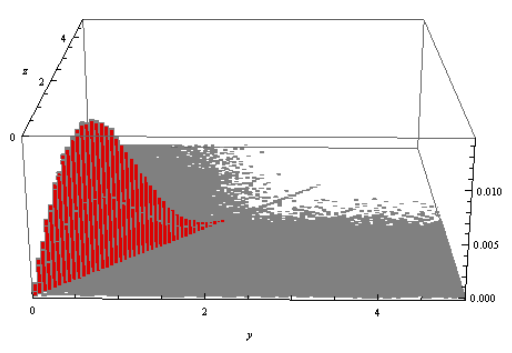

(f) Probability Histogram $(x=.9)$

Figure A.1: Comparisons of bivariate Weibull regression models with the probability histograms of the simulated observations 


\section{A.7 Locally Optimal Designs with Various Param- eter Values}

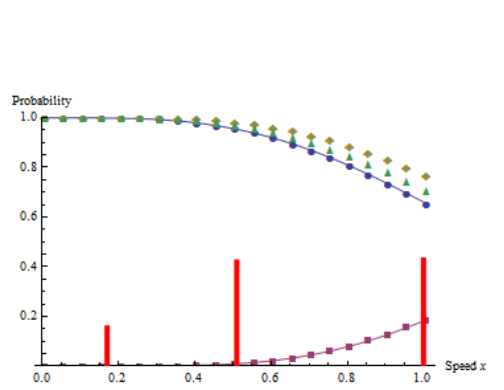

(a) $\theta_{0}=-3$

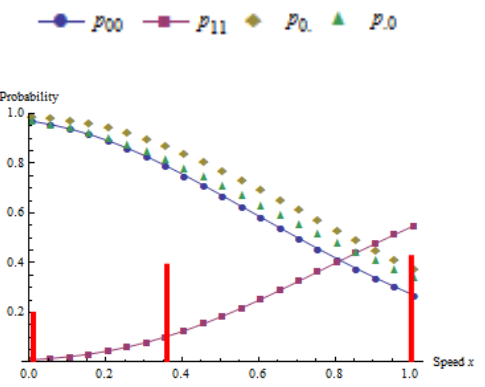

(b) $\theta_{0}=-2$

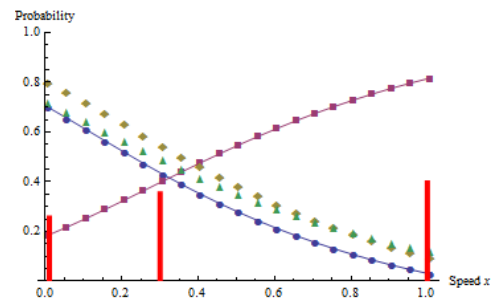

(c) $\theta_{0}=-1$

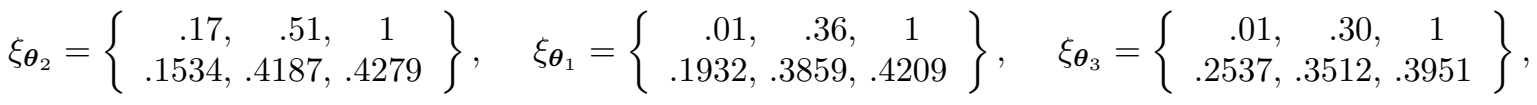

$$
\begin{aligned}
& \left|M\left(\xi_{\boldsymbol{\theta}_{2}}, \boldsymbol{\theta}_{2}\right)\right|^{1 / m}=.0076, \quad\left|M\left(\xi_{\boldsymbol{\theta}_{1}}, \boldsymbol{\theta}_{1}\right)\right|^{1 / m}=.0157, \quad\left|M\left(\xi_{\boldsymbol{\theta}_{3}}, \boldsymbol{\theta}_{3}\right)\right|^{1 / m}=.0194
\end{aligned}
$$

Figure A.2: D-optimal designs varying $\theta_{0}$

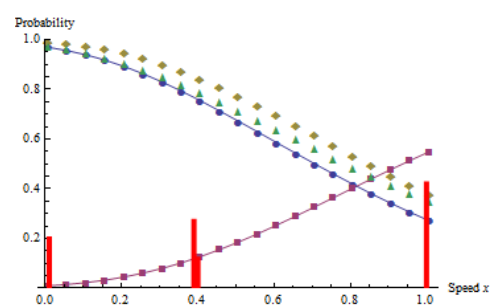

(a) $\theta_{1}=3$

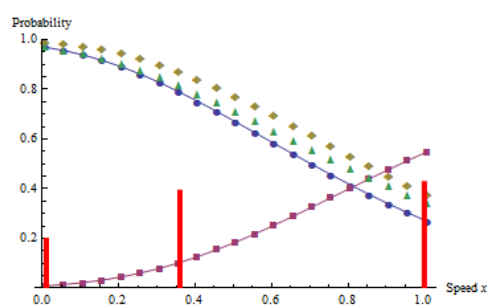

(b) $\theta_{1}=4$

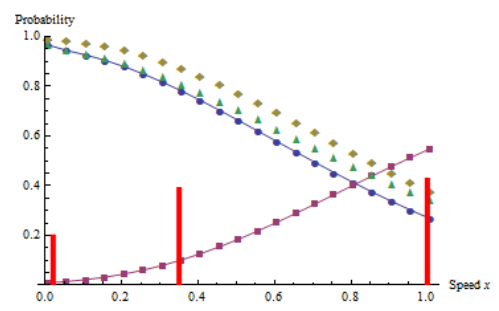

(c) $\theta_{1}=100$

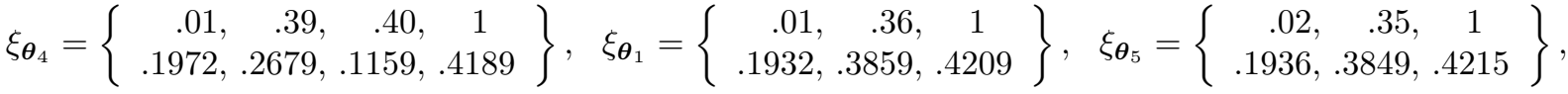

$$
\begin{aligned}
& \left|M\left(\xi_{\boldsymbol{\theta}_{4}}, \boldsymbol{\theta}_{4}\right)\right|^{1 / m}=.0153, \quad\left|M\left(\xi_{\boldsymbol{\theta}_{1}}, \boldsymbol{\theta}_{1}\right)\right|^{1 / m}=.0157, \quad\left|M\left(\xi_{\boldsymbol{\theta}_{5}}, \boldsymbol{\theta}_{5}\right)\right|^{1 / m}=.0064
\end{aligned}
$$

Figure A.3: D-optimal designs varying $\theta_{1}$ 


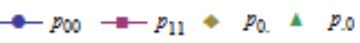

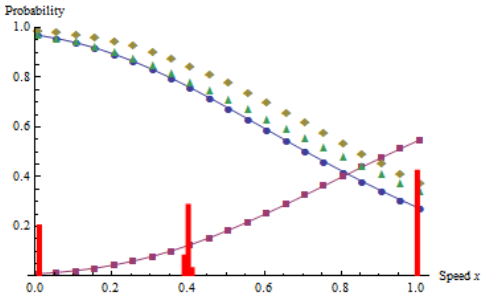

(a) $\theta_{2}=4$

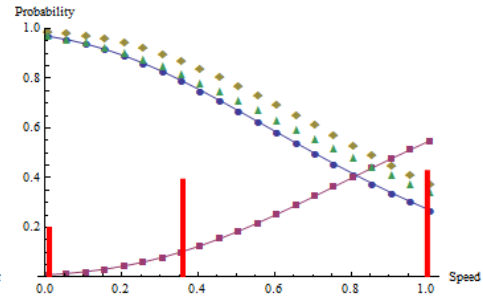

(b) $\theta_{2}=5$

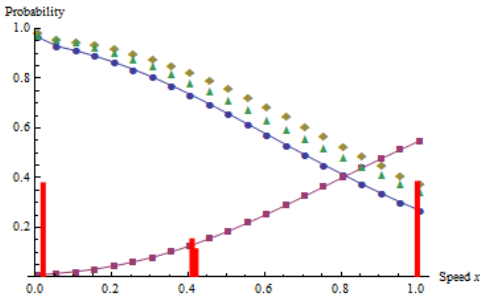

(c) $\theta_{2}=100$

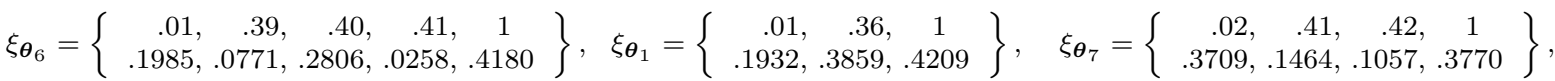

$\left|M\left(\xi_{\boldsymbol{\theta}_{6}}, \boldsymbol{\theta}_{6}\right)\right|^{1 / m}=.0162, \quad\left|M\left(\xi_{\boldsymbol{\theta}_{1}}, \boldsymbol{\theta}_{1}\right)\right|^{1 / m}=.0157, \quad\left|M\left(\xi_{\boldsymbol{\theta}_{7}}, \boldsymbol{\theta}_{7}\right)\right|^{1 / m}=.0067$

Figure A.4: D-optimal designs varying $\theta_{2}$

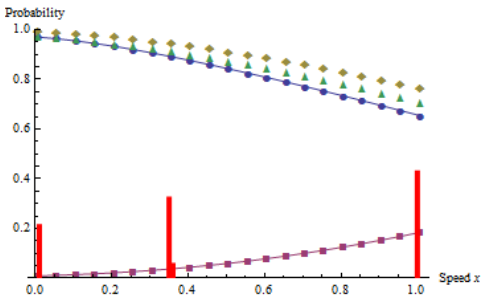

(a) $\theta_{3}=1$

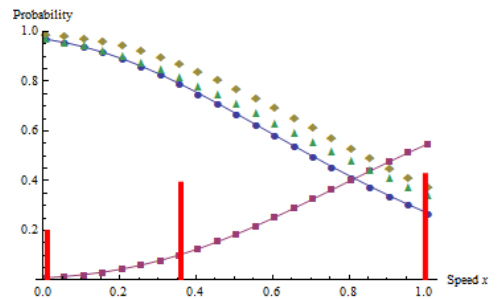

(b) $\theta_{3}=2$

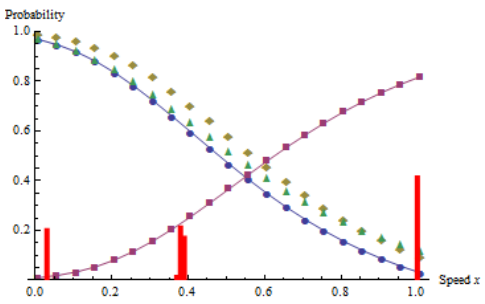

(c) $\theta_{3}=3$

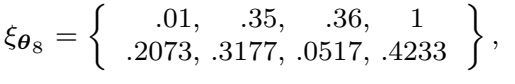

$$
\begin{aligned}
& \xi_{\boldsymbol{\theta}_{1}}=\left\{\begin{array}{ccc}
.01, & .36, & 1 \\
.1932, & .3859, & .4209
\end{array}\right\}
\end{aligned}
$$

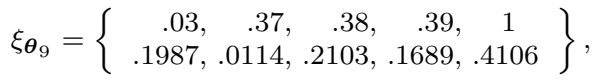

$$
\begin{aligned}
& \left|M\left(\xi_{\boldsymbol{\theta}_{8}}, \boldsymbol{\theta}_{8}\right)\right|^{1 / m}=.0122, \\
& \left|M\left(\xi_{\boldsymbol{\theta}_{1}}, \boldsymbol{\theta}_{1}\right)\right|^{1 / m}=.0157, \\
& \left|M\left(\xi_{\boldsymbol{\theta}_{9}}, \boldsymbol{\theta}_{9}\right)\right|^{1 / m}=.0214
\end{aligned}
$$

Figure A.5: D-optimal designs varying $\theta_{3}$ 


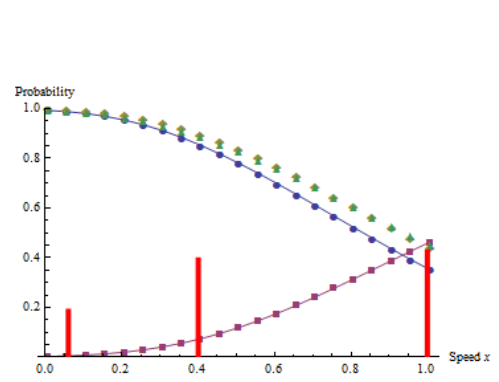

(a) $\tau=1$

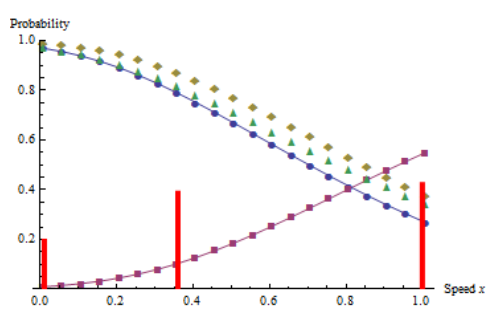

(b) $\tau=2$

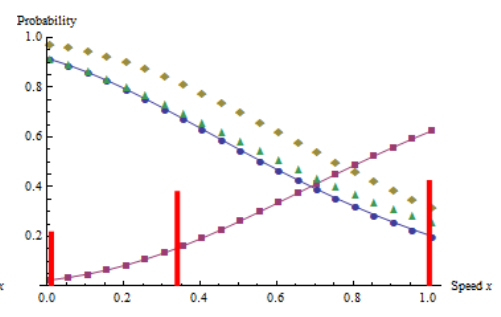

(c) $\tau=3$

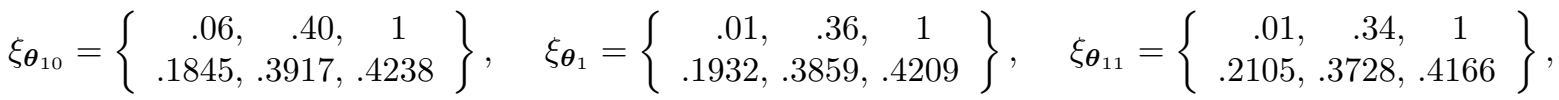

$$
\begin{aligned}
& \left|M\left(\xi_{\boldsymbol{\theta}_{10}}, \boldsymbol{\theta}_{10}\right)\right|^{1 / m}=.0205, \quad\left|M\left(\xi_{\boldsymbol{\theta}_{1}}, \boldsymbol{\theta}_{1}\right)\right|^{1 / m}=.0157, \quad\left|M\left(\xi_{\boldsymbol{\theta}_{11}}, \boldsymbol{\theta}_{11}\right)\right|^{1 / m}=.0118
\end{aligned}
$$

Figure A.6: D-optimal designs varying $\tau$

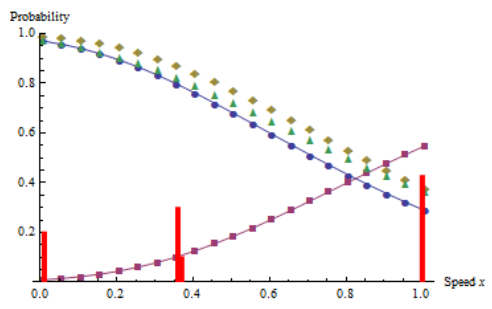

(a) $\beta_{1}=.05$

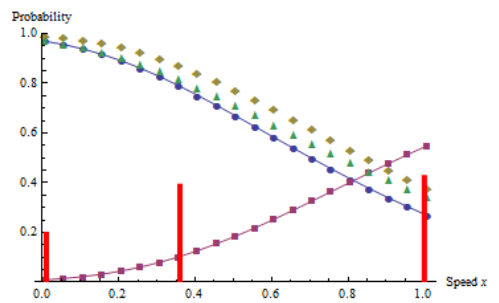

(b) $\beta_{1}=.1$

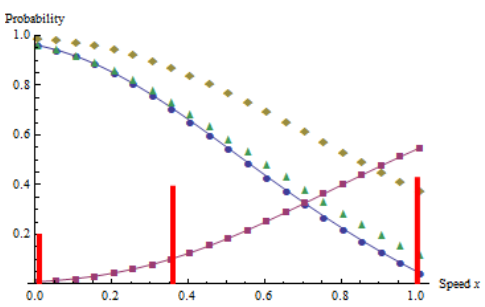

(c) $\beta_{1}=.7$

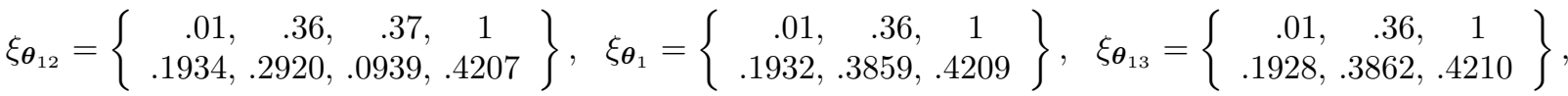

$$
\begin{aligned}
& \left|M\left(\xi_{\boldsymbol{\theta}_{12}}, \boldsymbol{\theta}_{12}\right)\right|^{1 / m}=.0132, \quad\left|M\left(\xi_{\boldsymbol{\theta}_{1}}, \boldsymbol{\theta}_{1}\right)\right|^{1 / m}=.0157, \quad\left|M\left(\xi_{\boldsymbol{\theta}_{13}}, \boldsymbol{\theta}_{13}\right)\right|^{1 / m}=.0280
\end{aligned}
$$

Figure A.7: D-optimal designs varying $\beta_{1}$ 


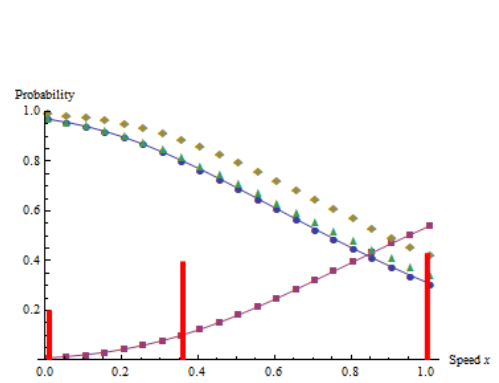

(a) $\beta_{2}=.1$

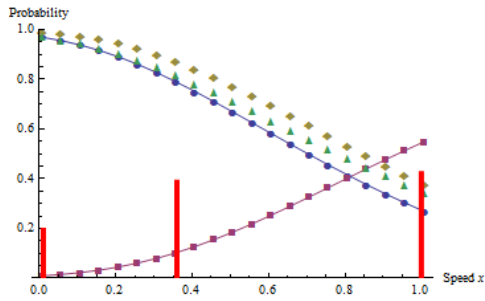

(b) $\beta_{2}=.2$

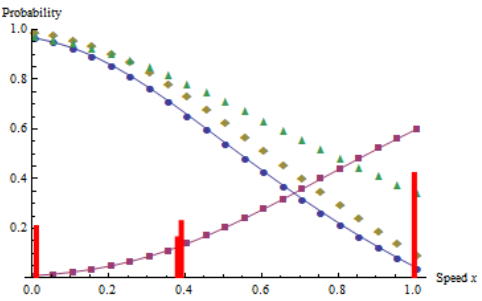

(c) $\beta_{2}=.8$

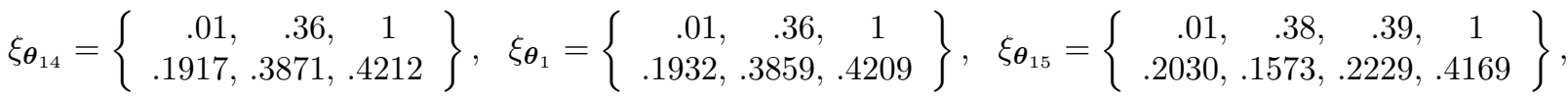

$$
\begin{aligned}
& \left|M\left(\xi_{\boldsymbol{\theta}_{14}}, \boldsymbol{\theta}_{14}\right)\right|^{1 / m}=.0150, \quad\left|M\left(\xi_{\boldsymbol{\theta}_{1}}, \boldsymbol{\theta}_{1}\right)\right|^{1 / m}=.0157, \quad\left|M\left(\xi_{\boldsymbol{\theta}_{15}}, \boldsymbol{\theta}_{15}\right)\right|^{1 / m}=.0240
\end{aligned}
$$

Figure A.8: D-optimal designs varying $\beta_{2}$ 


\section{A.8 A Trivariate Weibull Probability Density}

$f\left(y_{1}, y_{2}, y_{3}\right)$

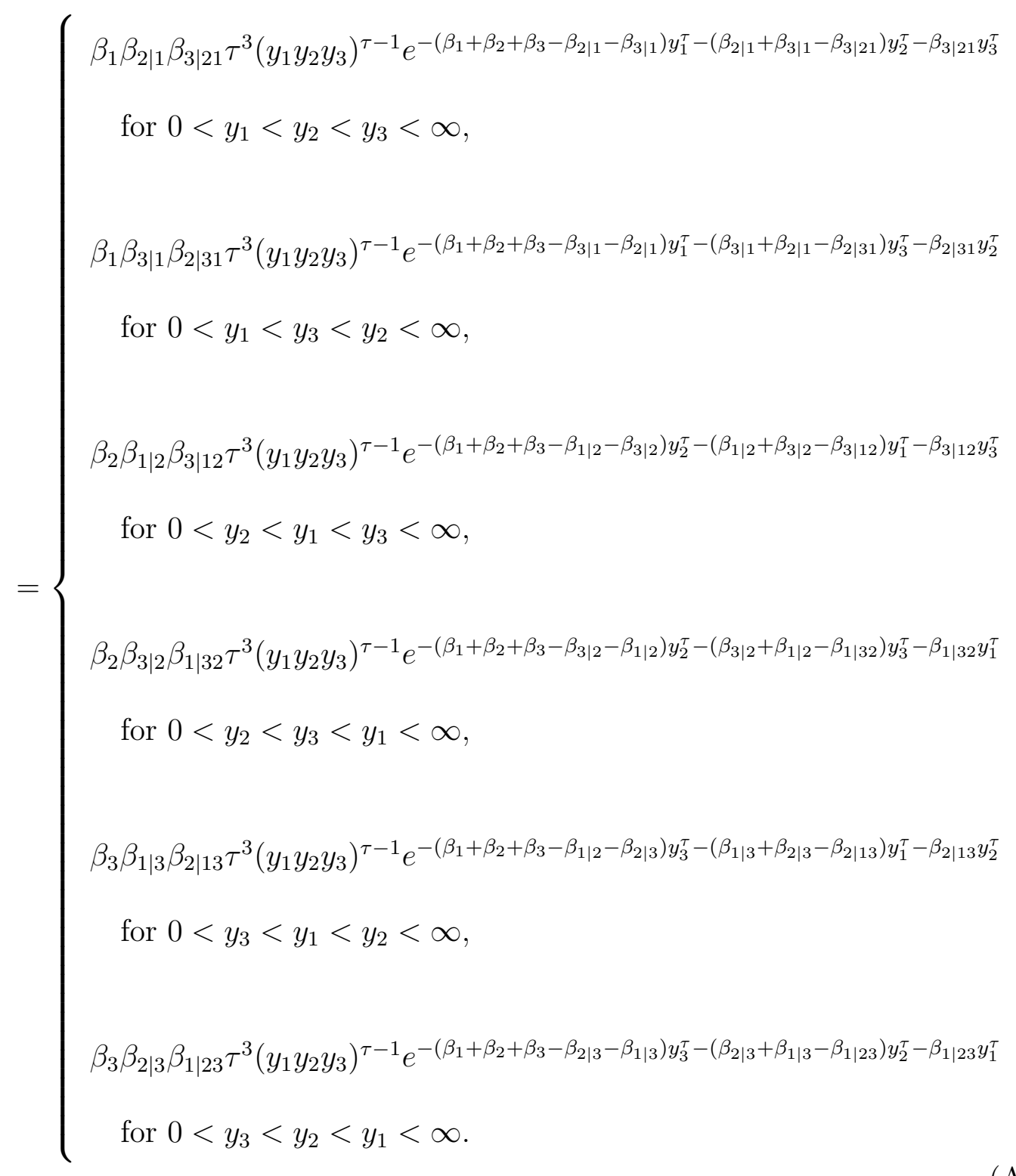




\section{A.9 Outcome Probabilities (Equation (5.7))}

$$
\begin{aligned}
p_{000} & =\frac{\beta_{3} \beta_{2 \mid 3}}{\left(\beta_{3}-\beta_{2 \mid 3}\right)\left(\beta_{2 \mid 3}-\beta_{1 \mid 23}\right)}\left(1-e^{-\beta_{1 \mid 23} v^{* \tau}}\right)-\frac{\beta_{3} \beta_{1 \mid 23}}{\left(\beta_{3}-\beta_{2 \mid 3}\right)\left(\beta_{2 \mid 3}-\beta_{1 \mid 23}\right)}\left(1-e^{-\beta_{2 \mid 3} v^{* \tau}}\right) \\
& -\frac{\beta_{3} \beta_{2 \mid 3}}{\left(\beta_{3}-\beta_{2 \mid 3}\right)\left(\beta_{3}-\beta_{1 \mid 23}\right)}\left(1-e^{-\beta_{1 \mid 23} v^{* \tau}}\right)+\frac{\beta_{2 \mid 3} \beta_{1 \mid 23}}{\left(\beta_{3}-\beta_{2 \mid 3}\right)\left(\beta_{3}-\beta_{1 \mid 23}\right)}\left(1-e^{-\beta_{3} v^{* \tau}}\right) ; \\
p_{100} & =\frac{\beta_{3} \beta_{2 \mid 3}}{\left(\beta_{3}-\beta_{2 \mid 3}\right)} e^{-\beta_{1 \mid 23} v^{* \tau}} \\
& \times\left(\frac{1}{\left(\beta_{2 \mid 3}-\beta_{1 \mid 23}\right)}\left(1-e^{\left.-\left(\beta_{2 \mid 3}-\beta_{1 \mid 23}\right) v^{* \tau}\right)}-\frac{1}{\left(\beta_{3}-\beta_{1 \mid 23}\right)}\left(1-e^{-\left(\beta_{3}-\beta_{1 \mid 23}\right) v^{* \tau}}\right)\right) ;\right. \\
p_{110} & =\frac{\beta_{3}}{\left(\beta_{3}-\beta_{2 \mid 3}\right)} e^{-\beta_{2 \mid 3} v^{* \tau}}\left(1-e^{-\left(\beta_{3}-\beta_{2 \mid 3}\right) v^{* \tau}}\right) ; \\
p_{111} & =e^{-\beta_{3} v^{* \tau}} \cdot
\end{aligned}
$$




\section{A.10 Outcome Probabilities with Regression Terms (Equation (5.8))}

$$
\begin{aligned}
& p_{000}=\frac{e^{-\eta_{1}\left(\boldsymbol{x} ; \boldsymbol{\vartheta}_{1}\right)} e^{-\eta_{2}\left(\boldsymbol{x} ; \boldsymbol{\vartheta}_{2}\right)}}{\left(e^{-\eta_{1}\left(\boldsymbol{x} ; \boldsymbol{\vartheta}_{1}\right)}-e^{-\eta_{2}\left(\boldsymbol{x} ; \boldsymbol{\vartheta}_{2}\right)}\right)\left(e^{-\eta_{2}\left(\boldsymbol{x} ; \boldsymbol{\vartheta}_{2}\right)}-e^{-\eta_{3}\left(\boldsymbol{x} ; \boldsymbol{\vartheta}_{3}\right)}\right)}\left(1-e^{-e^{-\eta_{3}\left(\boldsymbol{x} ; \boldsymbol{\vartheta}_{3}\right)} v^{* \tau}}\right) \\
& -\frac{e^{-\eta_{1}\left(\boldsymbol{x} ; \boldsymbol{\vartheta}_{1}\right)} e^{-\eta_{3}\left(\boldsymbol{x} ; \boldsymbol{\vartheta}_{3}\right)}}{\left(e^{-\eta_{1}\left(\boldsymbol{x} ; \boldsymbol{\vartheta}_{1}\right)}-e^{-\eta_{2}\left(\boldsymbol{x} ; \boldsymbol{\vartheta}_{2}\right)}\right)\left(e^{-\eta_{2}\left(\boldsymbol{x} ; \boldsymbol{\vartheta}_{2}\right)}-e^{-\eta_{3}\left(\boldsymbol{x} ; \boldsymbol{\vartheta}_{3}\right)}\right)}\left(1-e^{-e^{-\eta_{2}\left(\boldsymbol{x} ; \boldsymbol{\vartheta}_{2}\right)} v^{* \tau}}\right) \\
& -\frac{e^{-\eta_{1}\left(\boldsymbol{x} ; \boldsymbol{\vartheta}_{1}\right)} e^{-\eta_{2}\left(\boldsymbol{x} ; \boldsymbol{\vartheta}_{2}\right)}}{\left(e^{-\eta_{1}\left(\boldsymbol{x} ; \boldsymbol{\vartheta}_{1}\right)}-e^{-\eta_{2}\left(\boldsymbol{x} ; \boldsymbol{\vartheta}_{2}\right)}\right)\left(e^{-\eta_{1}\left(\boldsymbol{x} ; \boldsymbol{\vartheta}_{1}\right)}-e^{-\eta_{3}\left(\boldsymbol{x} ; \boldsymbol{\vartheta}_{3}\right)}\right)}\left(1-e^{-e^{-\eta_{3}\left(\boldsymbol{x} ; \boldsymbol{\vartheta}_{3}\right)} v^{* \tau}}\right) \\
& +\frac{e^{-\eta_{2}\left(\boldsymbol{x} ; \boldsymbol{\vartheta}_{2}\right)} e^{-\eta_{3}\left(\boldsymbol{x} ; \boldsymbol{\vartheta}_{3}\right)}}{\left(e^{-\eta_{1}\left(\boldsymbol{x} ; \boldsymbol{\vartheta}_{1}\right)}-e^{-\eta_{2}\left(\boldsymbol{x} ; \boldsymbol{\vartheta}_{2}\right)}\right)\left(e^{-\eta_{1}\left(\boldsymbol{x} ; \boldsymbol{\vartheta}_{1}\right)}-e^{-\eta_{3}\left(\boldsymbol{x} ; \boldsymbol{\vartheta}_{3}\right)}\right)}\left(1-e^{-e^{-\eta_{1}\left(\boldsymbol{x} ; \boldsymbol{\vartheta}_{1}\right)} v^{* \tau}}\right) \\
& p_{100}=\frac{e^{-\eta_{1}\left(\boldsymbol{x} ; \boldsymbol{\vartheta}_{1}\right)} e^{-\eta_{2}\left(\boldsymbol{x} ; \boldsymbol{\vartheta}_{2}\right)}}{\left(e^{-\eta_{1}\left(\boldsymbol{x} ; \boldsymbol{\vartheta}_{1}\right)}-e^{-\eta_{2}\left(\boldsymbol{x} ; \boldsymbol{\vartheta}_{2}\right)}\right)} e^{-e^{-\eta_{3}\left(\boldsymbol{x} ; \boldsymbol{\vartheta}_{3}\right)} v^{* \tau}} \\
& \times\left(\frac{1}{\left(e^{-\eta_{2}\left(\boldsymbol{x} ; \boldsymbol{\vartheta}_{2}\right)}-e^{-\eta_{3}\left(\boldsymbol{x} ; \boldsymbol{\vartheta}_{3}\right)}\right)}\left(1-e^{-\left(e^{-\eta_{2}\left(\boldsymbol{x} ; \boldsymbol{\vartheta}_{2}\right)}-e^{-\eta_{3}\left(\boldsymbol{x} ; \boldsymbol{\vartheta}_{3}\right)}\right) v^{* \tau}}\right)\right) \\
& -\frac{e^{-\eta_{1}\left(\boldsymbol{x} ; \boldsymbol{\vartheta}_{1}\right)} e^{-\eta_{2}\left(\boldsymbol{x} ; \boldsymbol{\vartheta}_{2}\right)}}{\left(e^{-\eta_{1}\left(\boldsymbol{x} ; \boldsymbol{\vartheta}_{1}\right)}-e^{-\eta_{2}\left(\boldsymbol{x} ; \boldsymbol{\vartheta}_{2}\right)}\right)} e^{-e^{-\eta_{3}\left(\boldsymbol{x} ; \boldsymbol{\vartheta}_{3}\right)} v^{* \tau}} \\
& \times\left(\frac{1}{\left(e^{-\eta_{1}\left(\boldsymbol{x} ; \boldsymbol{\vartheta}_{1}\right)}-e^{-\eta_{3}\left(\boldsymbol{x} ; \boldsymbol{\vartheta}_{3}\right)}\right)}\left(1-e^{-\left(e^{-\eta_{1}\left(\boldsymbol{x} ; \boldsymbol{\vartheta}_{1}\right)}-e^{-\eta_{3}\left(\boldsymbol{x} ; \boldsymbol{\vartheta}_{3}\right)}\right) v^{* \tau}}\right)\right) \\
& p_{110}=\frac{e^{-\eta_{1}\left(\boldsymbol{x} ; \boldsymbol{\vartheta}_{1}\right)}}{\left(e^{-\eta_{1}\left(\boldsymbol{x} ; \boldsymbol{\vartheta}_{1}\right)}-e^{-\eta_{2}\left(\boldsymbol{x} ; \boldsymbol{\vartheta}_{2}\right)}\right)} e^{-e^{-\eta_{2}\left(\boldsymbol{x} ; \boldsymbol{\vartheta}_{2}\right)} v^{* \tau}}\left(1-e^{-\left(e^{-\eta_{1}\left(\boldsymbol{x} ; \boldsymbol{\vartheta}_{1}\right)}-e^{-\eta_{2}\left(\boldsymbol{x} ; \boldsymbol{\vartheta}_{2}\right)}\right) v^{* \tau}}\right) ; \\
& p_{111}=e^{-e^{-\eta_{1}\left(\boldsymbol{x} ; \boldsymbol{\vartheta}_{1}\right)} v^{* \tau}} .
\end{aligned}
$$




\section{A.11 Outcome Probabilities with Linear Regres- sion Terms (Equation (5.12))}

$$
\begin{aligned}
& p_{000}=\frac{e^{-\left(\theta_{0}+\theta_{1} x\right)} e^{-\left(\theta_{0}+\theta_{2} x\right)}}{\left(e^{-\left(\theta_{0}+\theta_{1} x\right)}-e^{-\left(\theta_{0}+\theta_{2} x\right)}\right)\left(e^{-\left(\theta_{0}+\theta_{2} x\right)}-e^{-\left(\theta_{0}+\theta_{3} x\right)}\right)}\left(1-e^{-e^{-\left(\theta_{0}+\theta_{3} x\right)} v^{* \tau}}\right) \\
& -\frac{e^{-\left(\theta_{0}+\theta_{1} x\right)} e^{-\left(\theta_{0}+\theta_{3} x\right)}}{\left(e^{-\left(\theta_{0}+\theta_{1} x\right)}-e^{-\left(\theta_{0}+\theta_{2} x\right)}\right)\left(e^{-\left(\theta_{0}+\theta_{2} x\right)}-e^{-\left(\theta_{0}+\theta_{3} x\right)}\right)}\left(1-e^{-e^{-\left(\theta_{0}+\theta_{2} x\right)} v^{* \tau}}\right) \\
& -\frac{e^{-\left(\theta_{0}+\theta_{1} x\right)} e^{-\left(\theta_{0}+\theta_{2} x\right)}}{\left(e^{-\left(\theta_{0}+\theta_{1} x\right)}-e^{-\left(\theta_{0}+\theta_{2} x\right)}\right)\left(e^{-\left(\theta_{0}+\theta_{1} x\right)}-e^{-\left(\theta_{0}+\theta_{3} x\right)}\right)}\left(1-e^{-e^{-\left(\theta_{0}+\theta_{3} x\right)} v^{* \tau}}\right) \\
& +\frac{e^{-\left(\theta_{0}+\theta_{2} x\right)} e^{-\left(\theta_{0}+\theta_{3} x\right)}}{\left(e^{-\left(\theta_{0}+\theta_{1} x\right)}-e^{-\left(\theta_{0}+\theta_{2} x\right)}\right)\left(e^{-\left(\theta_{0}+\theta_{1} x\right)}-e^{-\left(\theta_{0}+\theta_{3} x\right)}\right)}\left(1-e^{-e^{-\left(\theta_{0}+\theta_{1} x\right)} v^{* \tau}}\right) ; \\
& p_{100}=\frac{e^{-\left(\theta_{0}+\theta_{1} x\right)} e^{-\left(\theta_{0}+\theta_{2} x\right)}}{\left(e^{-\left(\theta_{0}+\theta_{1} x\right)}-e^{-\left(\theta_{0}+\theta_{2} x\right)}\right)} e^{-e^{-\left(\theta_{0}+\theta_{3} x\right)} v^{* \tau}} \\
& \times\left(\frac{1}{\left(e^{-\left(\theta_{0}+\theta_{2} x\right)}-e^{-\left(\theta_{0}+\theta_{3} x\right)}\right)}\left(1-e^{-\left(e^{-\left(\theta_{0}+\theta_{2} x\right)}-e^{-\left(\theta_{0}+\theta_{3} x\right)}\right) v^{* \tau}}\right)\right) \\
& -\frac{e^{-\left(\theta_{0}+\theta_{1} x\right)} e^{-\left(\theta_{0}+\theta_{2} x\right)}}{\left(e^{-\left(\theta_{0}+\theta_{1} x\right)}-e^{-\left(\theta_{0}+\theta_{2} x\right)}\right)} e^{-e^{-\left(\theta_{0}+\theta_{3} x\right)} v^{* \tau}} \\
& \times\left(\frac{1}{\left(e^{-\left(\theta_{0}+\theta_{1} x\right)}-e^{-\left(\theta_{0}+\theta_{3} x\right)}\right)}\left(1-e^{-\left(e^{-\left(\theta_{0}+\theta_{1} x\right)}-e^{-\left(\theta_{0}+\theta_{3} x\right)}\right) v^{* \tau}}\right)\right) ; \\
& p_{110}=\frac{e^{-\left(\theta_{0}+\theta_{1} x\right)}}{\left(e^{-\left(\theta_{0}+\theta_{1} x\right)}-e^{-\left(\theta_{0}+\theta_{2} x\right)}\right)} e^{-e^{-\left(\theta_{0}+\theta_{2} x\right)} v^{* \tau}}\left(1-e^{-\left(e^{-\left(\theta_{0}+\theta_{1} x\right)}-e^{-\left(\theta_{0}+\theta_{2} x\right)}\right) v^{* \tau}}\right) ; \\
& p_{111}=e^{-e^{-\left(\theta_{0}+\theta_{1} x\right)} v^{* \tau}} .
\end{aligned}
$$




\section{A.12 A Proof of the Equation (5.18)}

Let $M(\xi)$ be the $(m \times m)$ information matrix of the design $\xi$ where $m$ is the number

of parameters; let $\bar{\xi}$ be a design with unit weight at the point $x$, and let the weighted average design be

$$
\xi^{\prime}=(1-\alpha) \xi+\alpha \bar{\xi}, 0 \leq \alpha \leq 1
$$

Then the information matrix from $\xi^{\prime}$ is

$$
M\left(\xi^{\prime}\right)=(1-\alpha) M(\xi)+\alpha M(\bar{\xi})
$$

Assume that $\Psi\{M(\xi)\}$ is a convex function:

$$
\Psi\{(1-\alpha) M(\xi)+\alpha M(\bar{\xi})\} \leq(1-\alpha) \Psi\{M(\xi)\}+\alpha \Psi\{M(\bar{\xi})\}
$$

Consider the directional derivative of $\Psi\{M(\xi)\}$ in the direction of $\bar{\xi}$ :

$$
\lim _{\alpha \rightarrow 0+} \frac{[\Psi\{(1-\alpha) M(\xi)+\alpha M(\bar{\xi})\}-\Psi\{M(\xi)\}]}{\alpha} .
$$

Since the equation (A.11) is a singular, 


$$
\begin{aligned}
& \lim _{\alpha \rightarrow 0+} \frac{[\Psi\{(1-\alpha) M(\xi)+\alpha M(\bar{\xi})\}-\Psi\{M(\xi)\}]}{\alpha} \\
& =\operatorname{tr}\left(\lim _{\alpha \rightarrow 0+} \frac{[\Psi\{(1-\alpha) M(\xi)+\alpha M(\bar{\xi})\}-\Psi\{M(\xi)\}]}{\alpha}\right) \\
& =\operatorname{tr}\left(\lim _{\alpha \rightarrow 0+} \frac{[\Psi\{M(\xi)+\alpha(M(\bar{\xi})-M(\xi))\}-\Psi\{M(\xi)\}]}{\alpha}\right) \\
& =\operatorname{tr}\left(\lim _{\alpha \rightarrow 0+} \frac{[\Psi\{M(\xi)+\alpha(M(\bar{\xi})-M(\xi))\}-\Psi\{M(\xi)\}]}{\alpha(M(\bar{\xi})-M(\xi))}(M(\bar{\xi})-M(\xi))\right) \\
& =\operatorname{tr}\left(\frac{\partial \Psi\{M(\xi)\}}{\partial M(\xi)}(M(\bar{\xi})-M(\xi))\right) \\
& =\operatorname{tr}\left(\frac{\partial \Psi\{M(\xi)\}}{\partial M(\xi)} M(\bar{\xi})\right)-\operatorname{tr}\left(\frac{\partial \Psi\{M(\xi)\}}{\partial M(\xi)} M(\xi)\right) .
\end{aligned}
$$

If we assume

$$
\Psi\{M(\xi)\}=\operatorname{tr}\left(A(M(\xi))^{-1}\right),
$$

where $A$ is a $(q \times m)$ matrix, $q$ is the number of linear combinations of parameters to be estimated, then

$$
\frac{\partial \Psi\{M(\xi)\}}{\partial M(\xi)}=-(M(\xi))^{-1} A(M(\xi))^{-1} .
$$

Inserting (A.14) into (A.12), then (A.12) becomes

$$
\operatorname{tr}\left(-(M(\xi))^{-1} A(M(\xi))^{-1} M(\bar{\xi})\right)+\operatorname{tr}\left((M(\xi))^{-1} A\right) .
$$

From the convexity of $M(\xi)$, (A.15) must be positive at any $M(\xi)$, and it has a minimum when equation (A.15) equals 0 :

$$
\operatorname{tr}\left((M(\xi))^{-1} A(M(\xi))^{-1} M(\bar{\xi})\right) \leq \operatorname{tr}\left((M(\xi))^{-1} A\right) .
$$


If $A$ is of rank $s<q$, then $A$ can be expressed in the form of $A=C C^{T}$, where $C$ is a $m \times s$ matrix of rank $s,($ A.16) becomes

$$
\operatorname{tr}\left(M(\bar{\xi})(M(\xi))^{-1} C C^{T}(M(\xi))^{-1}\right) \leq \operatorname{tr}\left(C^{T}(M(\xi))^{-1} C\right)
$$

Setting $s=1$, and replacing $M(\bar{\xi})$ to $\mu(x)$, then (A.17) becomes (5.18), it is the end of a proof of the equation (5.18). 


\section{A.13 The Exchange Algorithm for c-Optimality}

Step 1. Select an initial design $\xi_{s}=\left\{x_{i}, w_{i}\right\}_{1}^{k}$, where $w_{i}=n_{i} / n$ and $n=\sum_{1}^{k} n_{i}$.

Step 2. Compute

$d_{1}\left(x, \xi_{s}, \boldsymbol{\theta}\right)=\left(E S_{\alpha}^{\prime}\right)^{T} M^{-1}\left(\xi_{s}\right) E S_{\alpha}^{\prime}-\operatorname{tr}\left\{\mu(x) M^{-1}\left(\xi_{s}\right) E S_{\alpha}^{\prime}\left(E S_{\alpha}^{\prime}\right)^{T} M^{-1}\left(\xi_{s}\right)\right\}$

for all support points $x \in \mathfrak{X}$, and

$d_{2}\left(x, \xi_{s}, \boldsymbol{\theta}\right)=\left(E S_{\alpha}^{\prime}\right)^{T} M^{-1}\left(\xi_{s}\right) E S_{\alpha}^{\prime}-\operatorname{tr}\left\{\mu(x) M^{-1}\left(\xi_{s}\right) E S_{\alpha}^{\prime}\left(E S_{\alpha}^{\prime}\right)^{T} M^{-1}\left(\xi_{s}\right)\right\}$

for all support points $x \in \mathfrak{X}_{\mathfrak{s}}$ where $\mathfrak{X}_{\mathfrak{s}}$ is the set of support points in $\xi_{s}$.

Step 3. Then the next design point is

$$
x_{s+1}= \begin{cases}\arg \min _{x \in \mathfrak{X}} d_{1}\left(x, \xi_{s}, \boldsymbol{\theta}\right), & \text { if }-\max \left[d_{2}\left(x, \xi_{s}, \boldsymbol{\theta}\right)\right] \geq \min \left[d_{1}\left(x, \xi_{s}, \boldsymbol{\theta}\right)\right], \\ \arg \max _{x \in \mathfrak{X}_{\mathfrak{s}}} d_{2}\left(x, \xi_{s}, \boldsymbol{\theta}\right), & \text { otherwise. }\end{cases}
$$

Step 4. The new design $\xi_{s+1}$ is defined to be

$$
\xi_{s+1}=\left(1-\alpha_{s}\right) \xi_{s}+\alpha_{s} \xi\left(x_{s+1}\right)
$$

where $\xi\left(x_{s+1}\right)=\left\{x_{s+1}, 1\right\}$ is said to be a unit design at the design point $x_{s+1}$, 
and

$$
\alpha_{s}= \begin{cases}\frac{1}{s+1}, \quad \text { if }-\max \left[d_{2}\left(x, \xi_{s}, \boldsymbol{\theta}\right)\right] \geq \min \left[d_{1}\left(x, \xi_{s}, \boldsymbol{\theta}\right)\right], \\ -\min \left(\frac{1}{s+1}, \frac{w_{s+1}}{1-w_{s+1}}\right), & \text { otherwise. }\end{cases}
$$

where $w_{s+1}$ is the design weight of $x_{s+1}=\arg \max _{x \in \mathfrak{X}_{\mathfrak{s}}} d_{2}\left(x, \xi_{s}, \boldsymbol{\theta}\right)$ in the design $\xi_{s}$. Through the step 2 and 3, we add new good design points from $\mathfrak{X}$ to the design $\xi_{s}$ or give more weight on good design points in $\xi_{s}$. Otherwise we remove bad design points from $\xi_{s}$ or reduce weight on a bad design points in $\xi_{s}$.

Step 5. Compute

$$
\min \left|\left(E S_{\alpha}^{\prime}\right)^{T} M^{-1}\left(\xi_{s+1}\right) E S_{\alpha}^{\prime}-\operatorname{tr}\left\{\mu(x) M^{-1}\left(\xi_{s+1}\right) E S_{\alpha}^{\prime}\left(E S_{\alpha}^{\prime}\right)^{T} M^{-1}\left(\xi_{s+1}\right)\right\}\right|
$$

for all support points $x \in \mathfrak{X}$.

Step 6. Iterate steps 2-5 until (A.18) is smaller than $\epsilon$ which is an arbitrarily small positive number. Or find minimum of (A.18) within a predetermined number of iterations. 


\section{A.14 Simulation Study II}

Let the trivariate Weibull density (5.6) containing (5.12) be $g\left(y_{1}, y_{2}, y_{3} \mid x, \boldsymbol{\theta}\right)$. Define the marginal density function of $Y_{3}$ to be $g\left(y_{3} \mid x, \boldsymbol{\theta}\right)$, and its cumulative function to be $G\left(y_{3} \mid x, \boldsymbol{\theta}\right)$. Define the conditional density function of $Y_{2} \mid Y_{3}$ to be $g\left(y_{2} \mid y_{3}, x, \boldsymbol{\theta}\right)$, and its cumulative function to be $G\left(y_{2} \mid y_{3}, x, \boldsymbol{\theta}\right)$. Also define the conditional density function of $Y_{1} \mid Y_{2}, Y_{3}$ to be $g\left(y_{1} \mid y_{2}, y_{3}, x, \boldsymbol{\theta}\right)$, and its cumulative function to be $G\left(y_{1} \mid y_{2}, y_{3}, x, \boldsymbol{\theta}\right)$.

Step 1. Select an initial design $\xi_{F}=\left\{x_{i}, w_{i}\right\}_{1}^{K}$, and an initial sample size $n_{0}$. Then compute $n_{0} w_{i}=n_{0} \times w_{i}, i=1, \ldots, K$ (see Chapter 2.1).

Step 2. Generate $n_{0} w_{i}$ random samples from $u 1 \sim$ Uniform $[0,1], i=1, \ldots, K$.

Step 3. Generate $n_{0} w_{i}$ random samples from $y_{3 i} \sim G_{Y 3}^{-1}\left(u 1 \mid x_{i}, \boldsymbol{\theta}\right), i=1, \ldots, K$.

Step 4. Generate $n_{0} w_{i}$ random samples from $u 2 \sim$ Uniform $[0,1], i=1, \ldots, K$.

Step 5. Generate $n_{0} w_{i}$ random samples from $y_{2 i} \sim G_{Y 2 \mid Y 3}^{-1}\left(u 2 \mid y_{3 i}, x_{i}, \boldsymbol{\theta}\right), i=1, \ldots, K$.

Step 6. Generate $n_{0} w_{i}$ random samples from $u 3 \sim$ Uniform $[0,1], i=1, \ldots, K$. 
Step 7. Generate $n_{0} w_{i}$ random samples from $y_{1 i} \sim G_{Y 1 \mid Y 2, Y 3}^{-1}\left(u 3 \mid y_{2 i}, y_{3 i}, x_{i}, \boldsymbol{\theta}\right), i=$ $1, \ldots, K$.

Step 8. Select predetermined cutoff values $v^{*}$.

Step 9. Dichotomizing $\left\{y_{1 i}, y_{2 i}, y_{3 i}\right\}$ as in equation (6.3), compute empirical outcome probabilities: $\widehat{p}_{111}\left(x_{i}\right), \widehat{p}_{110}\left(x_{i}\right), \widehat{p}_{100}\left(x_{i}\right)$, and $\widehat{p}_{000}\left(x_{i}\right)$.

Step 10. Find the maximum likelihood estimates $\widehat{\boldsymbol{\theta}}$ of the true parameter $\boldsymbol{\theta}$ from the log-likelihood function (5.9).

Step 11. By using the exchange algorithm (see Appendix A.13), find the c-optimal design $\xi_{\widehat{\boldsymbol{\theta}}}$ assuming the parameter vector is $\widehat{\boldsymbol{\theta}}$.

Step 12. Compute the efficiency of the two-stage optimal design:

Efficiency $=\left(\frac{\left(E S_{\alpha}^{\prime}\right)^{T} M^{-1}\left(\xi_{\boldsymbol{\theta}}, \boldsymbol{\theta}\right) E S_{\alpha}^{\prime}}{p_{0}\left(\left(E S_{\alpha}^{\prime}\right)^{T} M^{-1}\left(\xi_{\boldsymbol{\theta}_{0}}, \boldsymbol{\theta}\right) E S_{\alpha}^{\prime}\right)+\left(1-p_{0}\right)\left(\left(E S_{\alpha}^{\prime}\right)^{T} M^{-1}\left(\xi_{\widehat{\boldsymbol{\theta}}}, \boldsymbol{\theta}\right) E S_{\alpha}^{\prime}\right)}\right)$ where $p_{0}=\sqrt{n} / n$. 


\section{A.15 A New Multivariate Exponential Probability Density}

$f\left(t_{1}, t_{2}, \ldots, t_{R}\right)$

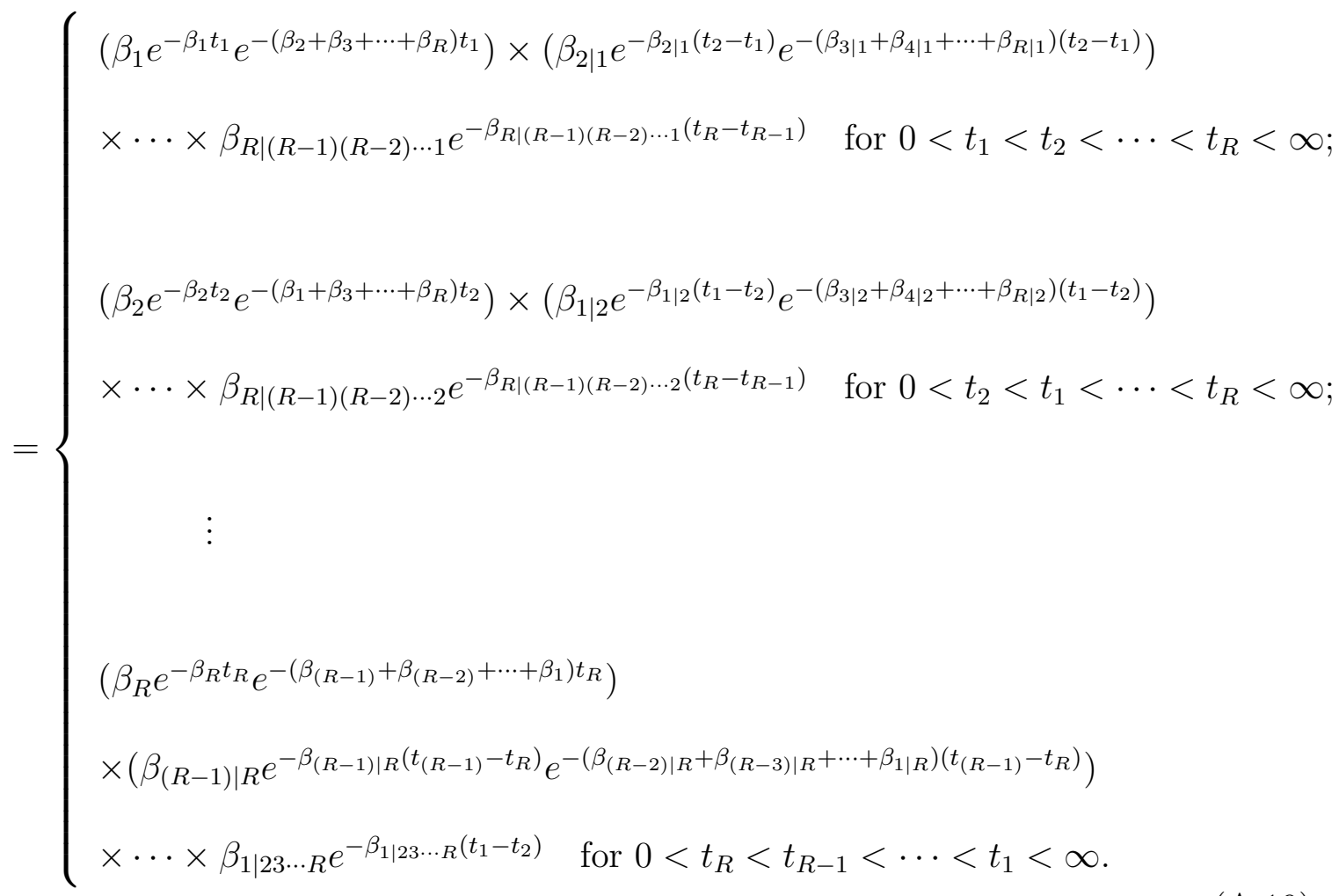




\section{A.16 A New Multivariate Weibull Probability Den- sity}

$f\left(y_{1}, y_{2}, \ldots, y_{R}\right)$

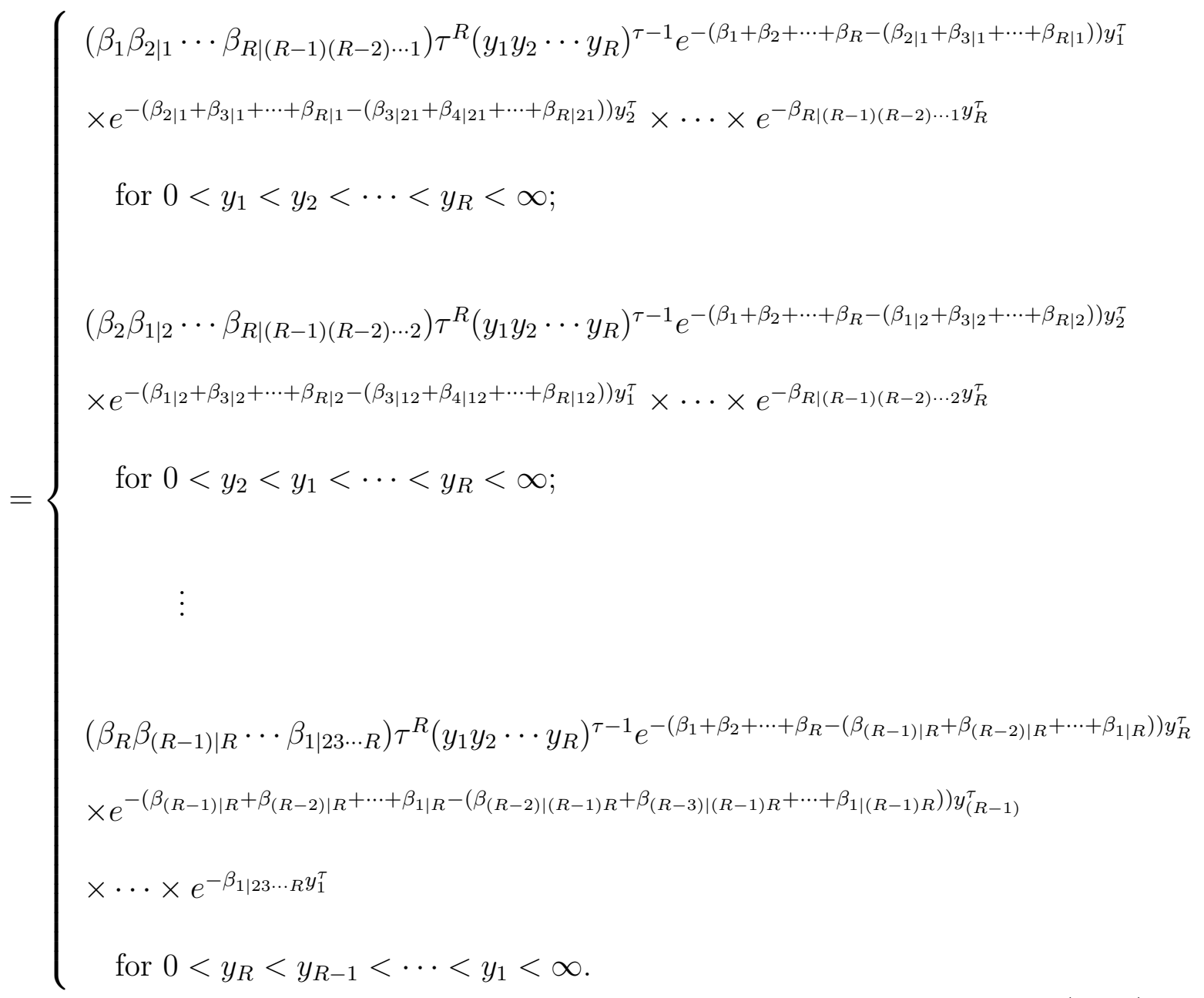




\section{Bibliography}

[1] Atkinson, A.C., Donev, A.N., Tobias, R.D. (2007). Optimum Experimental Designs, with SAS. Oxford Statistical Science Series 34.

[2] Balakrishnan, N., Lai, C.D. (2009). Continuous bivariate distribution. 2nd edition. Springer

[3] Box, G., Hunter, W. (1965). Sequential design of experiments for nonlinear models. Proceedings IBM Scientific Computing Symposium Statistics, 113-137.

[4] Cook, D., Fedorov, V. (1995). Constrained optimization of experimental design. Statistics 26, 129-178.

[5] Dette, H., Bornkamp, B., Bretz, F. (2013). On the efficiency of two-stage response-adaptive designs. Statistics in Medicine 32, 1646-1660.

[6] Dragalin, V, Fedorov, V.V (2006). Adaptive designs for dose-finding based on efficacy-toxicity response. Journal of Statistical Planning and Inference 136, $1800-1823$.

[7] Dragalin, V, Fedorov, V.V, and Wu, Y. (2006). Optimal designs for bivariate probit model. GlaxoSmithKline Pharmaceuticals Technical Report 2006-01.

[9] Dragalin, V, Fedorov, V.V, and Wu, Y. (2008). Adaptive designs for selecting drug combinations based on efficacy-toxicity response. Journal of Statistical Planning and Inference 138, 352-373.

[9] Dragalin, V, Fedorov, V.V, and Wu, Y. (2008). Two-stage design for does-finding that accounts for both efficacy and safety. Statistics in Medicine 27, 5156-5176.

[10] Durante, F. (2007). A new class of bivariate copulas. Comptes Rendus Mathematique 344, 195-198. 
[11] Fedorov, V.V. (1972). Theory of Optimal Experiments. Academic Press: New York.

[12] Fedorov, V.V, and Hackl, P. (1997). Model-Oriented Design of Experiments. Lecture Notes in Statistics. 125. New York: Springer-Verlag.

[13] Fedorov, V.V, and Wu, Y. (2007). Does finding designs for continuous responses and binary utility. Journal of Biopharmaceutical Statistics 17, 1085-1096.

[14] Ferguson, T.S (1996). A Course in Large Sample Theory. Chapman \& Hall/CRC.

[15] Ford, I., and Silvey, S.D.(1980). A sequentially constructed design for estimating a nonlinear parametric function. Biometrika 67, 381-388.

[16] Fréchet, M. (1951). Sur les tableaux de corrélation dont les marges sont données. Ann. Univ. Lyon Sect. A, Series 3 14, 53-77.

[17] Frees, E.W., Valdez, E.A. (1998). Understanding relationship using copulas. North-America Actuarial Journal 2, 1-26.

[18] Freund, J.E. (1961). A bivariate extension of the exponential distribution. Journal of the American Statistical Association 56, 971-977.

[19] Fuller, D., Scherer W. (1998). The desirability function: underlying assumptions and aplication implications. IEEE, 4016-4021.

[20] Gaivoronski, A., Fedorov, V. (1984). Design of experiments under constraints. International Institute for Applied Systems Analysis, WP-84-8, Laxenburg, Austria.

[21] Genest, C., MacKay, J (1986). Copules archimédiennes et familles de lois bidimensionnelles dont les marges sont données. Canadian Journal of Statistics 14, $145-159$.

[22] Gumbel, E.J. (1960). Bivariate exponential distribution. Journal of the American Statistical Association 55, 698-707.

[23] Haines, L.M, Perevozskaya, I., Rosenberger, W.F. (2003). Bayesian optimal designs for Phase I clinical trials. Biometrics 59, 591-600.

[24] Hanagal, D.D. (2005). A bivariate Weibull regression model. Economic Quality Control 20, 1-8.

[25] Hoeffding, W. (1940). Scale-invariant correlation theory. The Collected Works of Wassily Hoeffding, Springer-Verlag, 57-107. 
[26] Klein, J.K, Moeschberger, M.L. (2003). Survival Analysis. 2nd Edition. Springer, pp.39.

[27] Kiefer, J. (1974). General equivalence theory for optimum designs. The Annals of Statistics 2, 849-879.

[28] Kiefer, J., Wolfowitz, J. (1960). The equivalence of two extremum problems. Canadian Journal of Mathematics 12, 363-366.

[29] Lane A., Yao, P., and Flournoy, N. (2014). Information in two-stage adaptive optimal design. Journal of Statistical Planning and Inference, 181-194.

[30] Marshall, A.W and Olkin, I. (1967). A multivariate exponential distribution. Journal of the American Statistical Association 62, 30-44.

[31] Marshall, A.W., Olkin, I. (1988). Families of multivariate distributions. Journal of the American Statistical Association 83, 834-841.

[32] Morgenstern, D. (1956). Einfache Beispiele Zweidimensionaler Verteilungen. Mitteilingsblatt fur Mathematische Statistik 8, 234-235.

[33] Nelsen, R.B. (2006). An Introduction to Copulas. 2nd edition. Springer-Verlag, New York, pp.110.

[34] Pronzato, L., Pázman, A. (2013). Design of Experiments in Nonlinear Models. Lecture Notes in Statistics. 212. Springer.

[35] Rüschendorf, L. (1985). Construction of multivariate distributions with given marginals. Annals of the Institute of Statistical Mathematics 37, 225-233.

[36] Sklar, A. (1959). Fonctions de répartition á n dimensions et leurs marges. Publ. Inst. Statist. Univ. Paris 8, 229-231.

[37] White, L. (1973). An extension of the general equivalence theorem to nonlinear models. Biometrika 60, 345-348.

[38] White, L. (1975). The optimal design of experiments for estimation of nonlinear models. Dissertation. University of London, UK.

[39] Wolfram Research, Inc. (2012). Mathematica (Version 9.0). Wolfram Research, Inc., Champaign, Illinois. 


\section{VITA}

Sungwook Kim was born on December 25, 1980 in Seoul, South Korea. He graduated from Sejong University in 2005 and received his Bachelor Degree in Mathematics in 2005. He received his Master Degree in Mathematics in 2007 at Pittsburg State University. He joined the Department of Statistics at the University of Missouri in August 2008, and he worked on his dissertation under the direction of his advisor Dr. Nancy Flournoy. He will receive his Ph.D. in Statistics in Spring 2014. 